\title{
Analyzing dispersion properties of hole and ring like photonic crystal waveguides by introducing systematic shift and twist
}

\section{Vadapalli Durga Rama Pavan}

National Institute of Technology Warangal

Sourabh Roy ( $\sim$ sroy@nitw.ac.in )

National Institute of Technology Warangal https://orcid.org/0000-0002-8034-7367

\section{Research Article}

Keywords: Dispersion engineering, slow light, optical buffer, geometrical engineering, wideband waveguides

Posted Date: April 6th, 2021

DOI: https://doi.org/10.21203/rs.3.rs-223506/v1

License: (c) (i) This work is licensed under a Creative Commons Attribution 4.0 International License.

Read Full License 


\title{
Analyzing dispersion properties of hole and ring like photonic
} crystal waveguides by introducing systematic shift and twist

\author{
Vadapalli Durga Rama Pavan and Sourabh Roy* \\ Department of Physics, National Institute of Technology, Warangal - 506004 (India)
}

*sroy@nitw.ac.in

\begin{abstract}
:
Dispersion engineering of photonic crystal waveguides is attractive due to their potential applications in linear and nonlinear phenomena. Here, we present a comprehensive and systematic study to achieve the increased control over the dispersion curve of the waveguide, operating at telecom wavelengths. The effect of the radius of air cylinders, and their lattice position on the dispersion features is studied chiefly in a line-defect photonic crystal waveguide. For this purpose, perturbations were introduced in the radius and position of the air cylinders. With the help of MIT Photonic Bands software, group index and dispersion coefficients were calculated to characterize the features of the waveguide. Ring like structures were introduced in the innermost rows to increase the impact to further level. With this systematic study, one can tune the waveguide with desired range of group index and bandwidth with controlled dispersion properties. Present study resulted with the flat group index in the range of 31.42 to 7.64 over a wavelength range of $7.97 \mathrm{~nm}$ to $30.41 \mathrm{~nm}$ with very low dispersion. The developed structures may find applications in optical delays, optical buffers and nonlinear applications.
\end{abstract}

Keywords: Dispersion engineering, slow light, optical buffer, geometrical engineering, wideband waveguides.

\section{Introduction:}

Dispersion engineering of a photonic crystal waveguide (PCW) is interesting because of its capability in slow light (SL) generation, nonlinear effects, optical buffers and optical delays (Schulz et al. 2010; Baba 2008; Krauss 2007). In specific, light modes in PCW exhibit smaller group velocities near the Brillouin zone edge (Letartre et al. 2001; Vlasov et al. 2005). At these lower group velocities, exploring the PCW can provide enhanced light matter interactions and nonlinear effects (Schulz et al. 2010; Vlasov et al. 2005; (Soljačić and Joannopoulos 2004; Li and Zhou 2006).

In the past two decades scientific community has shown greater interest in the tailoring of PCW for nonlinear applications (Colman et al. 2012; Ebnali-Heidari et al. 2009; Hou et al. 2009; Roy et al. 2012; Roy et al. 2012). For dispersion engineering, the geometrical parameters and the effective refractive index of the PCW were modified to achieve various 
applications. By changing the diameter of the air cylinders (Frandsen et al. 2006) PCW with controlled group velocity and semi-slow light is reported. This change in diameter has achieved various features such as group velocity in the range of $\sim \mathrm{c}_{\mathrm{o}} / 100$, low propagation loss, and controlled group velocity dispersion (GVD). Varying the waveguide width has produced control over the group velocity and this method resulted with constant group velocity and even zero third order dispersion (Petrov and Eich 2004). The position of the air cylinder is shifted systematically to design PCW with flat band slow light and reported nonlinear enhancement ( $\mathrm{Li}$ et al. 2008; Hamachi et al. 2009). High and nearly constant group indices were presented in these cases.

In some studies, researchers have introduced a ring like structure in the PCW (Hou et al. 2009; Säynätjoki et al. 2007) to minimize the dispersion. A flat band line defect PCW is developed by introducing these rings in the lattice and very low GVD was achieved. Dispersion engineering was also reported by filling of selective micro-fluids in the lattice of PCW (Ebnali-Heidari et al. 2009; Casas-Bedoya et al. 2012). By introducing the micro-fluids in the lattice, group velocities of the range of $\sim \mathrm{c}_{\mathrm{o}} / 110$ were attained. Also, this mechanism helped to design reconfigurable PCW. All these studies aim to control the dispersion, achieving high and constant group indices, and enhancement of nonlinear properties in the PCW, to find the applications in optical delays, nonlinear effects, all optical circuits and onchip devices.

In this article, we revisit the dispersion engineering in a systematic way by studying the effect of radius of air cylinders and their lattice position on the linear and nonlinear properties of the line-defect waveguide. The study aims to present the combined effect of the geometrical perturbations on the dispersion features of line-defect PCW. Initially, the impact of change in radius and lattice position is studied independently. Radius of the air cylinders was optimized between $0.21 a$ and $0.30 a$ and lattice perturbations in the range of $0.00 a$ to $0.20 a$ were used for this study. At the latter stage, the combined effect of all these geometrical variations was incorporated. Group index $\left(n_{g}\right)$, second order dispersion $\left(\beta_{2}\right)$ and fourth order dispersion $\left(\beta_{4}\right)$ were studied principally for the description of the nonlinear parameters. Ring like structures were introduced in the first and second innermost rows of the waveguide to produce further impact. With this rigorous study, we noticed that the combined shift and twist effects with optimized radius of air cylinders resulted higher and flat group index with considerably larger bandwidth. By this method, we propose an organized way to develop the PCWs with desired delay and wide ranges of bandwidth. These PCWs can be used as optical buffers, optical switches, and to produce nonlinear effects such as parametric amplification. 


\section{Geometrical Design:}

Figure 1 (a) shows the geometrical design of the PCW structure. A 2-D periodic hexagonal air-cylinder pattern with lattice constant $(a) 465 \mathrm{~nm}$ is used as the base structure. Air cylinders with radius $0.25 a(116.25 \mathrm{~nm})$ in GaAs substrate is used for this purpose. One row of air cylinders (row-8) is eliminated to form the waveguide (W15) structure with waveguide width $1.72 a(800 \mathrm{~nm})$. Figure 1 (b) shows the partial filling of air holes with the same material to form a ring-like structure (rings). The holes, just adjacent to the waveguide, up to second row were filled to form the rings. The inner cylinder's radius $\left(\mathrm{r}^{\prime}\right)$ is calculated as $0.12 a(55.8 \mathrm{~nm})$. Figure 1 (c) shows the proposed study in a nutshell. To investigate the properties of PCW, in the commencement of the work, we studied the effect of radius of holes, next to the waveguide. Latter studies involved the lattice shift and twist of the holes. Also, in the ring like structure, the upshot of change in radius, shift and twist are examined. Besides, we studied the integrated effect of radius, shift and twist on the dispersion features of the PCW.

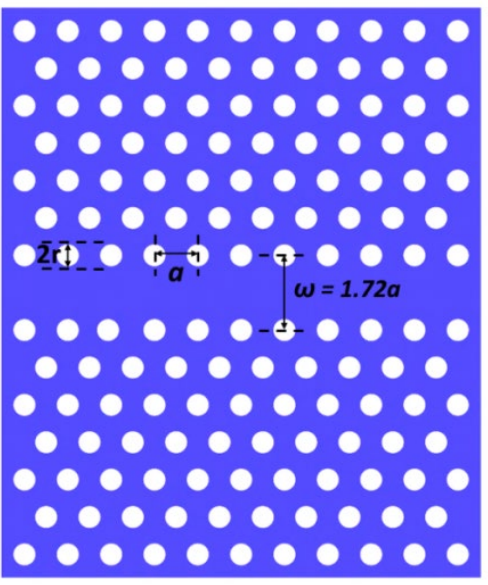

(a)

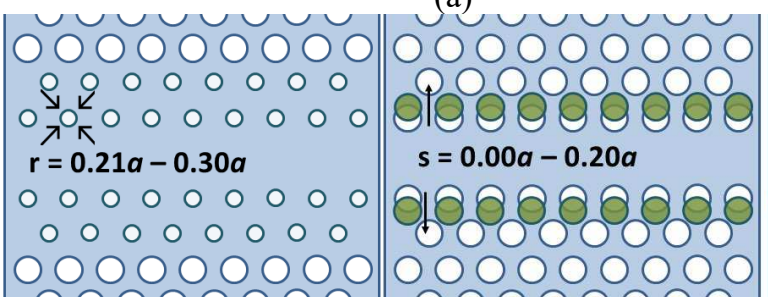

(c)

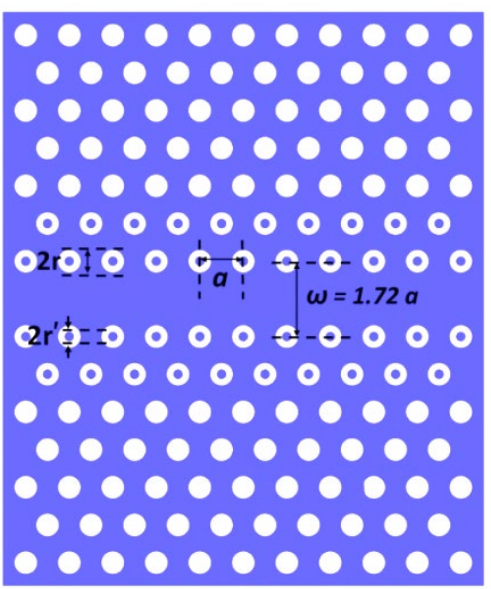

(b)

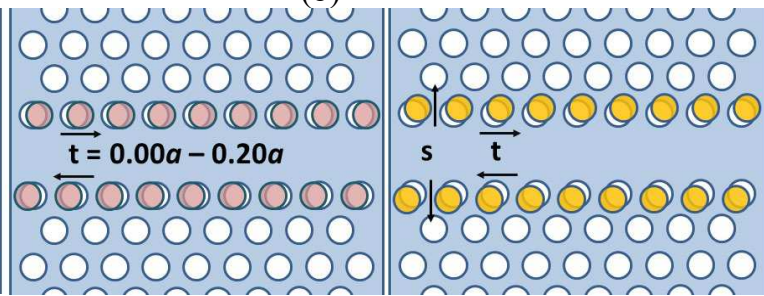

(c)

Fig. 1 Schematic of the structure. (a) 2-D hexagonal lattice with air cylinders to form the waveguide. (b) waveguide with rings (c) illustration of change in radius, introducing shift and twist effects.

\section{Analyzing parameters:}

In this study, MIT Photonic Bands (MPB) (Johnson 2001) on Ubuntu platform was used to simulate the structure and to calculate the group velocity and group index values at a given frequency. The group index which is considered as the slow-down factor or the delay is 
defined as $n_{g}=\left(c / v_{g}\right)$. One important parameter in dispersion engineered PCW is the group velocity $v_{g}$, defined as the inverse of the first order dispersion (Baba 2008)

$$
v_{g}=\left(\frac{d k}{d \omega}\right)^{-1}
$$

where $k$ is the wave-number and $\omega$ is the angular frequency. The other parameter which determines the nonlinear behaviour of the light in PCW is the $\beta_{2}$ or GVD, which is defined as (Hou et al. 2009)

$$
\beta_{2}=\left(\frac{d^{2} k}{d \omega^{2}}\right)
$$

The PCW is best described with $n_{g}, \mathrm{GVD}$ or $\beta_{2}$ and the normalized bandwidth. However, for a given structure, the $n_{g}$ and the bandwidth over which $n_{g}$ is maintained constant are inversely proportional and hence to quantify the performance of the PCW, normalized delay-bandwidth product (NDBP) is defined (Schulz et al. 2010), which is the product of average group index $n_{g}$ and normalized bandwidth $\left(\Delta \omega / \omega_{o}\right)$

$$
N D B P=n_{g} \cdot\left(\Delta \omega / \omega_{o}\right)
$$

Hence, for applications like SL, one requires the PCW with large DBP, so that it provides larger buffering time. In this study, for every structure we present the average $n_{g}$, bandwidth, $\beta_{2}$ and $\beta_{4}$ parameters and their changes with geometrical perturbations.

\section{Results and Discussion:}

Figure 2 shows the photonic band diagram of the base structure. The substrate band exists between band 14 and 15. The first photonic bandgap (PBG) exists between band 15 and 16 . Theoretically it is calculated and found to be $0.006215 \mathrm{c} / \mathrm{a}$. Using the lattice constant's value, it is found to be $28.5062 \mathrm{~nm}$.

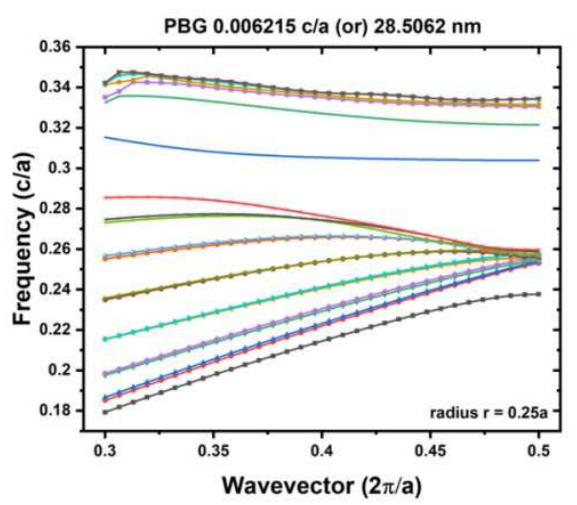

Fig. 2 Photonic band diagram of the structure. The PCW structure with hole radius $r=0.25 a$ is taken as a reference in the study. 


\subsection{Effect of radius:}

In this step, the radius of the air cylinders and the rings was optimized to achieve the control over the dispersion curve. Further, these achieved results were used in next steps to increase the dispersion features. As discussed by (Frandsen et al. 2006; Petrov and Eich 2004) the change in radius of the air cylinder provides a great control over the group velocity. To follow-up this study, at first, the effect of radius of hole on the PBG and the dispersion properties is studied. The line defect in PCW supports the modes that are categorized as index guided and gap guided (Notomi et al. 2001). The interactions between these modes determine the realization of nonlinear effects in the PCW. To study the effect of radius of hole on the PBG of the structure, band 15 is considered for further analysis. In the simulation, the radius of the air hole, just adjacent to the waveguide is changed and the corresponding bands are plotted with wave vector. In the figure 3 (a) band 15 is plotted with wave vector for PCW with air cylinders. Different curves in the plot represent the band 15 (band edge) of the structure with different hole radius. Figure 3 (b) represents the same of the PCW with rings. In the rings structure, only the radius of outer cylinder is changed from $0.21 a$ to $0.30 a$ while the inner cylinder's radius remains kept constant as $0.12 a$. The slope of these dispersion curves gives the group velocity of the mode. The increase in the slope of these dispersion curves with decrease in the radius of hole indicates the possibility of flat band region. This flat band is an indication of the possibility of achieving nonlinear effects such as four wave mixing (FWM), parametric amplification (PA), supercontinuum generation (SCG) and SL. Therefore, nonlinear effects could be generated in this region with decreasing the radius of the holes adjacent to the waveguide and by introducing the rings in the structure.

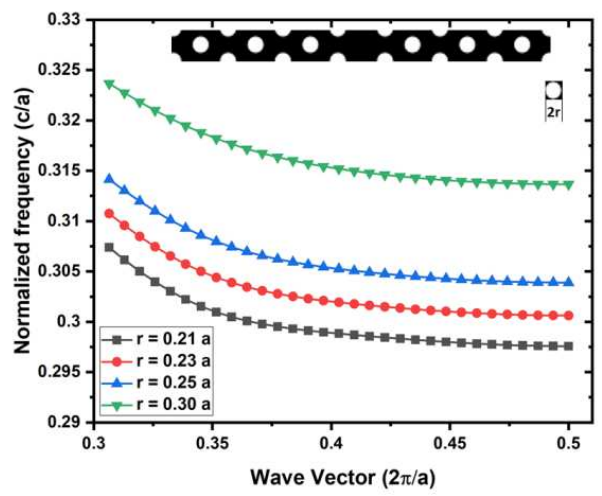

(a)

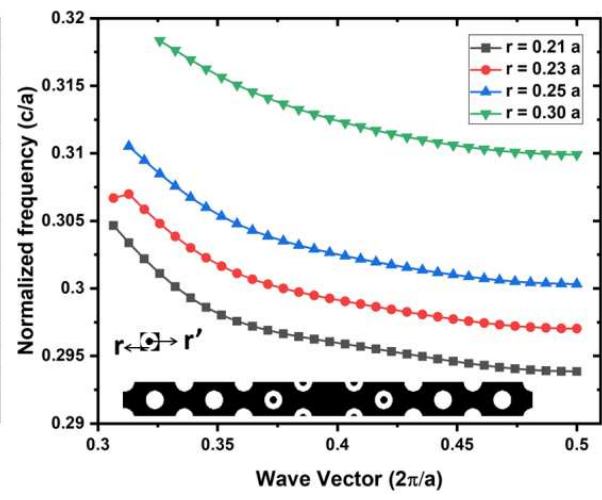

(b) 


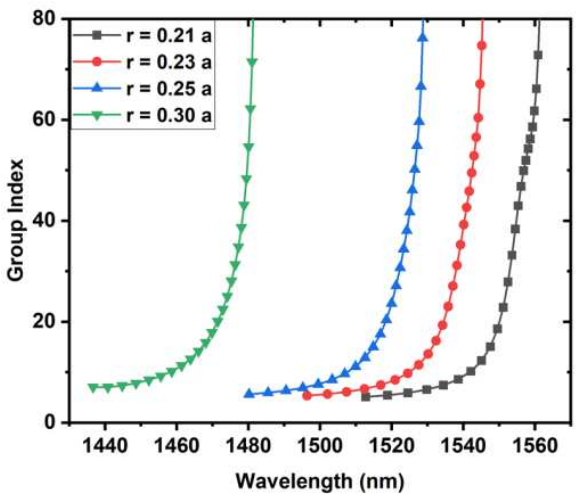

(c)

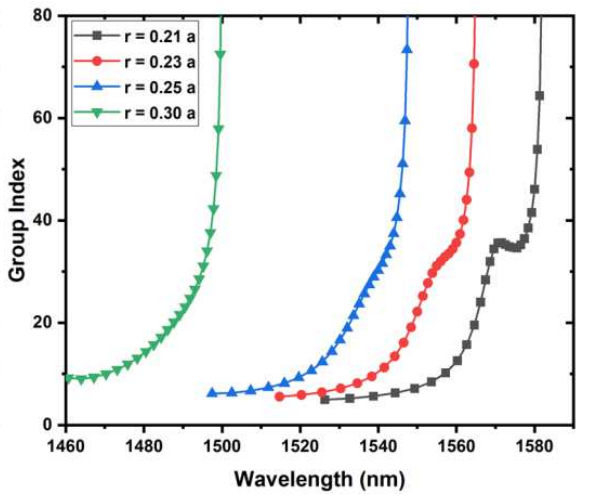

(d)

Fig. 3 Band 15 from the band diagram of the PCW (a) with holes and (b) with rings. Group index plot of the PCW (c) with holes and (d) with rings.

For studying the features of the PCW, the calculated $n_{g}$ is plotted against the calculated wavelengths (figure 3 (c-d)). As discussed by others (Bagci and Akaoglu 2012) the wavelength and $n_{g}$ plot changes from shoulder to step-like shape in case of holes and to Ushape in case of rings. In both the cases, $\mathrm{r} / a=0.21$ resulted with possibility of nonlinear effects. Variation of $\beta_{2}$ and $\beta_{4}$ (fourth order dispersion) with wavelength were shown in the figure $4(\mathrm{a}-\mathrm{d})$ for the structures with holes and rings respectively. At $\mathrm{r} / a=0.21$, the smallest among our considered fraction filling values, $\beta_{2}$ and $\beta_{4}$ have a clear change from positive to negative range and vice versa. This reveals the possibility of dispersion compensation in the structure. In contrast to this, the increase in the radius of the hole and ring has lowered the changes in these values which confirm the possible distortion of optical pulse in the PCW.

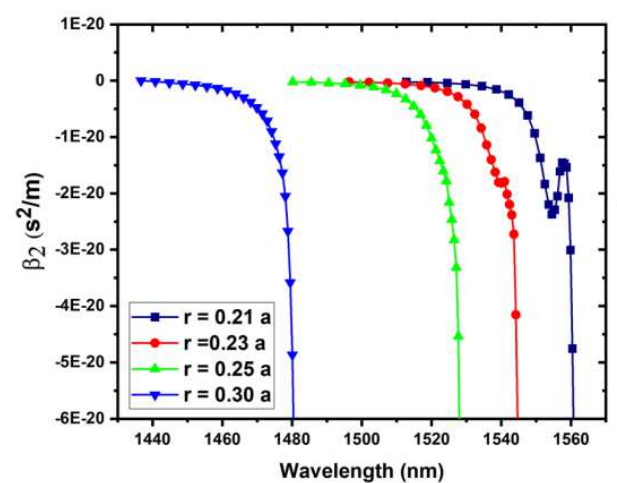

(a)

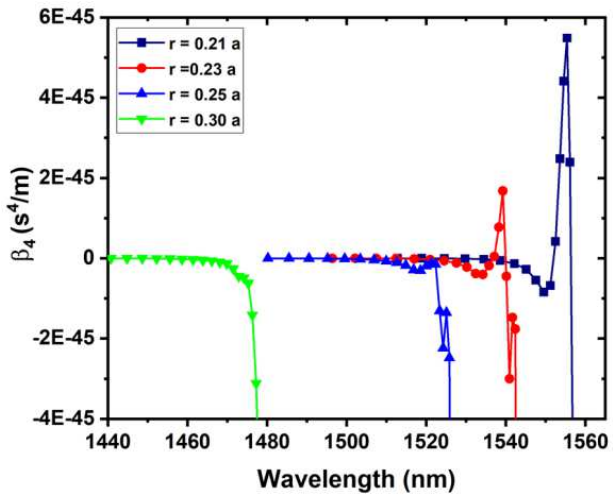

(b) 


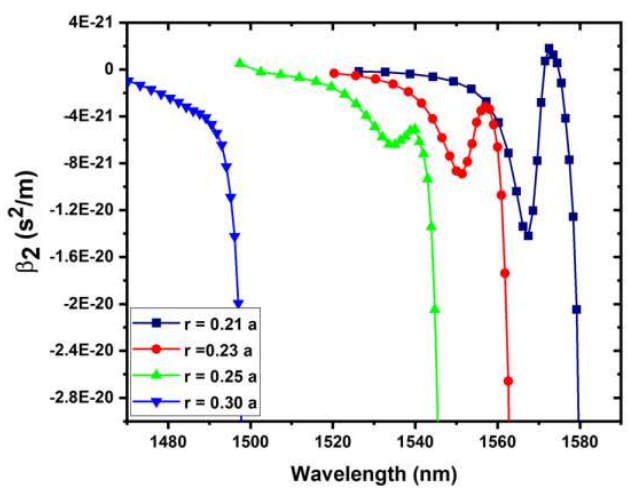

(c)

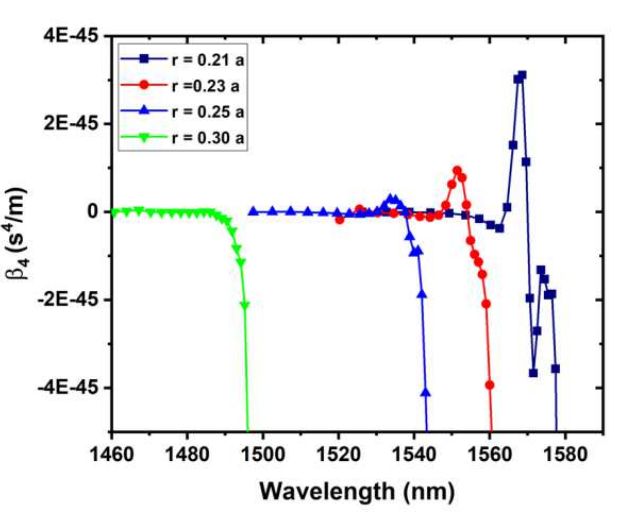

(d)

Fig. 4 Dispersion characteristics corresponding to figure 3 (c-d). (a) $\beta_{2}$ of PCW with holes (b) $\beta_{4}$ of PCW with holes (c) $\beta_{2}$ of PCW with rings (d) $\beta_{4}$ of PCW with rings.

Here, in the initial structure with $\mathrm{r}=0.25 a$ no nonlinear effects were observed. However, the change in radius of hole affects the profiles of $\beta_{2}$ and $\beta_{4}$. At $\mathrm{r}=0.21 a$, considerable changes in $n_{g}, \beta_{2}$ and $\beta_{4}$ were observed. Though technologically alteration of the hole size is challenging, yet it is interesting due to the nonlinear applications. In the further study, this optimized radius value $r=0.21 a$ is used to improve the features of the structure.

\subsection{Effect of lattice shift:}

By changing the position of the holes in the PCW, one can produce a nearly constant group index over a large bandwidth ( $\mathrm{Li}$ et al. 2008). The shift in the lattice produces different linear and nonlinear effects, which have potential applications (Hamachi et al. 2009; Tang et al. 2013; Yang et al. 2011). The shift introduced in the lattice directly impacts the dispersive curve and produces flat band. Considering the previously published results, the shift (s) in the lattice is introduced in the range of $0.00 a-0.20 a$ with $0.04 a$ step. From our first result, the radius of the holes and rings remains taken as $0.21 a$. So far, either the position or the radius of the holes and rings was changed to enhance the features of PCW. In this study, we combine both the effects and calculated the resultant performance of the PCW. The position of the band 15 is plotted against the wave vector and the first and second order derivatives were calculated. Table 1 gives the summary of $n_{g}$ and bandwidth with the shift in lattice in holes and rings structure. 


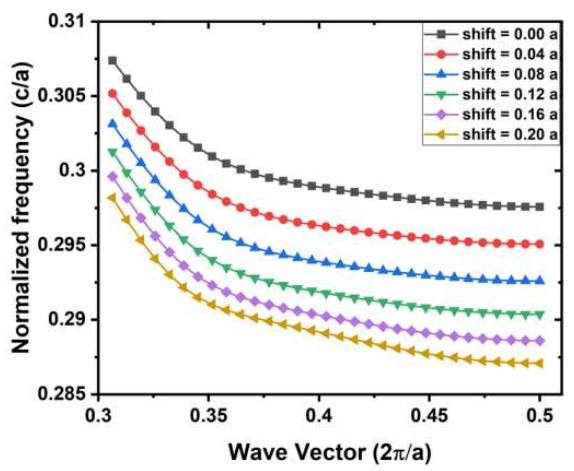

(a)

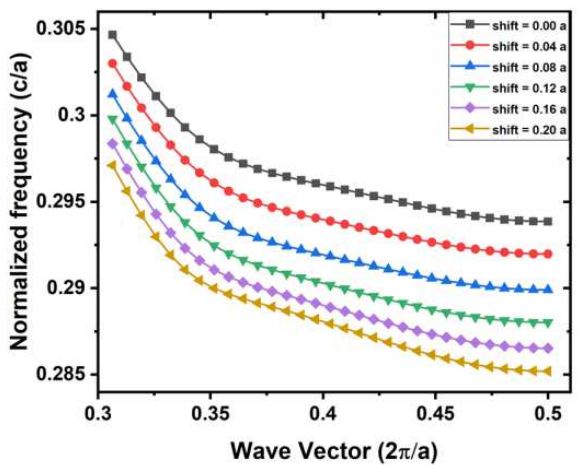

(c)

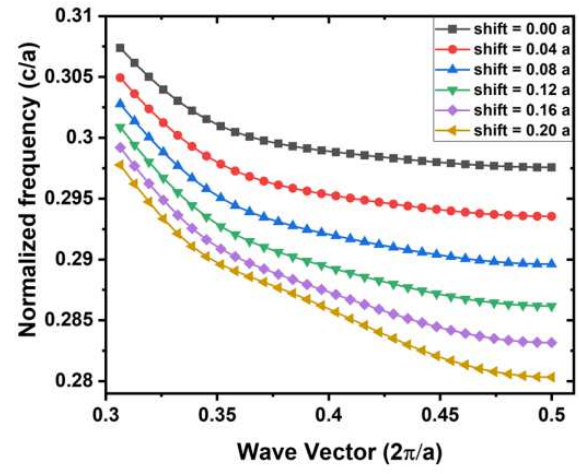

(b)

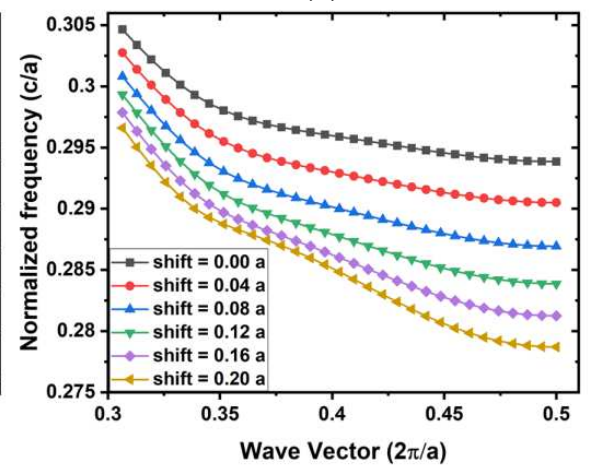

(d)

Fig. 5 Band 15 from the band diagram of PCW with holes and (a) shift in one row (b) shift in two rows. Band 15 from the band diagram of PCW with rings and (c) shift in one row (d) shift in two rows.

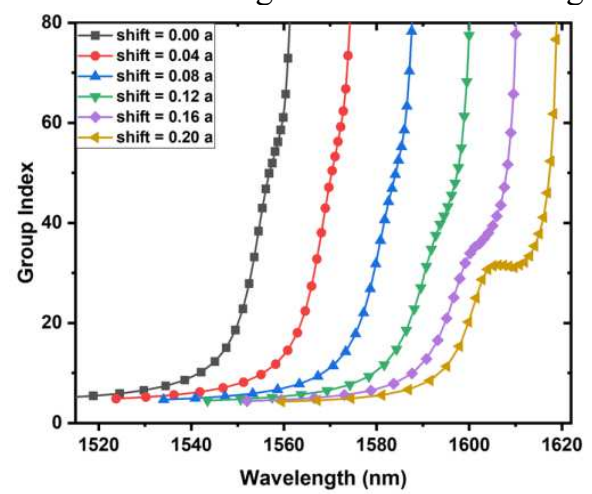

(a)

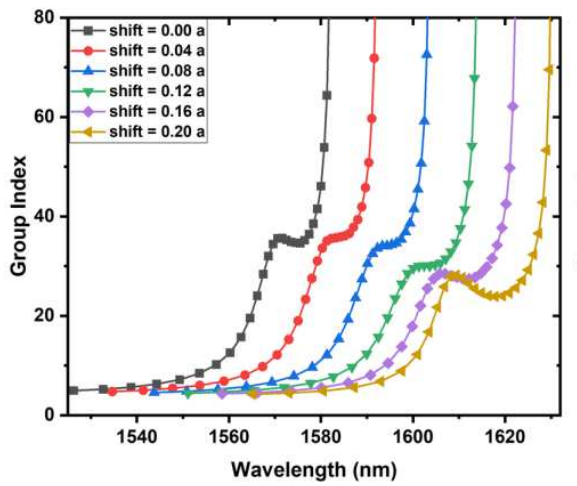

(c)

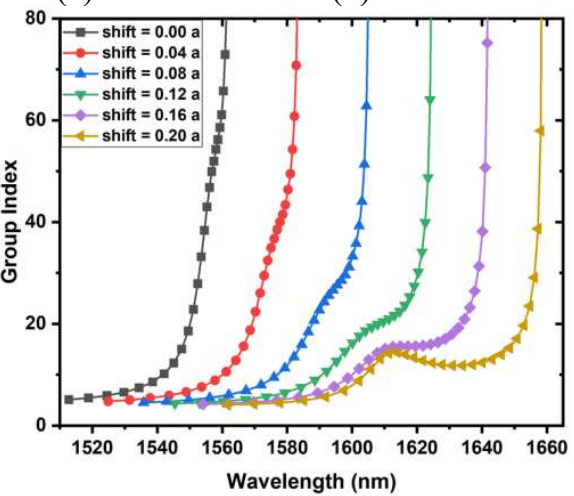

(b)

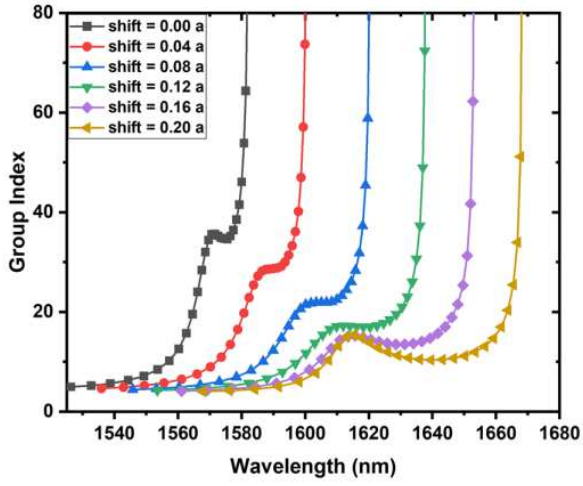

(d)

Fig. 6 Group index - wavelength plot of the PCW with holes and (a) shift in one row (b) shift in two rows. Group index - wavelength plot of the PCW with rings and (c) shift in one row (d) shift in two rows. 
Table 1: Calculated group index and bandwidth of the waveguide at different shift values in first and second rows

\begin{tabular}{|l|l|l|l|l|l|}
\hline \multicolumn{3}{|c|}{ Holes } & \multicolumn{3}{c|}{ Rings } \\
\hline shift (s) & \multicolumn{1}{|c|}{$n_{\boldsymbol{g}}$} & $\begin{array}{c}\text { bandwidth } \\
(\mathbf{n m})\end{array}$ & shift (s) & $\boldsymbol{n}_{\boldsymbol{g}}$ & $\begin{array}{c}\text { bandwidth } \\
(\mathbf{n m})\end{array}$ \\
\hline \multicolumn{7}{|c|}{ Shift in one row } \\
\hline $0.00 a^{*}$ & - & - & $0.00 a$ & 34.72 & 1.98 \\
\hline $0.04 a^{*}$ & - & - & $0.04 a$ & 35.55 & 2.92 \\
\hline $0.08 a^{*}$ & - & - & $0.08 a$ & 34.27 & 3.09 \\
\hline $0.12 a^{*}$ & - & - & $\mathbf{0 . 1 2 a}$ & $\mathbf{3 0 . 4 1}$ & $\mathbf{5 . 8 7}$ \\
\hline $0.16 a^{*}$ & - & - & $0.16 a$ & 27.58 & 3.92 \\
\hline $0.20 a$ & $\mathbf{3 1 . 4 2}$ & $\mathbf{7 . 9 7}$ & $0.20 a$ & 24.22 & 6.01 \\
\hline \multicolumn{7}{|c|}{ Shift in two rows } \\
\hline $0.00 a^{*}$ & - & - & $0.00 a$ & 34.72 & 1.98 \\
\hline $0.04 a^{*}$ & - & - & $0.04 a$ & 28.66 & 6.1 \\
\hline $0.08 a^{*}$ & - & - & $0.08 a$ & 21.82 & 8.16 \\
\hline $0.12 a$ & 20.70 & 1.74 & $\mathbf{0 . 1 2 a}$ & $\mathbf{1 7 . 1 7}$ & $\mathbf{1 4 . 8}$ \\
\hline $0.16 a$ & $\mathbf{1 5 . 5 6}$ & $\mathbf{1 3 . 9 5}$ & $0.16 a$ & 13.73 & 13.49 \\
\hline $0.20 a$ & 11.88 & 9.36 & $0.20 a$ & 10.54 & 14.22 \\
\hline
\end{tabular}

*No flat band is observed with constant group index.

Out of all these structures, PCW rings structure with fraction filling ratio $\mathrm{r} / a=0.21$ and shift $=0.12 a$ resulted with highest DBP.

Like in the previous case, $\beta_{2}$ and $\beta_{4}$ were calculated and plotted against the wavelength. Here the shift is applied in first and second rows of the holes and rings of the structure to enhance the features of PCW. As the shift in lattice is increased, a gradual increase in the 'band' of $\beta_{2}$ and $\beta_{4}$ curves is observed. In the figures $7 \& 8$, we have shown the selective plots of $\beta_{2}$ and $\beta_{4}$ against wavelength respectively. The table 2 gives the introduced shift, corresponding maximum and minimum values of $\beta_{2}$ and $\beta_{4}$ for both holes and rings structures.

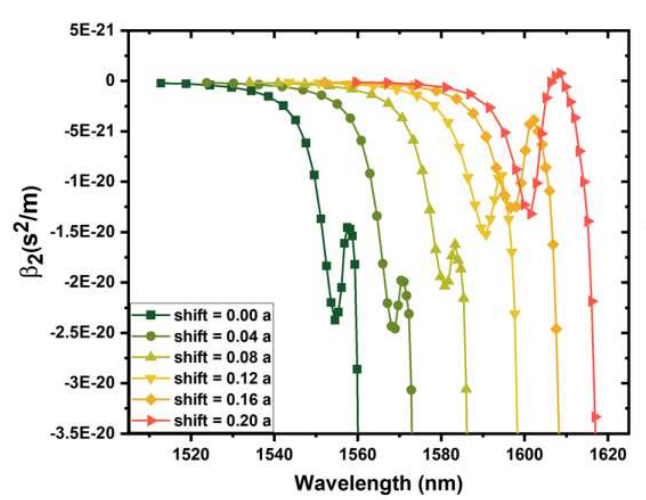

(a)

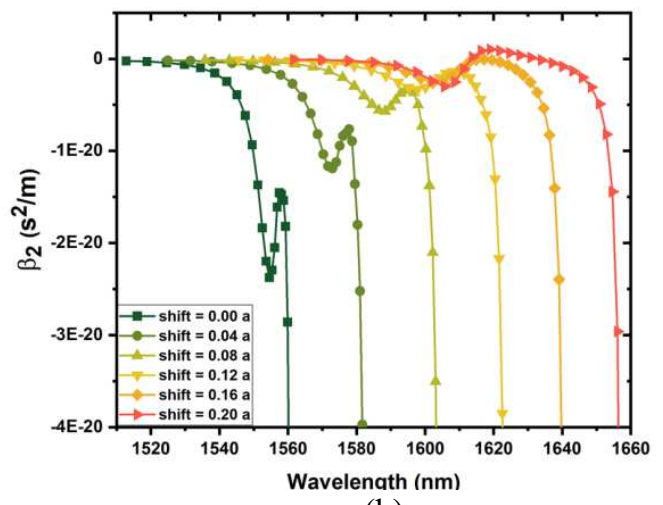

(b) 


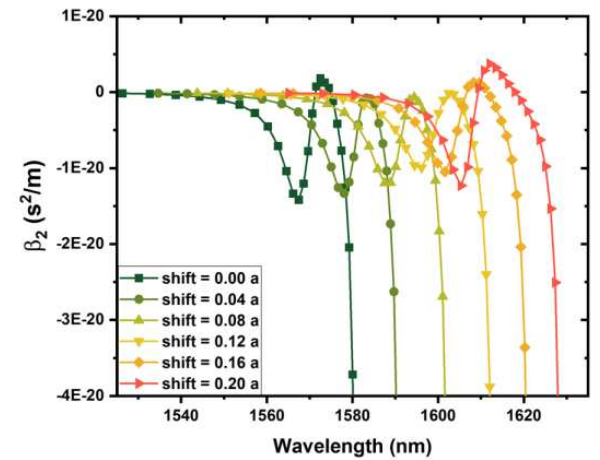

(c)

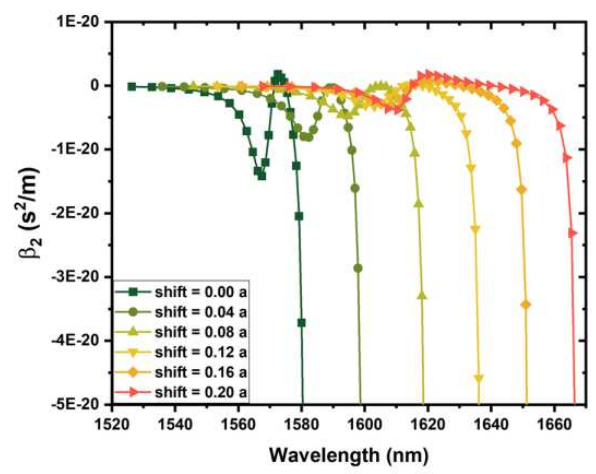

(d)

Fig. 7 Dispersion characteristics corresponding to figure 6 (a-d). (a) $\beta_{2}$ of PCW with holes and shift in one row (b) $\beta_{2}$ of PCW with holes and shift in two rows (c) $\beta_{2}$ of PCW with rings and shift in one row (d) $\beta_{2}$ of PCW with rings and shift in two rows.

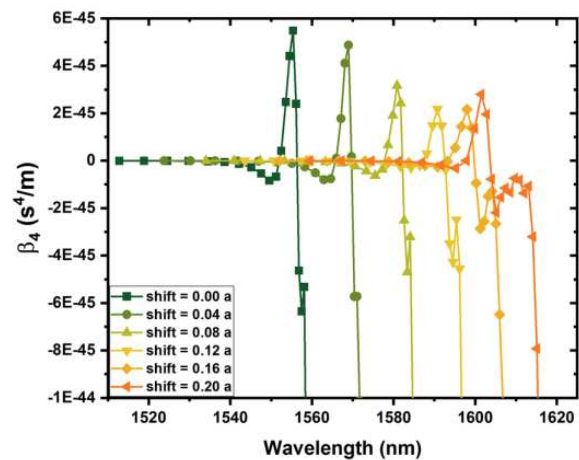

(a)

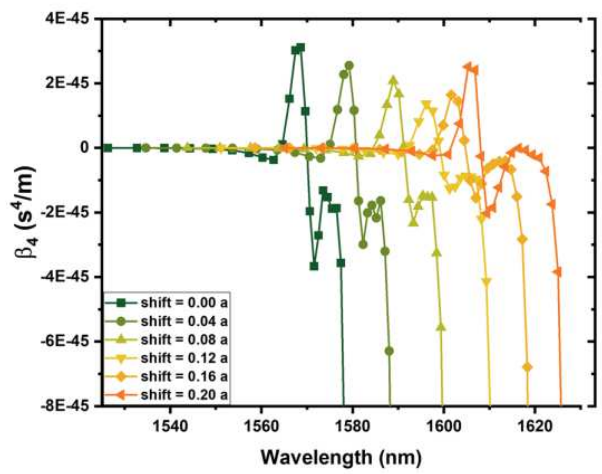

(c)

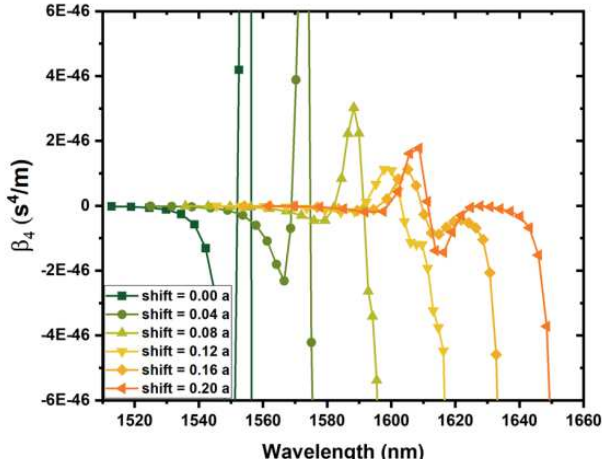

(b)

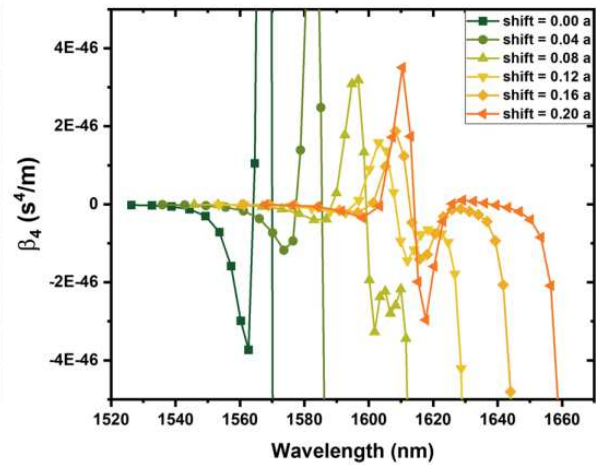

(d)

Fig. 8 Dispersion characteristics corresponding to figure 6 (a-d). (a) $\beta_{4}$ of PCW with holes and shift in one row (b) $\beta_{4}$ of PCW with holes and shift in two rows (c) $\beta_{4}$ of PCW with rings and shift in one row (d) $\beta_{4}$ of PCW with rings and shift in two rows.

Table 2: Calculated $\beta_{2}$ and $\beta_{4}$ values of the waveguide with applied shift.

\begin{tabular}{|c|c|c|c|c|c|c|c|c|c|}
\hline \multicolumn{5}{|c|}{ Holes } & \multicolumn{5}{|c|}{ Rings } \\
\hline $\begin{array}{l}\text { shift } \\
\text { (s) }\end{array}$ & $\begin{array}{c}\boldsymbol{\beta}_{2}(\max .) \\
\times 10^{-20} \\
\mathbf{s}^{2} / \mathbf{m}\end{array}$ & $\begin{array}{c}\boldsymbol{\beta}_{2}(\min .) \\
\times 10^{-20} \\
\mathbf{s}^{2} / \mathbf{m}\end{array}$ & $\begin{array}{c}\beta_{4}(\max .) \\
\times 10^{-45} \\
\mathbf{s}^{4} / \mathbf{m}\end{array}$ & $\begin{array}{c}\beta_{4}(\min .) \\
\times 10^{-45} \\
\mathbf{s}^{4} / \mathbf{m}\end{array}$ & $\begin{array}{l}\text { shift } \\
\text { (s) }\end{array}$ & $\begin{array}{c}\boldsymbol{\beta}_{2}(\max .) \\
\times 10^{-20} \\
\mathbf{s}^{2} / \mathbf{m}\end{array}$ & $\begin{array}{c}\beta_{2}(\min .) \\
\times 10^{-20} \\
\mathrm{~s}^{2} / \mathrm{m}\end{array}$ & $\begin{array}{c}\beta_{4}(\max .) \\
\times 10^{-45} \\
\mathbf{s}^{4} / \mathrm{m}\end{array}$ & $\begin{array}{c}\beta_{4}(\min .) \\
\times 10^{-45} \\
\mathbf{s}^{4} / \mathbf{m}\end{array}$ \\
\hline \multicolumn{10}{|c|}{ Shift in one row } \\
\hline $0.00 a$ & -1.454 & -2.373 & 5.486 & -6.352 & $0.00 a$ & 0.1807 & -1.418 & 3.116 & -3.666 \\
\hline $0.04 a$ & -1.979 & -2.458 & 4.874 & -5.731 & $0.04 a$ & -0.0885 & -1.332 & 2.553 & -2.990 \\
\hline $0.08 a$ & -1.622 & -2.036 & 3.159 & -4.705 & $0.08 a$ & -0.0677 & -1.196 & 2.082 & -2.330 \\
\hline $0.12 a$ & -0.9657 & -1.519 & 2.177 & -4.273 & $0.12 a$ & -0.0132 & -0.9954 & 1.363 & -1.255 \\
\hline $0.16 a$ & -0.3879 & -1.259 & 2.159 & -2.871 & $0.16 a$ & 0.1263 & -1.050 & 1.641 & -1.547 \\
\hline $0.20 a$ & 0.0749 & -1.318 & 2.799 & -2.188 & $0.20 a$ & 0.3713 & -1.231 & 2.511 & -2.027 \\
\hline
\end{tabular}




\begin{tabular}{|l|l|l|l|l|l|l|l|l|l|}
\hline \multicolumn{9}{|c|}{ Shift in two rows } \\
\hline $0.00 a$ & -1.454 & -2.373 & 5.486 & -6.352 & $0.00 a$ & 0.1807 & -1.418 & 3.116 & -3.666 \\
\hline $0.04 a$ & -0.7649 & -1.190 & 1.282 & -5.444 & $0.04 a$ & -0.0338 & -0.8104 & 0.9339 & -1.0378 \\
\hline $0.08 a$ & -0.3413 & -0.5681 & 0.3025 & -0.3404 & $0.08 a$ & -0.0044 & -0.4778 & 0.3189 & -0.3273 \\
\hline $0.12 a$ & -0.1405 & -0.3322 & 0.1125 & -0.1196 & $0.12 a$ & -0.0096 & -0.3184 & 0.1580 & -0.1445 \\
\hline $0.16 a$ & -0.0037 & -0.2586 & 0.1123 & -0.0876 & $0.16 a$ & 0.0803 & -0.3010 & 0.1872 & -0.1410 \\
\hline $0.20 a$ & 0.1024 & -0.3007 & 0.1776 & -0.1436 & $0.20 a$ & 0.1766 & -0.3797 & 0.3506 & -0.2964 \\
\hline
\end{tabular}

In this study, the shift is introduced in the first and second rows, and the shift effect produced more impact when two rows were shifted. At lattice shifts $0.12 a$ and $0.16 a$, the impact is the highest in our observation. Even, the rows can be shifted further, but this shift causes the generation of original hexagonal lattice of the base structure.

\subsection{Effect of twist:}

Previously, dispersion tailoring was demonstrated by introducing twist effect in the lattice structure (Colman et al. 2012; Roy et al. 2012). In the twist induced PCW, the dispersion profile is controlled by achieving anti-crossing between even and odd modes. Similar to lattice shift, the twist introduced in the lattice directly impacts the dispersive curve and produces the flat band. Considering the previous results of different structures (Petrov and Eich 2004), the twist ( $\mathrm{t}$ ) in the lattice is introduced in the range of $0.00 a-0.20 a$ with $0.04 a$ step. From our preceding result, the radius of the holes and rings remains taken as $0.21 a$. Figure 9 shows the dispersion curves of the waveguide for both holes and rings structures. We introduced the twist in both first and second rows and studied the dispersion profile. The instituted twist has widened the group index-wavelength plot which is shown in the figure 10 . For instance, in case of ring like structure, almost a constant $n_{g}$ of 11.67 is observed over a bandwidth of $27.87 \mathrm{~nm}$ when the structure is affected by a twist parameter of $0.16 a$, compared to the base structure with $n_{g} 34.72$ over a bandwidth of $1.98 \mathrm{~nm}$. Table 3 gives the summary of applied twist, corresponding $n_{g}$ and the wavelength range for the PCWs with holes and rings.

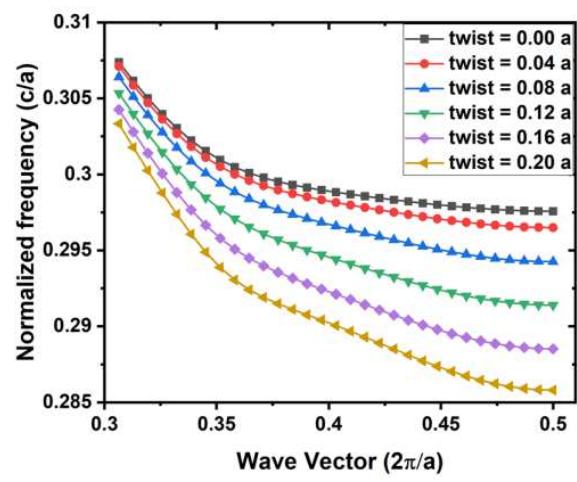

(a)

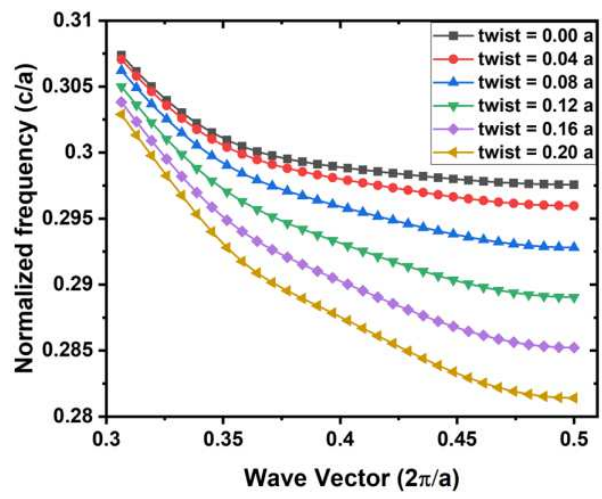

(b) 


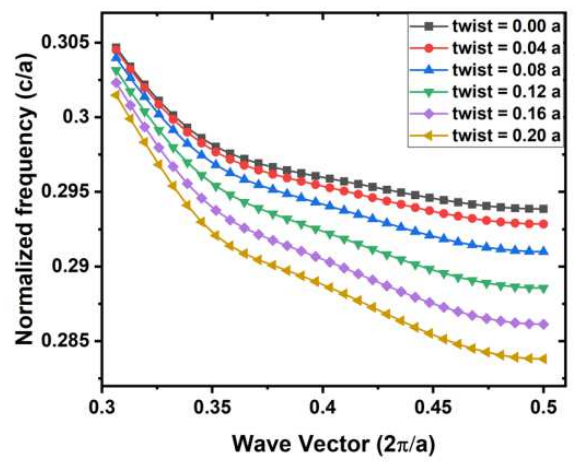

(c)

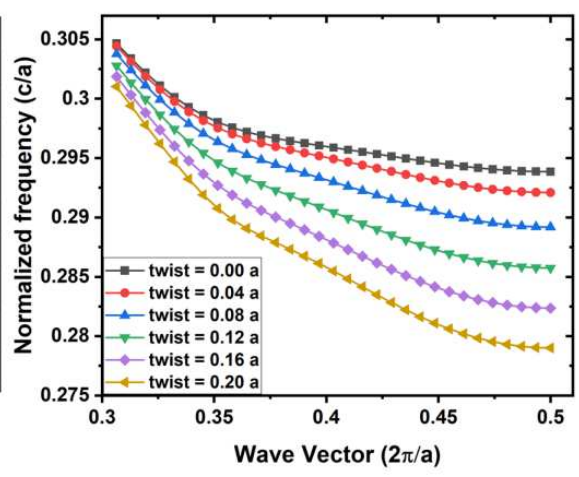

(d)

Fig. 9 Band 15 from the band diagram of PCW with holes and (a) twist in one row (b) twist in two rows. Band 15 from the band diagram of PCW with rings and (c) twist in one row (d) twist in two rows.

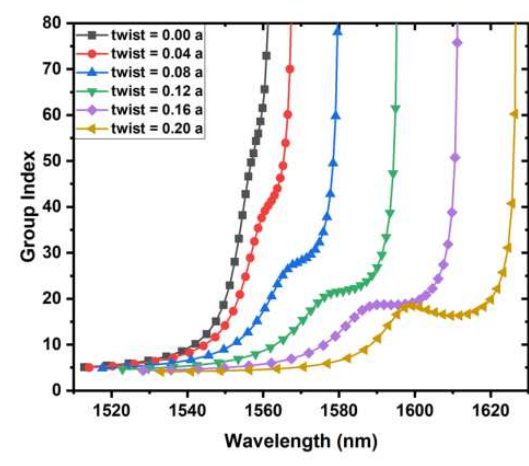

(a)

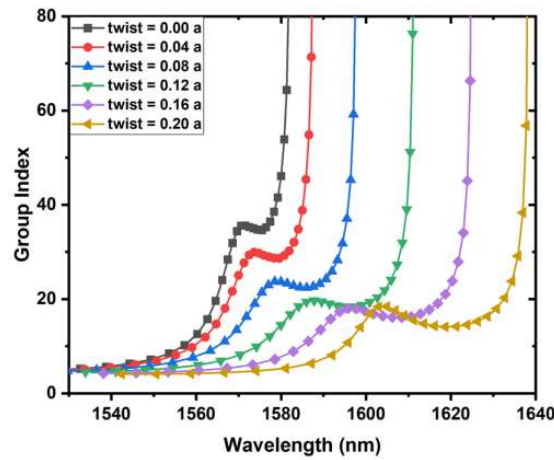

(c)

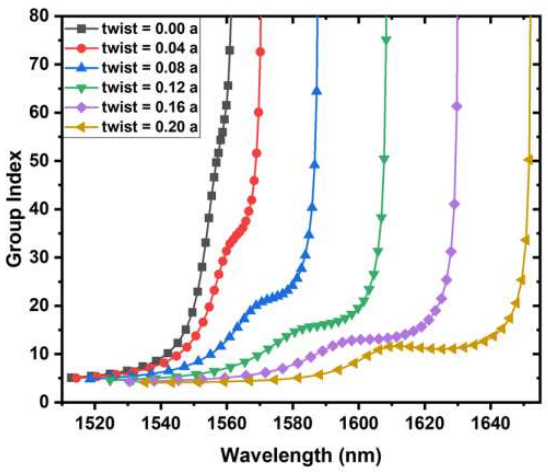

(b)

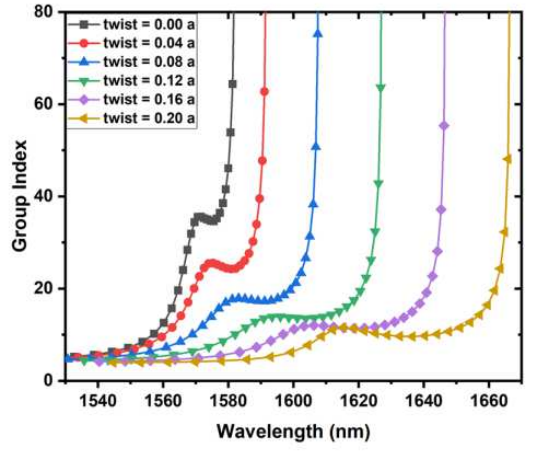

(d)

Fig. 10 Group index - wavelength plot of the PCW with holes and (a) twist in one row (b) twist in two rows. Group index - wavelength plot of the PCW with rings and (c) twist in one row (d) twist in two rows.

Table 3: Calculated group index and bandwidth of the waveguide at different twist values in first and second rows

\begin{tabular}{|l|l|l|l|l|l|}
\hline \multicolumn{3}{|c|}{ Holes } & \multicolumn{3}{c|}{ Rings } \\
\hline \multicolumn{1}{|c|}{ twist (t) } & \multicolumn{1}{|c|}{$\boldsymbol{n}_{\boldsymbol{g}}$} & $\begin{array}{c}\text { bandwidth } \\
(\mathbf{n m})\end{array}$ & twist (t) & $\boldsymbol{n}_{\boldsymbol{g}}$ & $\begin{array}{c}\text { bandwidth } \\
(\mathbf{n m})\end{array}$ \\
\hline \multicolumn{7}{|c|}{ Twist in one row } \\
\hline $0.00 a^{*}$ & - & - & $0.00 a$ & 34.72 & 1.98 \\
\hline $0.04 a^{*}$ & - & - & $0.04 a$ & 29.56 & 3.47 \\
\hline $0.08 a$ & 28.61 & 1.20 & $0.08 a$ & 22.60 & 4.64 \\
\hline $0.12 a$ & 22.40 & 3.11 & $0.12 a$ & 18.61 & 7.61 \\
\hline $\mathbf{0 . 1 6 a}$ & $\mathbf{1 8 . 7 2}$ & $\mathbf{1 1 . 2 9}$ & $\mathbf{0 . 1 6 a}$ & $\mathbf{1 6 . 4 3}$ & $\mathbf{1 0 . 9 7}$ \\
\hline $0.20 a$ & 16.59 & 8.7 & $0.20 a$ & 14.38 & 10.18 \\
\hline
\end{tabular}




\begin{tabular}{|l|l|l|l|l|l|}
\hline \multicolumn{7}{|c|}{ Twist in two rows } \\
\hline $0.00 a^{*}$ & - & - & $0.00 a$ & 34.72 & 1.98 \\
\hline $0.04 a^{*}$ & - & - & $0.04 a$ & 24.67 & 7.04 \\
\hline $0.08 a$ & 21.61 & 3.18 & $0.08 a$ & 17.51 & 15.96 \\
\hline $0.12 a$ & 15.58 & 4.46 & $0.12 a$ & 13.70 & 18.14 \\
\hline $0.16 a$ & 13.20 & 10.86 & $\mathbf{0 . 1 6 a}$ & $\mathbf{1 1 . 6 7}$ & $\mathbf{2 7 . 8 7}$ \\
\hline $\mathbf{0 . 2 0 a}$ & $\mathbf{1 1 . 3 4}$ & $\mathbf{2 8 . 9 1}$ & $0.20 a$ & 9.73 & 11.48 \\
\hline
\end{tabular}

*No flat band is observed with constant group index.

Like shift parameter, the twist in the lattice has directly controlled $\beta_{2}$ and $\beta_{4}$. Figure $11 \& 12$ shows the $\beta_{2}$ and $\beta_{4}$ variations with wavelength respectively. Here, one can have a choice to select the structure for different applications. One can select a high $n_{g}$ PCW with narrow bandwidth or a low $n_{g}$ PCW with a broad bandwidth. In these plots, the negative $\beta_{2} \&$ $\beta_{4}$ values and positive $\beta_{2} \& \beta_{4}$ values are observed for different wavelengths. Hence, the dispersion compensation can be achieved with in the waveguide. With the increase in twist from $0.00 a$ to $0.20 a$, the curve becomes more flat and the broad band is noticed with positive and negative peaks. Table 4 summarizes the applied twist, corresponding $\beta_{2}$ and $\beta_{4}$ values for holes and rings structures.

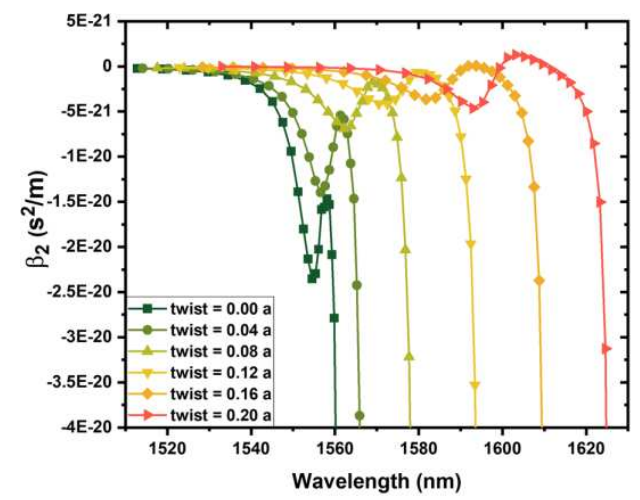

(a)

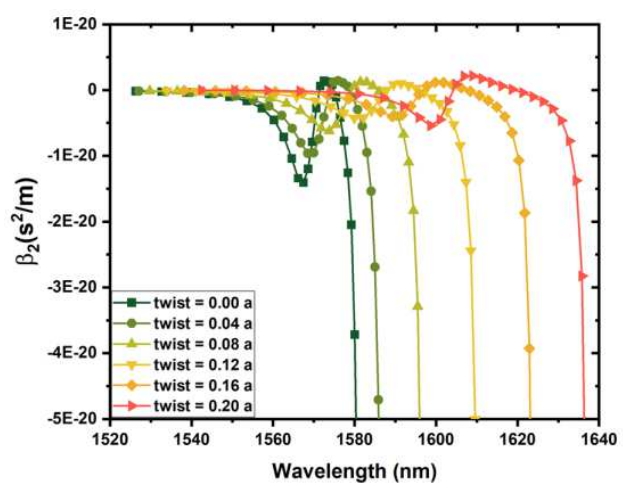

(c)

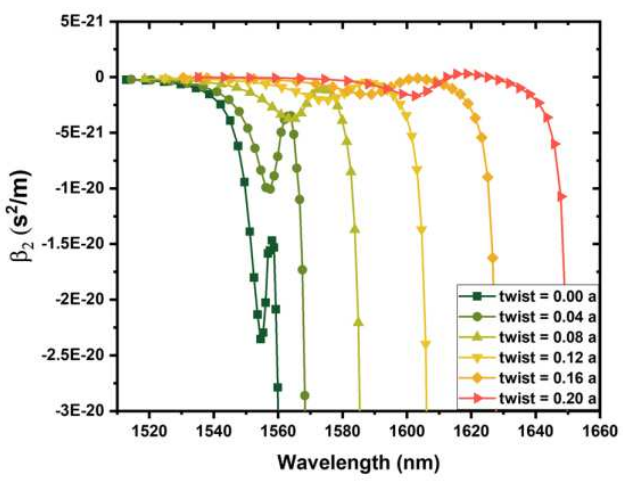

(b)

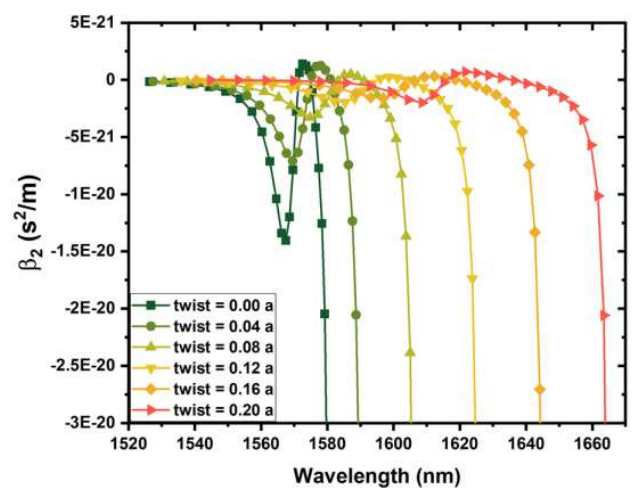

(d)

Fig. 11 Dispersion characteristics corresponding to figure 10 (a-d). (a) $\beta_{2}$ of PCW with holes and twist in one row (b) $\beta_{2}$ of PCW with holes and twist in two rows (c) $\beta_{2}$ of PCW with rings and twist in one row (d) $\beta_{2}$ of $\mathrm{PCW}$ with rings and twist in two rows. 


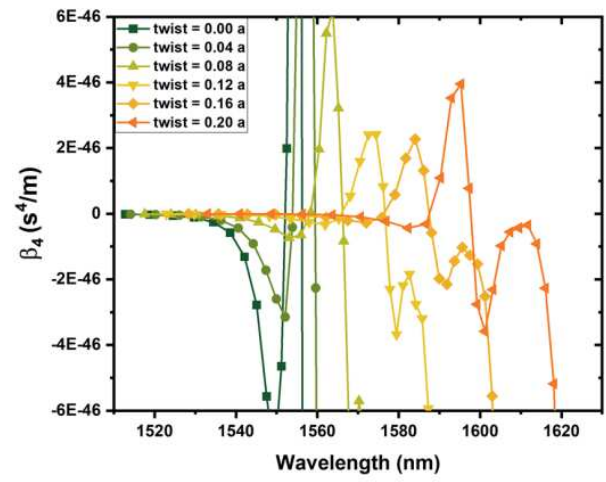

(a)

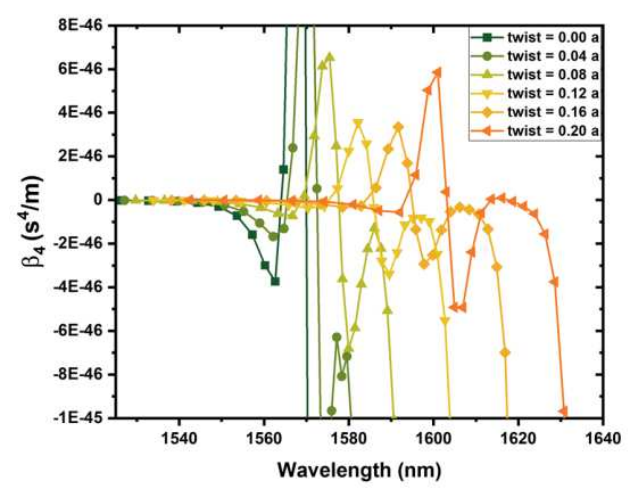

(c)

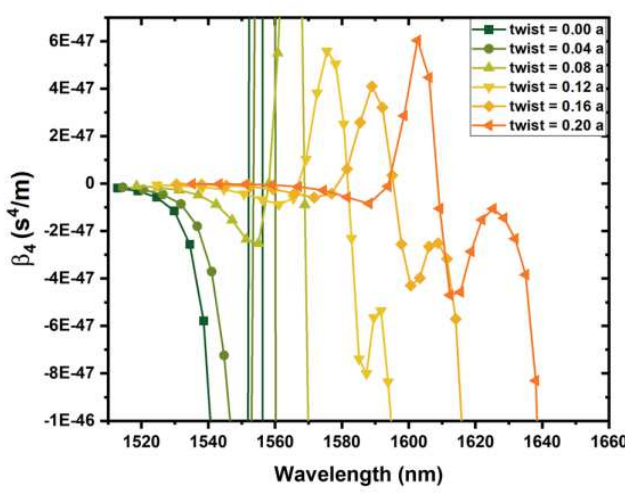

(b)

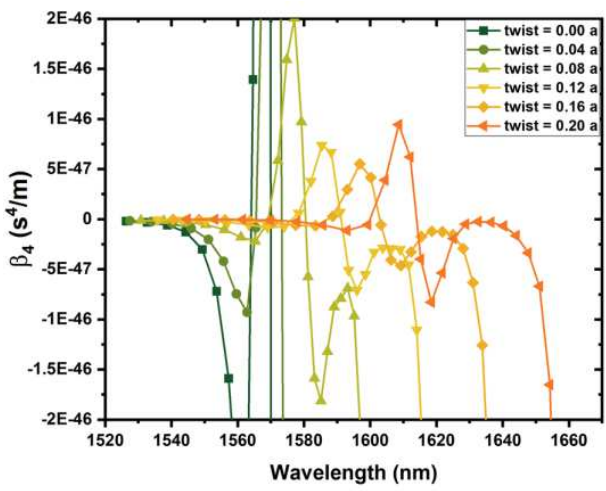

(d)

Fig. 12 Dispersion characteristics corresponding to figure 10 (a-d). (a) $\beta_{4}$ of PCW with holes and twist in one row (b) $\beta_{4}$ of PCW with holes and twist in two rows (c) $\beta_{4}$ of PCW with rings and twist in one row (d) $\beta_{4}$ of $\mathrm{PCW}$ with rings and twist in two rows.

Table 4: Calculated $\beta_{2}$ and $\beta_{4}$ values of the waveguide with applied twist

\begin{tabular}{|c|c|c|c|c|c|c|c|c|c|}
\hline \multicolumn{5}{|c|}{ Holes } & \multicolumn{5}{|c|}{ Rings } \\
\hline $\begin{array}{l}\text { twist } \\
\text { (t) }\end{array}$ & $\begin{array}{c}\beta_{2}(\max .) \\
\times 10^{-20} \\
\mathbf{s}^{2} / \mathbf{m} \\
\end{array}$ & $\begin{array}{c}\boldsymbol{\beta}_{2}(\min .) \\
\times 10^{-20} \\
\mathbf{s}^{2} / \mathbf{m}\end{array}$ & $\begin{array}{c}\beta_{4}(\max .) \\
\times 10^{-45} \\
\mathbf{s}^{4} / \mathbf{m}\end{array}$ & $\begin{array}{c}\beta_{4}(\min .) \\
\times 10^{-45} \\
\mathbf{s}^{4} / \mathbf{m} \\
\end{array}$ & $\begin{array}{l}\text { twist } \\
\text { (t) }\end{array}$ & $\begin{array}{c}\beta_{2}(\max .) \\
\times 10^{-20} \\
\mathbf{s}^{2} / \mathrm{m} \\
\end{array}$ & $\begin{array}{c}\beta_{2}(\min .) \\
\times 10^{-20} \\
\mathbf{s}^{2} / \mathbf{m}\end{array}$ & 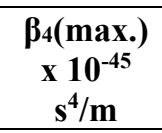 & $\begin{array}{c}\beta_{4}(\min .) \\
\times 10^{-45} \\
\mathbf{s}^{4} / \mathbf{m} \\
\end{array}$ \\
\hline \multicolumn{10}{|c|}{ Twist in one row } \\
\hline $0.00 a$ & -1.466 & -2.353 & 6.051 & -4.929 & $0.00 a$ & 0.1405 & -1.403 & 3.107 & -3.634 \\
\hline $0.04 a$ & -0.5429 & -1.396 & 1.910 & -3.594 & $0.04 a$ & 0.1432 & -0.9607 & 1.647 & -1.740 \\
\hline $0.08 a$ & -0.1703 & -0.6881 & 0.6186 & -0.7869 & $0.08 a$ & 0.1248 & -0.6192 & 0.6526 & -0.6805 \\
\hline $0.12 a$ & -0.0649 & -0.4145 & 0.2414 & -0.3680 & $0.12 a$ & 0.1025 & -0.4269 & 0.3555 & -0.3395 \\
\hline $0.16 a$ & 0.0056 & -0.3641 & 0.2270 & -0.2148 & $0.16 a$ & 0.1172 & -0.4029 & 0.3347 & -0.2935 \\
\hline $0.20 a$ & 0.1284 & -0.4644 & 0.3953 & -0.3582 & $0.20 a$ & 0.2176 & -0.5393 & 0.5856 & -0.4924 \\
\hline \multicolumn{10}{|c|}{ Twist in two rows } \\
\hline $0.00 a$ & -1.466 & -2.353 & 6.051 & -4.929 & $0.00 a$ & 0.1405 & -1.403 & 3.10759 & -3.634 \\
\hline $0.04 a$ & -0.3467 & -1.005 & 1.172 & -2.364 & $0.04 a$ & 0.1249 & -0.7096 & 0.904635 & -0.8689 \\
\hline $0.08 a$ & -0.1038 & -0.3732 & 0.1993 & -0.2038 & $0.08 a$ & 0.0488 & -0.3278 & 0.200412 & -0.1813 \\
\hline $0.12 a$ & -0.0443 & -0.2053 & 0.0557 & -0.0801 & $0.12 a$ & 0.0249 & -0.1959 & 0.0737149 & -0.0707 \\
\hline $0.16 a$ & -0.0113 & -0.1543 & 0.0410 & -0.0429 & $0.16 a$ & 0.0312 & -0.1604 & 0.0551592 & -0.0462 \\
\hline $0.20 a$ & 0.0298 & -0.1683 & 0.0603 & -0.0469 & $0.20 a$ & 0.0708 & -0.1995 & 0.0944128 & -0.0828 \\
\hline
\end{tabular}

\subsection{Combined effect of change in radius, lattice shift and twist:}

To combine the impact of all the geometrical parameters on the features of PCW, the change in radius, lattice shift and twist were introduced in the structure. For this purpose, in the input 
program, the shift ranging from $0.00 a$ to $0.20 a$, twist ranging from $0.00 a$ to $0.20 a$ and radius of $0.21 a$ were introduced. The dispersion curves were modified with these geometrical changes, and at $\mathrm{s}=0.16 a, \mathrm{t}=0.16 a$ and $\mathrm{r}=0.21 a$, the structure has resulted with dispersive curves of highest slope. Figure 13 shows the band 15 from the photonic band diagram of the PCW with holes and rings structures.

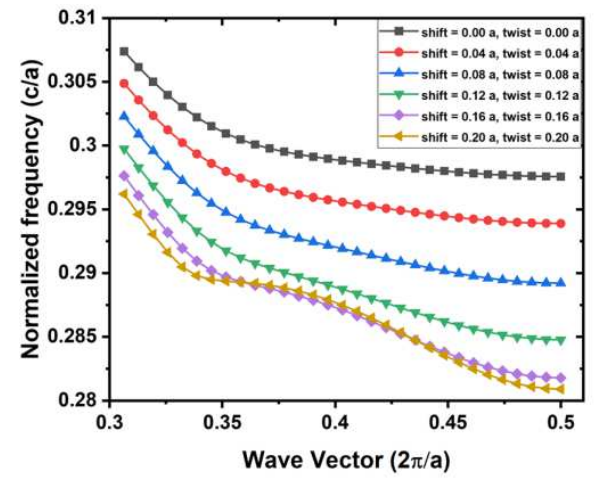

(a)

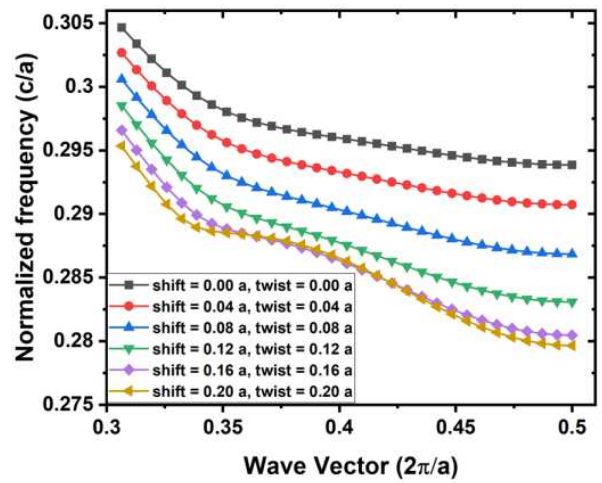

(c)

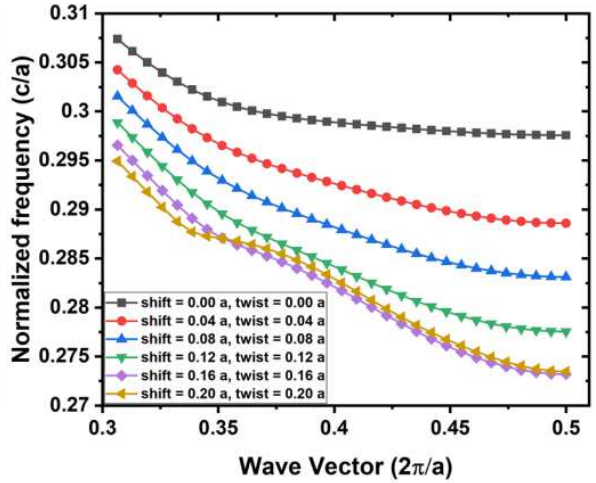

(b)

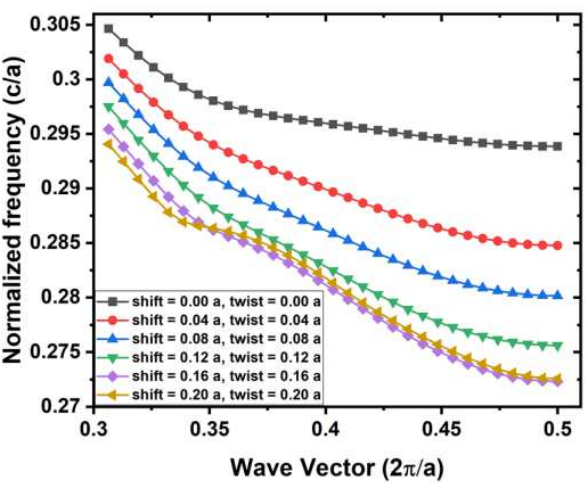

(d)

Fig. 13 Band 15 from the band diagram of PCW with holes and applied (a) shift and twist in one row (b) shift and twist in two rows. Band 15 from the band diagram of PCW with rings and applied (c) shift and twist in one row (d) shift and twist in two rows.

Figure 14 shows the change in group index with wavelength for all the structures. A nearly constant group index is observed over a wide range with $\mathrm{s}=\mathrm{t}=0.16 a$ and $\mathrm{r}=0.21 a$ when only first row is spatially changed. This flatness is further increased when the shift and twist were introduced in the second row of holes/rings. However, the group index dropped to a lower value. Here, the sudden kink in the group index plots is due to the closeness of the bands in the band structure. Table 5 gives the summary of $n_{g}$ values and the bandwidth that the $n_{g}$ is maintained almost constant due to the lattice shift and twist. 


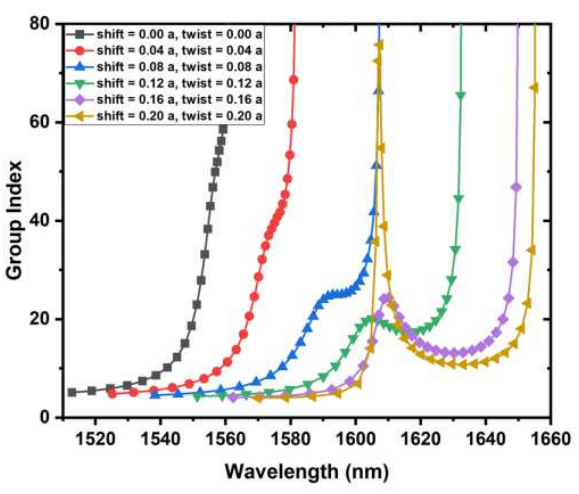

(a)

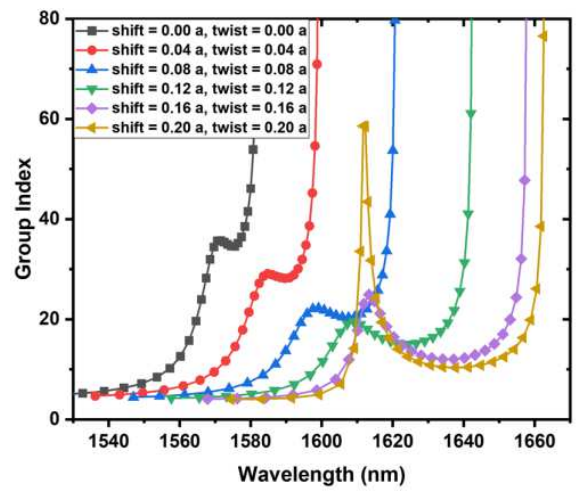

(c)

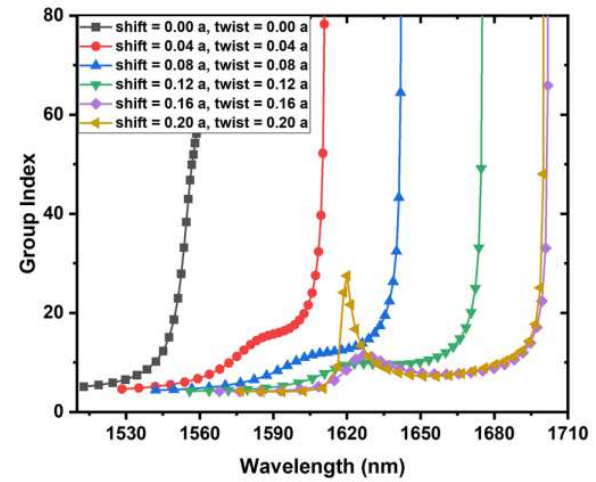

(b)

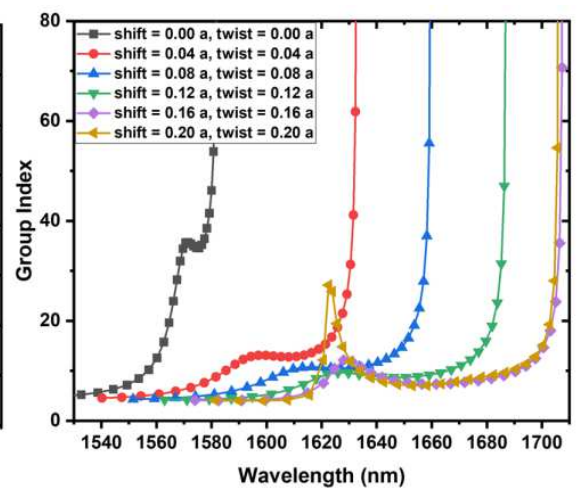

(d)

Fig. 14 Group index - wavelength plot of the PCW with holes and applied (a) shift and twist in one row (b) shift and twist in two rows. Group index - wavelength plot of the PCW with rings and applied (c) shift and twist in one row (d) shift and twist in two rows.

Table 5: Calculated group index and bandwidth of the waveguide at different shift and twist values (combined) in first and second rows

\begin{tabular}{|l|l|l|l|l|l|}
\hline \multicolumn{3}{|c|}{ Holes } & \multicolumn{3}{c|}{ Rings } \\
\hline $\begin{array}{c}\text { shift (s) } \\
\text { and } \\
\text { twist (t) }\end{array}$ & $\boldsymbol{n}_{\boldsymbol{g}}$ & $\begin{array}{c}\text { bandwidth } \\
\text { (nm) }\end{array}$ & $\begin{array}{c}\text { shift (s) } \\
\text { and } \\
\text { twist (t) }\end{array}$ & $\boldsymbol{n}_{\boldsymbol{g}}$ & $\begin{array}{c}\text { bandwidth } \\
\text { (nm) }\end{array}$ \\
\hline \multicolumn{5}{|c|}{ Shift and twist in one row } \\
\hline $0.00 a^{*}$ & - & - & $0.00 a$ & 34.71 & 1.98 \\
\hline $0.04 a^{*}$ & - & - & $0.04 a$ & 28.51 & 6.16 \\
\hline $0.08 a$ & 24.61 & 4.27 & $0.08 a$ & 20.61 & 6.98 \\
\hline $0.12 a$ & 17.61 & 8.26 & $0.12 a$ & 15.33 & 9.61 \\
\hline $\mathbf{0 . 1 6} \boldsymbol{a}$ & $\mathbf{1 3 . 3 7}$ & $\mathbf{1 1 . 1 1}$ & $\mathbf{0 . 1 6} a$ & $\mathbf{1 2 . 3 7}$ & $\mathbf{1 5 . 1 4}$ \\
\hline $0.20 a$ & 10.86 & 6.84 & $0.20 a$ & 10.57 & 14.18 \\
\hline \multicolumn{7}{|c|}{ Shift and twist in two rows } \\
\hline $0.00 a^{*}$ & - & - & $0.00 a$ & 34.72 & 1.98 \\
\hline $0.04 a$ & 15.37 & 4.55 & $0.04 a$ & 12.89 & 11.11 \\
\hline $0.08 a$ & 12.25 & 11.81 & $0.08 a$ & 10.61 & 24.04 \\
\hline $0.12 a$ & 9.68 & 26.70 & $0.12 a$ & 8.86 & 21.39 \\
\hline $\mathbf{0 . 1 6} a$ & $\mathbf{7 . 8 8}$ & $\mathbf{2 9 . 4 2}$ & $\mathbf{0 . 1 6 a}$ & $\mathbf{7 . 6 4}$ & $\mathbf{3 0 . 4 1}$ \\
\hline $0.20 a$ & 7.49 & 25.64 & $0.20 a$ & 7.39 & 26.08 \\
\hline
\end{tabular}

*No flat band is observed with constant group index.

The figure $15 \& 16$ gives the $\beta_{2}$ and $\beta_{4}$ profile of holes and rings structures with the induced shift and twist. In these plots, the $\beta_{2}$ and $\beta_{4}$ were changing sharply between positive 
and negative values. This change in $\beta_{2}$ and $\beta_{4}$ between positive and negative values confirms the dispersion compensation in PCW.

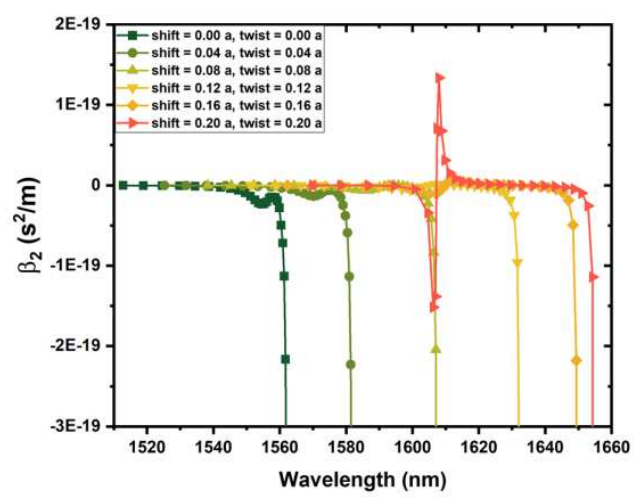

(a)

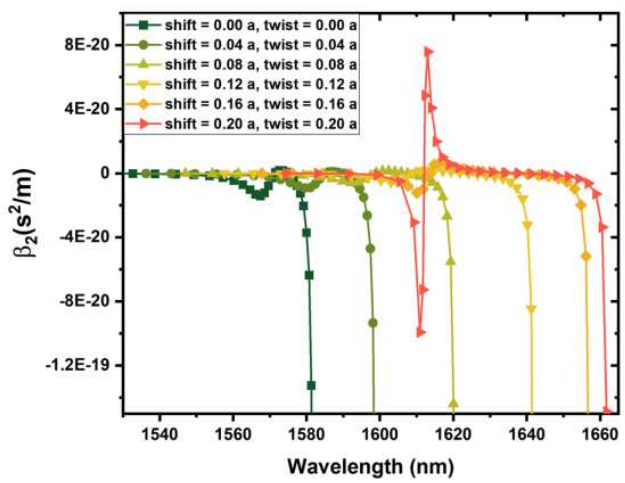

(c)

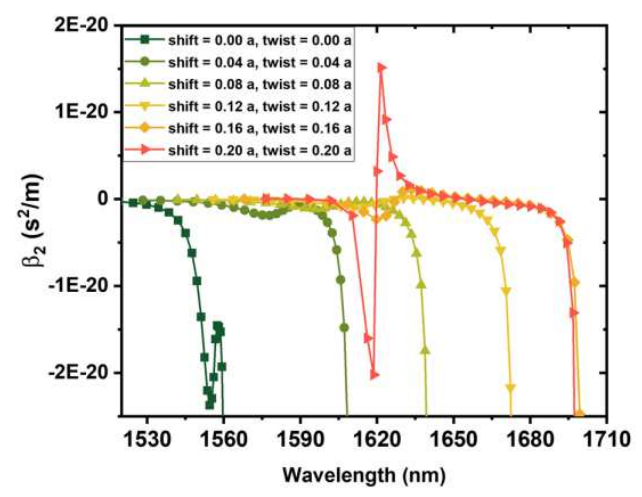

(b)

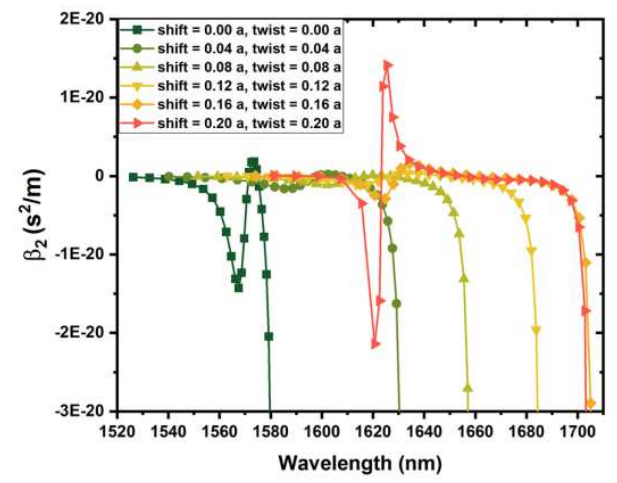

(d)

Fig.15 Dispersion characteristics corresponding to figure 14 (a-d). $\beta_{2}$ of PCW with holes and applied (a) shift and twist in one row (b) shift and twist in two rows. $\beta_{2}$ of PCW with rings and applied (c) shift and twist in one row (d) shift and twist in two rows.

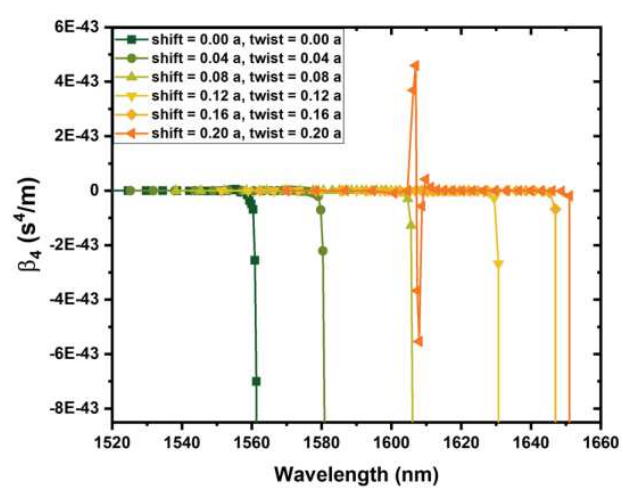

(a)

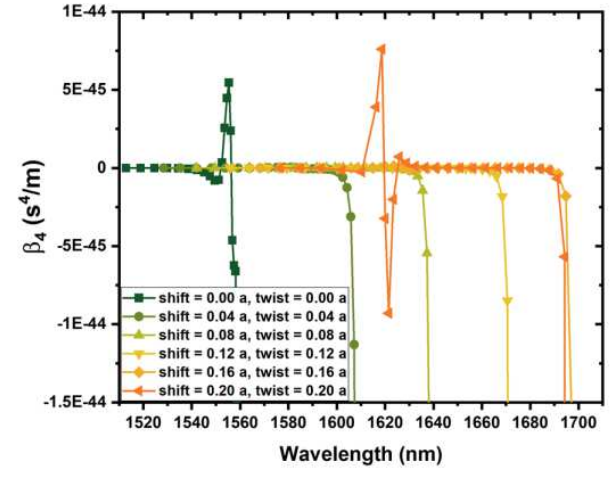

(b) 


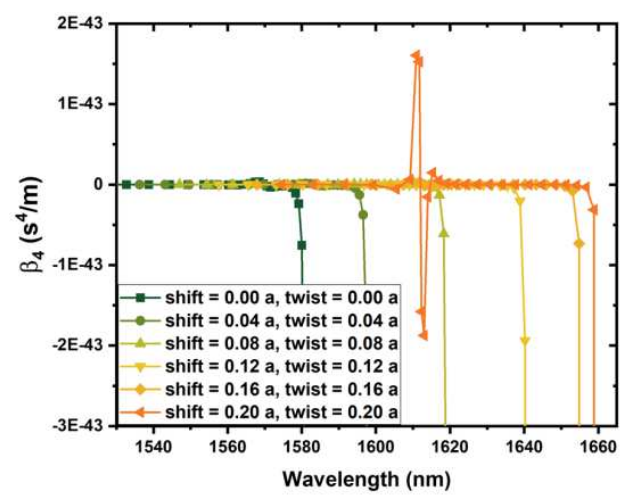

(c)

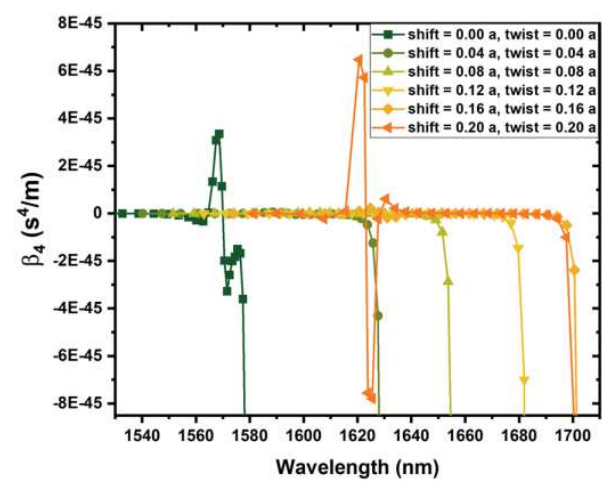

(d)

Fig. 16 Dispersion characteristics corresponding to figure 14 (a-d). $\beta_{4}$ of PCW with holes and applied (a) shift and twist in one row (b) shift and twist in two rows. $\beta_{4}$ of PCW with rings and applied (c) shift and twist in one row (d) shift and twist in two rows.

In these figures the negative and positive $\beta_{2} \& \beta_{4}$ values are observed for different wavelengths. Therefore, the dispersion compensation can be achieved by the serial cascading of the two sections of such waveguide with opposite $\beta_{2} \& \beta_{4}$ values as discussed by Hou et al. (2009). Table 6 gives the complete summary of applied shift, twist and corresponding $\beta_{2}$ and $\beta_{4}$ values for holes and rings structures.

Table 6: Calculated $\beta_{2}$ and $\beta_{4}$ values of the waveguide with combined shift and twist

\begin{tabular}{|c|c|c|c|c|c|c|c|c|c|}
\hline \multicolumn{5}{|c|}{ Holes } & \multicolumn{5}{|c|}{ Rings } \\
\hline $\begin{array}{c}\text { shift (s) } \\
\text { and } \\
\text { twist (t) }\end{array}$ & $\begin{array}{c}\boldsymbol{\beta}_{2}(\max .) \\
\times 10^{-20} \\
\mathbf{s}^{2} / \mathbf{m}\end{array}$ & $\begin{array}{c}\boldsymbol{\beta}_{2}(\min .) \\
\times 10^{-20} \\
s^{2} / m\end{array}$ & $\begin{array}{c}\beta_{4}(\max .) \\
\times 10^{-45} \\
\mathbf{s}^{4} / \mathbf{m}\end{array}$ & $\begin{array}{c}\beta_{4}(\min .) \\
\times 10^{-45} \\
\mathbf{s}^{4} / \mathbf{m}\end{array}$ & $\begin{array}{l}\text { shift (s) } \\
\text { and } \\
\text { twist (t) }\end{array}$ & $\begin{array}{c}\boldsymbol{\beta}_{2}(\max .) \\
\times 10^{-20} \\
\mathbf{s}^{2} / \mathbf{m}\end{array}$ & $\begin{array}{c}\beta_{2}(\min .) \\
\times 10^{-20} \\
s^{2} / m\end{array}$ & $\begin{array}{c}\beta_{4}(\max .) \\
\times 10^{-45} \\
\mathbf{s}^{4} / \mathbf{m}\end{array}$ & $\begin{array}{c}\beta_{4}(\min .) \\
\times 10^{-45} \\
\mathbf{s}^{4} / \mathbf{m}\end{array}$ \\
\hline \multicolumn{10}{|c|}{ Shift and twist in one row } \\
\hline $0.00 a$ & -1.455 & -2.374 & 5.463 & -6.242 & $0.00 a$ & 0.1817 & -1.427 & 3.350 & -3.278 \\
\hline $0.04 a$ & -0.5733 & -1.362 & 1.904 & -2.051 & $0.04 a$ & 0.1042 & -0.9210 & 1.493 & -1.434 \\
\hline $0.08 a$ & -0.0124 & -0.6058 & 0.6210 & -0.5677 & $0.08 a$ & 0.1438 & -0.5893 & 0.5994 & -0.5480 \\
\hline $0.12 a$ & 0.1568 & -0.5144 & 0.4670 & -0.4057 & $0.12 a$ & 0.2153 & -0.5400 & 0.5973 & -0.5207 \\
\hline $0.16 a$ & 0.5627 & -1.091 & 2.818 & -2.323 & $0.16 a$ & 0.6742 & -1.233 & 3.026 & -3.156 \\
\hline $0.20 a$ & 13.36 & -15.51 & 459.5 & -553.3 & $0.20 a$ & 7.584 & -9.919 & 160.4 & -187.5 \\
\hline \multicolumn{10}{|c|}{ Shift and twist in two rows } \\
\hline $0.00 a$ & -1.455 & -2.374 & 5.463 & -6.242 & $0.00 a$ & 0.1817 & -1.427 & 3.350 & -3.278 \\
\hline $0.04 a$ & -0.0608 & -0.1876 & 0.0480 & -0.0556 & $0.04 a$ & 0.0222 & -0.1616 & 0.0527 & -0.0487 \\
\hline $0.08 a$ & -0.0312 & -0.1136 & 0.0176 & -0.0222 & $0.08 a$ & 0.0117 & -0.1118 & 0.0249 & -0.0222 \\
\hline $0.12 a$ & 0.0118 & -0.0970 & 0.0217 & -0.0191 & $0.12 a$ & 0.0332 & -0.1068 & 0.0317 & -0.0270 \\
\hline $0.16 a$ & 0.1114 & -0.2317 & 0.1245 & -0.1206 & $0.16 a$ & 0.1557 & -0.2888 & 0.2158 & -0.1781 \\
\hline $0.20 a$ & 1.513 & -2.021 & 7.605 & -9.298 & $0.20 a$ & 1.414 & -2.139 & 6.472 & -7.795 \\
\hline
\end{tabular}

\section{Conclusions:}

In conclusion, we have revisited the effect of geometrical parameters on the properties of a line-defect PCW in a systematic way. The effect of change in radius of holes and rings, effect of lattice shift and twist, and the combined effect of all three were studied. The perturbation is introduced in the structure of PCW in such a way that the dispersion curve can 
be controlled over a desired bandwidth with desired group index. This has increased the degrees of freedom to control the dispersive curve. This dispersion engineering has also enhanced the group index - bandwidth product. Based on this study, one can design a waveguide with nearly constant and low $n_{g}$ over a broad wavelength range or a waveguide with nearly constant and high $n_{g}$ with narrow band. The second and fourth order dispersion curves were also under control with the introduced perturbation. Introducing the twist and shift in the second row of the PCW further elevated the studying parameters. This study can help in designing application specific devices like optical buffer memories, optical switches, to generate efficient nonlinear effects such as super continuum, and devices with desired slow light features.

\section{Acknowledgements:}

Authors sincerely thank Department of Science and Technology, Government of India for funding this work through INSPIRE fellowship program (IF160435).

\section{Declarations:}

Funding:

This work is funded by Department of Science and Technology, Government of India (DST-GoI) under the INSPIRE fellowship with fellowship number IF160435. Authors sincerely thank DST-GoI for the same.

\section{Conflicts of interest:}

The authors declare that they have no known competing financial interests or personal relationships that could have appeared to influence the work reported in this paper.

\section{Availability of data and material:}

Not applicable

\section{Code availability:}

Not applicable

\section{Authors' contributions:}

V D R Pavan has performed the simulations, collected the raw data, did the primary analysis and prepared the manuscript. S. Roy has analyzed the results, drawn the conclusions and finalized the contents of the manuscript.

\section{References:}

Schulz, S.A., O'Faolain, L., Beggs, D.M., White, T.P., Melloni, A., Krauss, T.F.: Dispersion engineered slow light in photonic crystals: a comparison. J. Opt. 12(2010), 104004 (2010) 
Baba, T.: Slow light in photonic crystals. Nat. Photonics (2008).

https://doi.org/10.1038/nphoton.2008.146

Krauss, T.F.: Slow light in photonic crystal waveguides. J. Phys. D. Appl. Phys. (2007).

https://doi.org/10.1088/0022-3727/40/9/S07

Letartre, X., Seassal, C., Grillet, C., Rojo-Romeo, P., Viktorovitch, P., Le Vassor D’Yerville, M., Cassagne, D., Jouanin, C.: Group velocity and propagation losses measurement in a single-line photonic-crystal waveguide on InP membranes. Appl. Phys. Lett. (2001).

https://doi.org/10.1063/1.1405146

Vlasov, Y.A., O’Boyle, M., Hamann, H.F., McNab, S.J.: Active control of slow light on a chip with photonic crystal waveguides. Nature (2005). https://doi.org/10.1038/nature04210

Soljačić, M., Joannopoulos, J.D.: Enhancement of nonlinear effects using photonic crystals. Nat. Mater. (2004). https://doi.org/10.1038/nmat1097

Li, J.T., Zhou, J.Y.: Nonlinear optical frequency conversion with stopped short light pulses. Opt. Express (2006). https://doi.org/10.1364/OE.14.002811

Colman, P., Combrié, S., Lehoucq, G., De Rossi, A.: Control of dispersion in photonic crystal waveguides using group symmetry theory. Opt. Express (2012). https://doi.org/10.1364/oe.20.013108

Ebnali-Heidari, M., Grillet, C., Monat, C., Eggleton, B.J.: Dispersion engineering of slow light photonic crystal waveguides using microfluidic infiltration. Opt. Express (2009).

https://doi.org/10.1364/OE.17.001628

Hou, J., Gao, D., Wu, H., Hao, R., Zhou, Z.: Flat band slow light in symmetric line defect photonic crystal waveguides. IEEE Photon. Technol. Lett. (2009). https://doi.org/10.1109/LPT.2009.2030160

Roy, S., Willinger, A., Combrié, S., De Rossi, A., Eisenstein, G., Santagiustina, M.: Narrowband optical parametric gain in slow mode engineered GaInP photonic crystal waveguides. Opt. Lett. (2012). https://doi.org/10.1364/OL.37.002919

Roy, S., Santagiustina, M., Colman, P., Combrié, S., De Rossi, A.: Modeling the dispersion of the nonlinearity in slow mode photonic crystal waveguides. IEEE Photonics J. (2012).

https://doi.org/10.1109/JPHOT.2011.2181942

Frandsen, L.H., Lavrinenko, A. V, Fage-Pedersen, J., Borel, P.I.: Photonic crystal waveguides with semi-slow light and tailored dispersion properties. Opt. Express (2006).

https://doi.org/10.1364/OE.14.009444

Petrov, A.Y., Eich, M.: Zero dispersion at small group velocities in photonic crystal waveguides. Appl. Phys. Lett. (2004). https://doi.org/10.1063/1.1815066

Li, J., White, T.P., O'Faolain, L., Gomez-Iglesias, A., Krauss, T.F.: Systematic design of flat band slow light in photonic crystal waveguides. Opt. Express (2008). https://doi.org/10.1364/OE.16.006227

Hamachi, Y., Kubo, S., Baba, T.: Slow light with low dispersion and nonlinear enhancement in a lattice-shifted photonic crystal waveguide. Opt. Lett. (2009). https://doi.org/10.1364/OL.34.001072 
Säynätjoki, A., Mulot, M., Ahopelto, J., Lipsanen, H.: Dispersion engineering of photonic crystal waveguides with ring-shaped holes. Opt. Express (2007). https://doi.org/10.1364/OE.15.008323

Casas-Bedoya, A., Husko, C., Monat, C., Grillet, C., Gutman, N., Domachuk, P., Eggleton, B.J.: Slow-light dispersion engineering of photonic crystal waveguides using selective microfluidic infiltration. Opt. Lett. (2012). https://doi.org/10.1364/OL.37.004215

Johnson, S. G., Joannopoulos, J. D.: Block-iterative frequency-domain methods for Maxwell's equations in a planewave basis. Opt. Express (2001). https://doi.org/10.1364/OE.8.000173 and http://ab-initio.mit.edu/mpb/doc/mpb.pdf

Notomi, M., Yamada, K., Shinya, A., Takahashi, J., Takahashi, C., Yokohama, I.: Extremely large group-velocity dispersion of line-defect waveguides in photonic crystal slabs. Phys. Rev. Lett. (2001). https://doi.org/10.1103/PhysRevLett.87.253902

Bagci, F., Akaoglu, B.: Effects of innermost rows on slow light properties of photonic crystal waveguides. IEEE Xplore (2013). https://ieeexplore.ieee.org/document/6608281

Tang, J., Wang, T., Li, X., Wang, B., Dong, C., Gao, L., Liu, B., He, Y., Yan, W.: Wideband and low dispersion slow light in lattice-shifted photonic crystal waveguides. J. Light. Technol. (2013). https://doi.org/10.1109/JLT.2013.2280716

Yang, D., Tian, H., Ji, Y.: The properties of lattice-shifted microcavity in photonic crystal slab and its applications for electro-optical sensor. Sens. Actuator A Phys. (2011).

https://doi.org/10.1016/j.sna.2011.08.001 


\section{Figures}

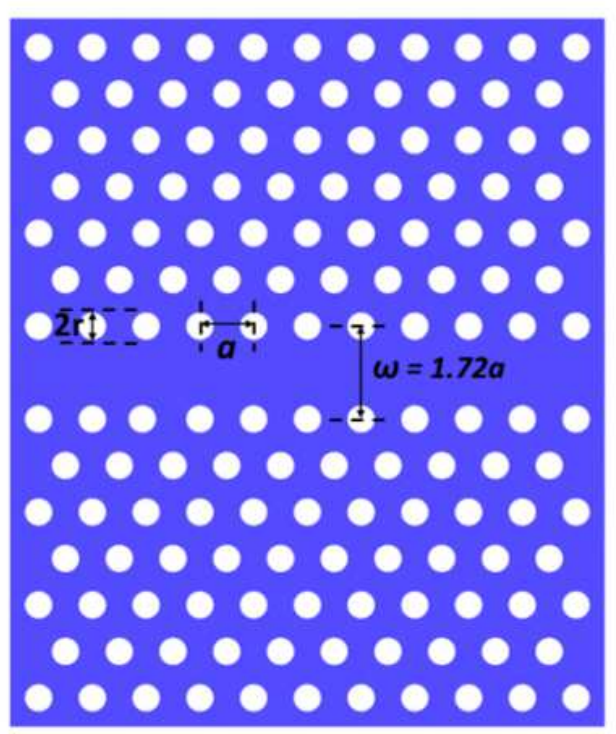

(a)
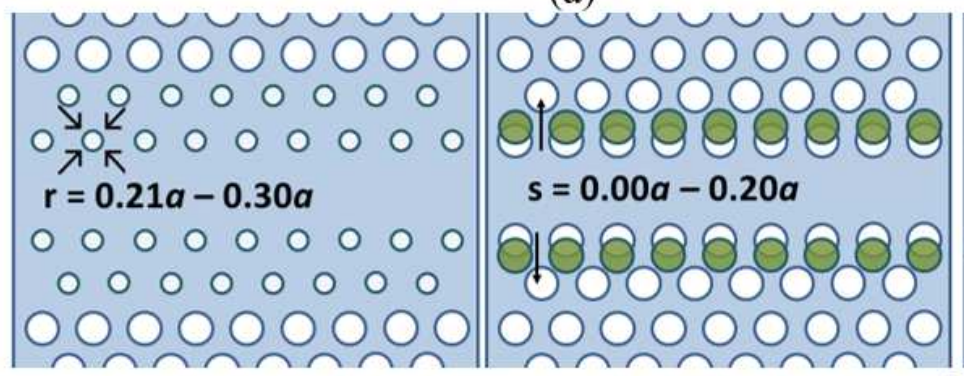

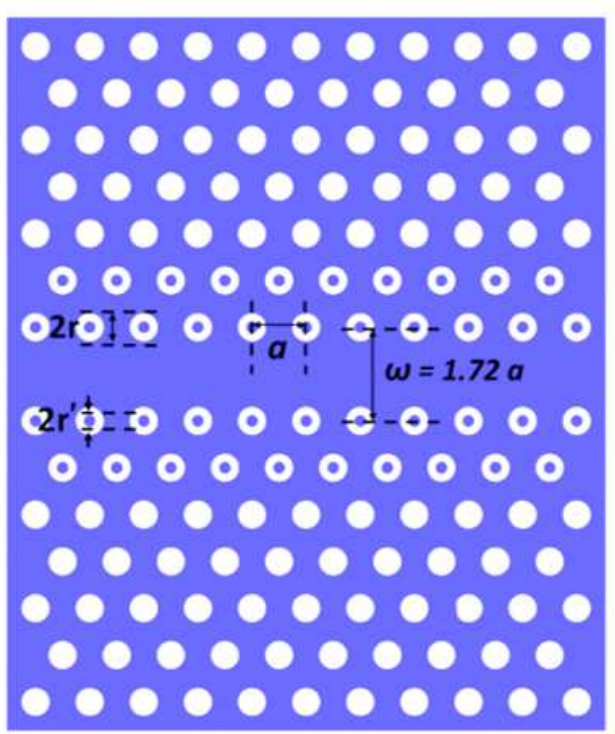

(b)

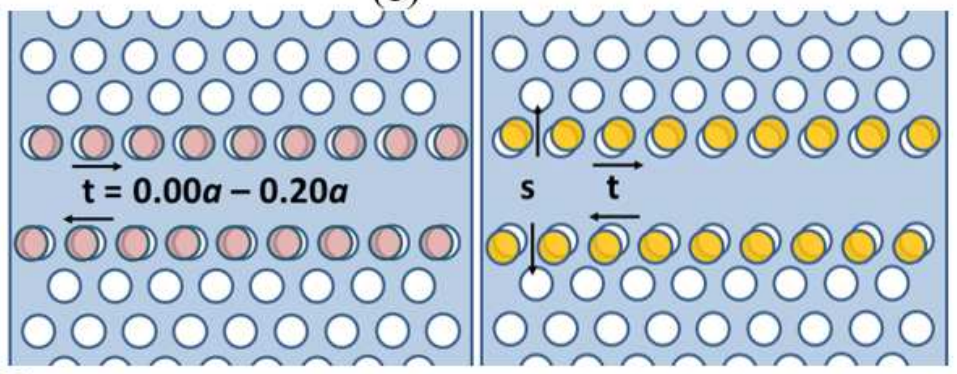

(c)

\section{Figure 1}

Schematic of the structure. (a) 2-D hexagonal lattice with air cylinders to form the waveguide. (b) waveguide with rings (c) illustration of change in radius, introducing shift and twist effects. 


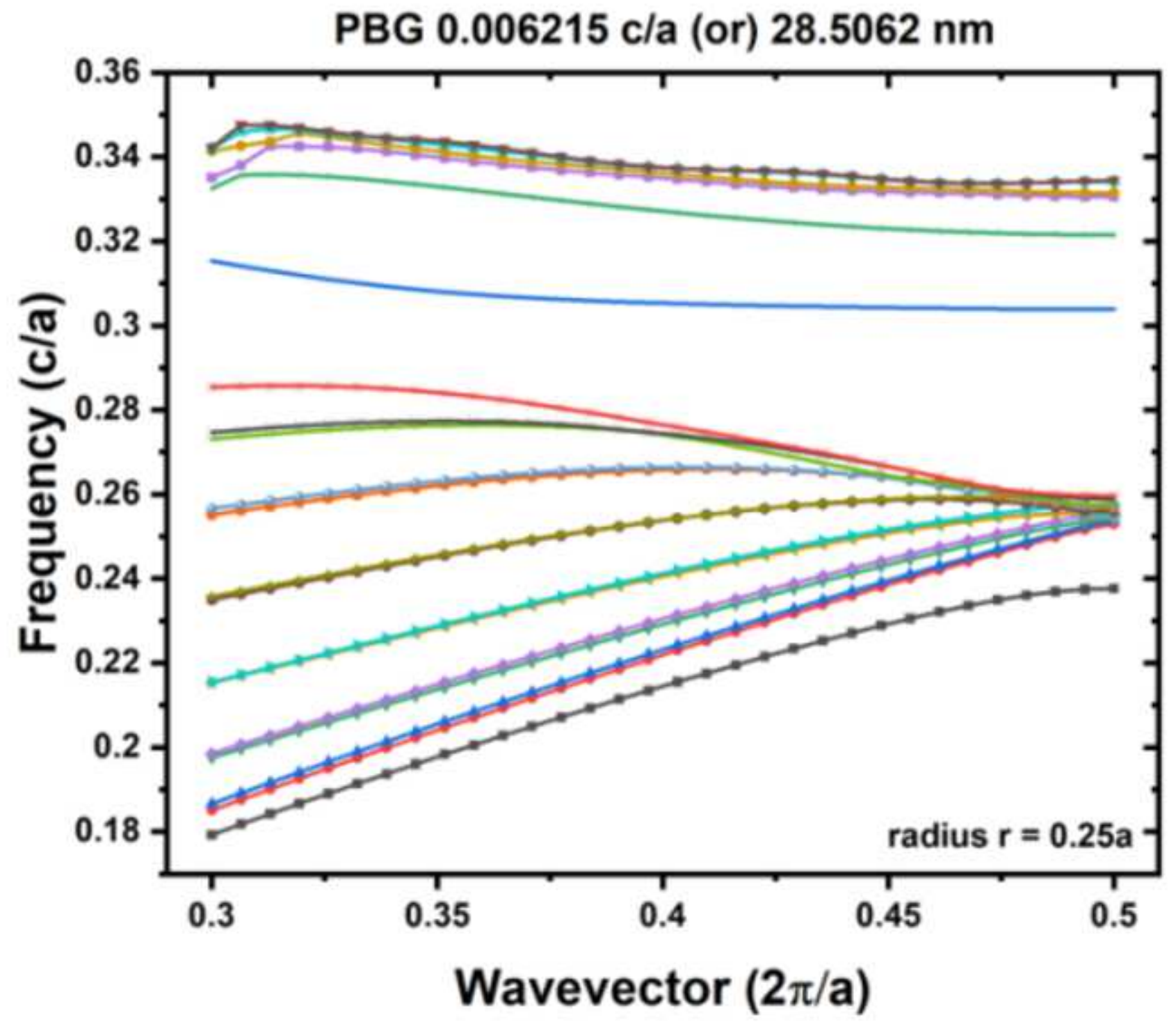

Figure 2

Photonic band diagram of the structure. The PCW structure with hole radius $r=0.25 a$ is taken as a reference in the study. 


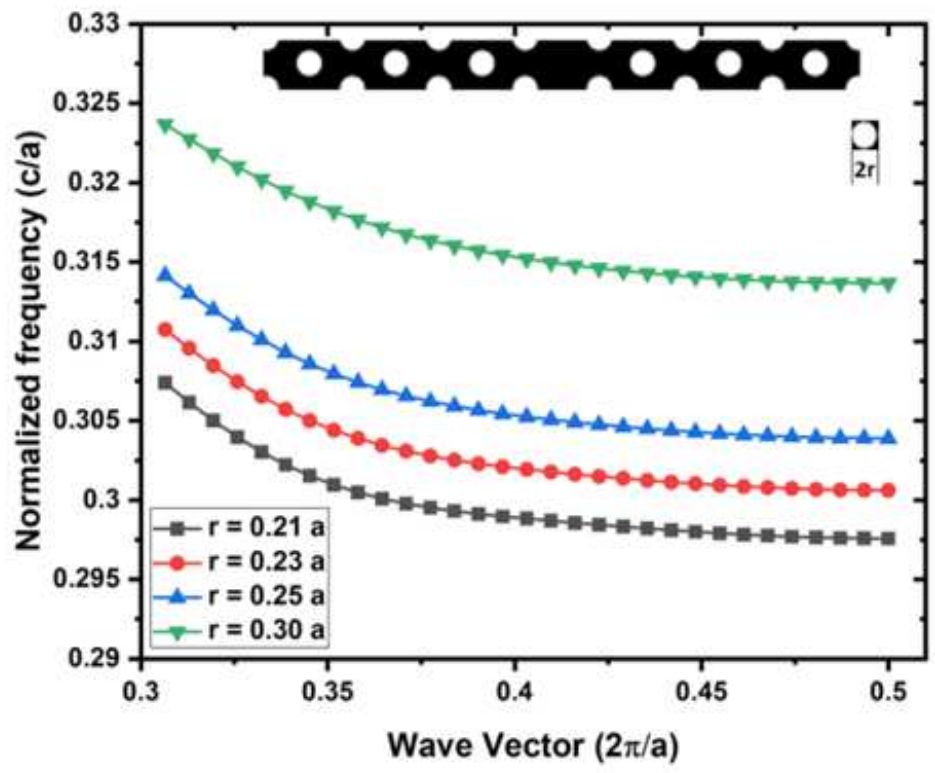

(a)

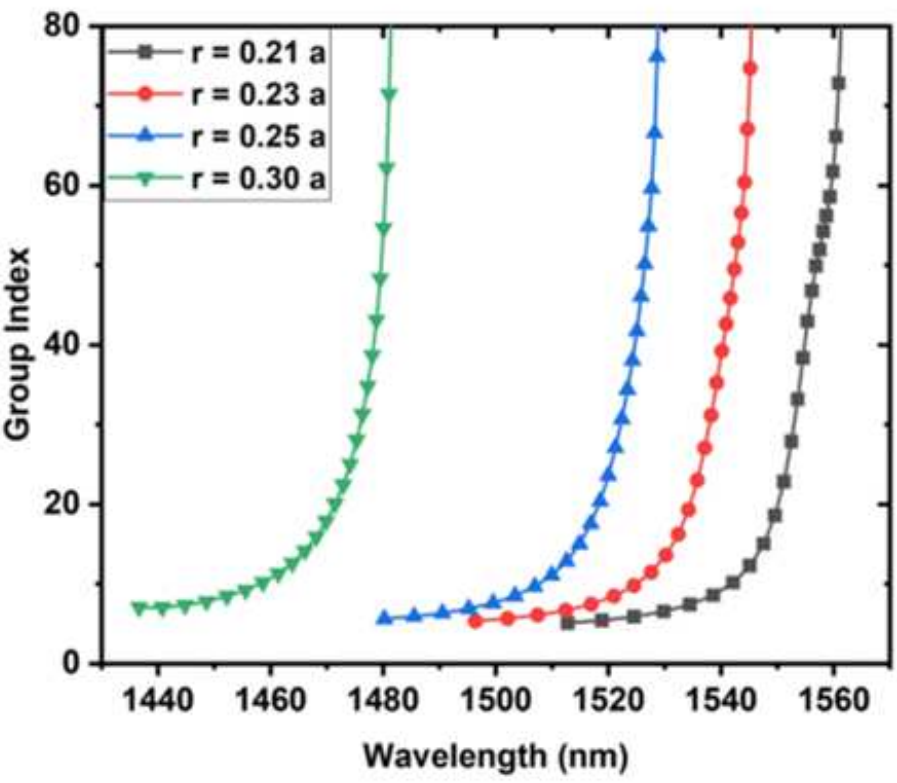

(c)

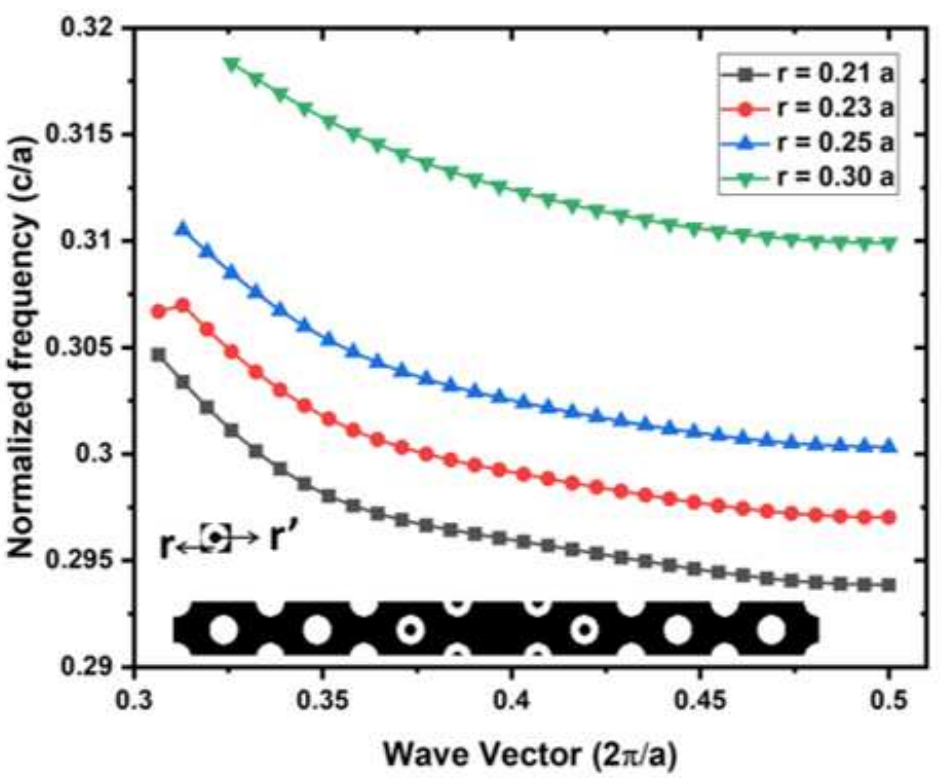

(b)

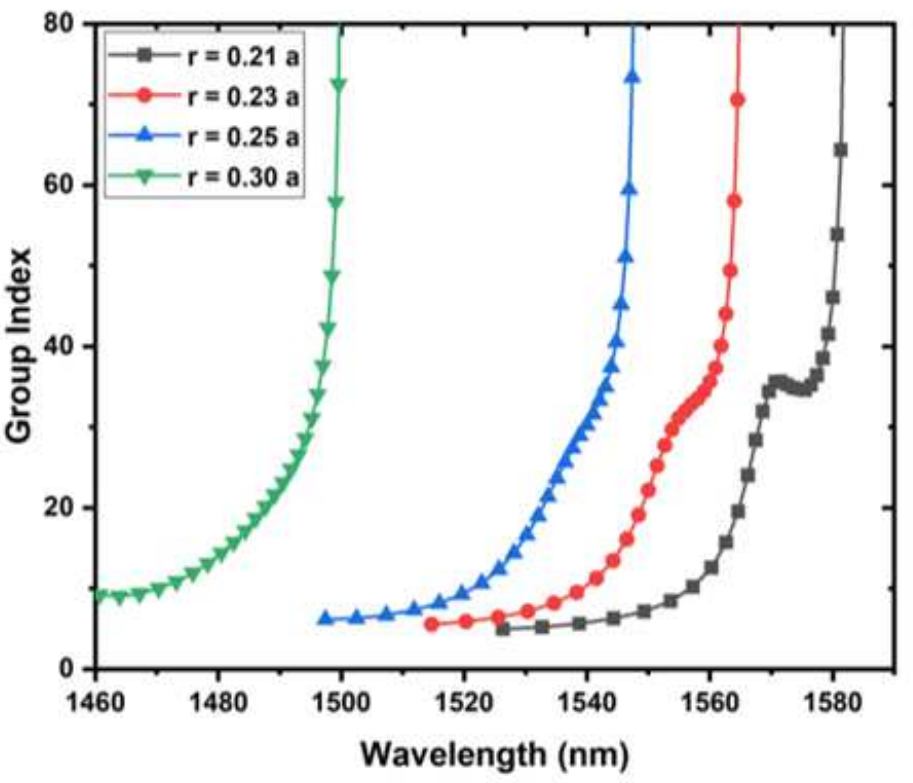

(d)

Figure 3

Band 15 from the band diagram of the PCW (a) with holes and (b) with rings. Group index plot of the PCW (c) with holes and (d) with rings. 


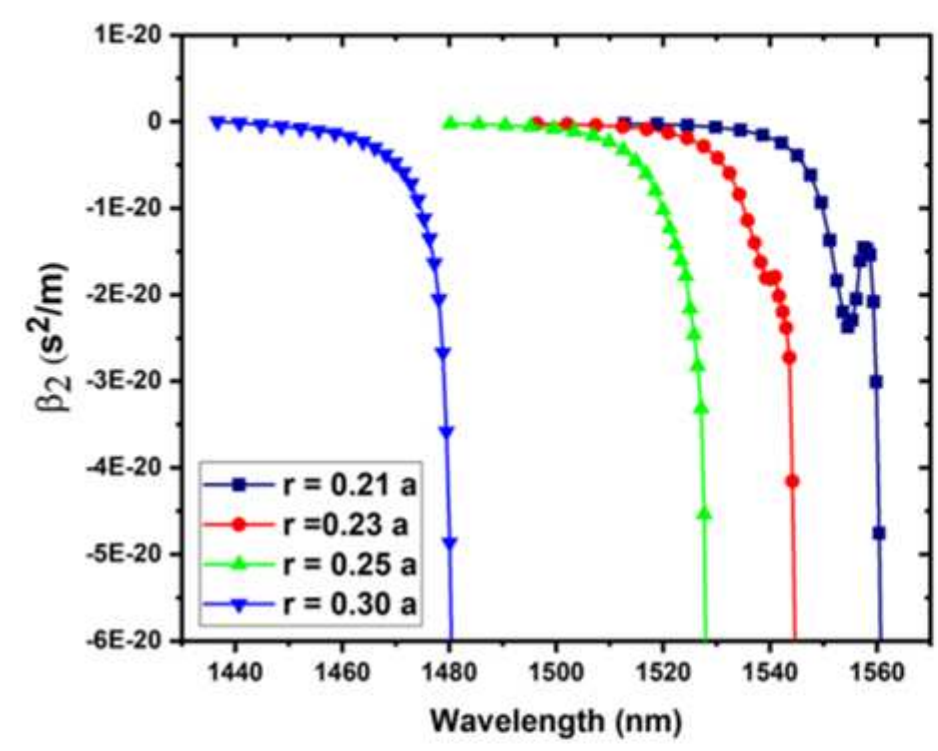

(a)

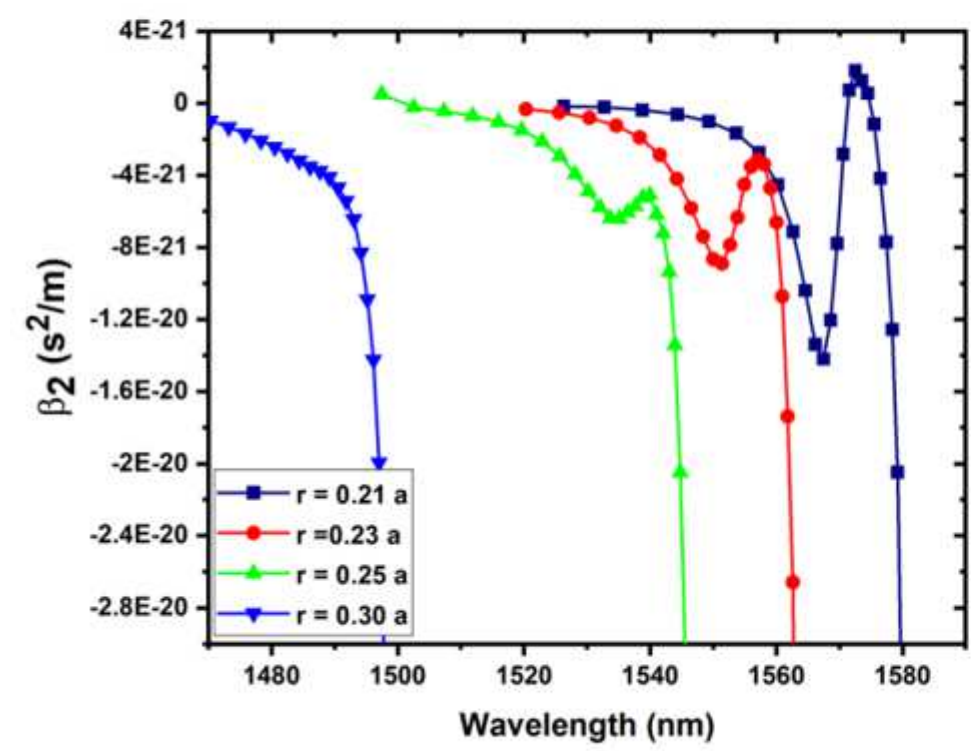

(c)

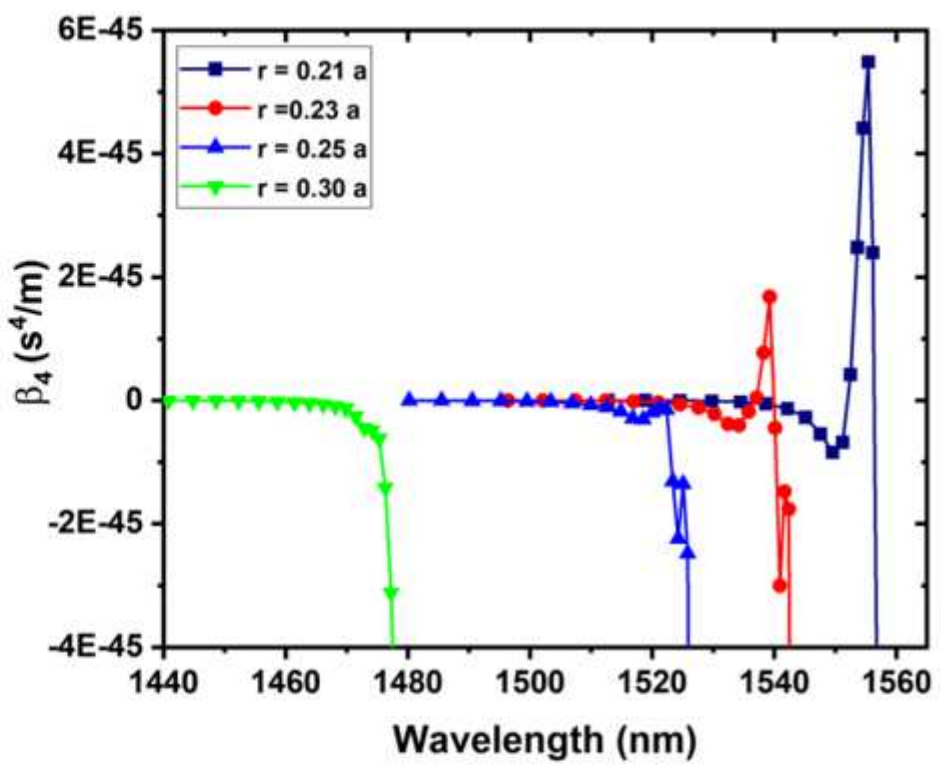

(b)

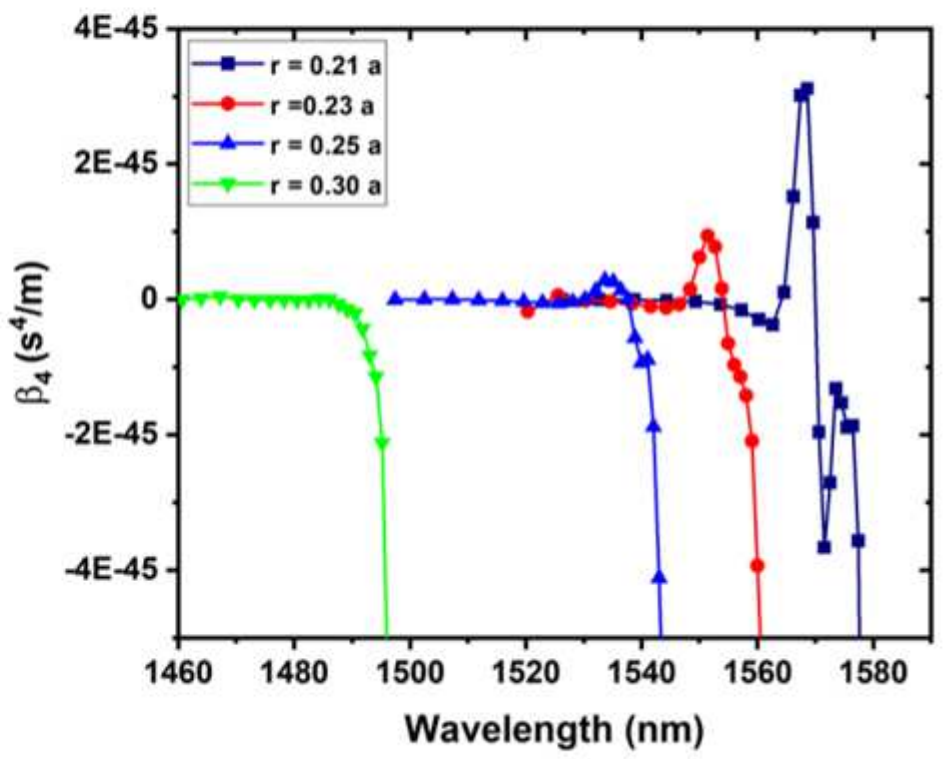

(d)

\section{Figure 4}

Dispersion characteristics corresponding to figure 3 (c-d). (a) $\beta 2$ of PCW with holes (b) $\beta 4$ of PCW with holes (c) $\beta 2$ of PCW with rings (d) $\beta 4$ of PCW with rings. 


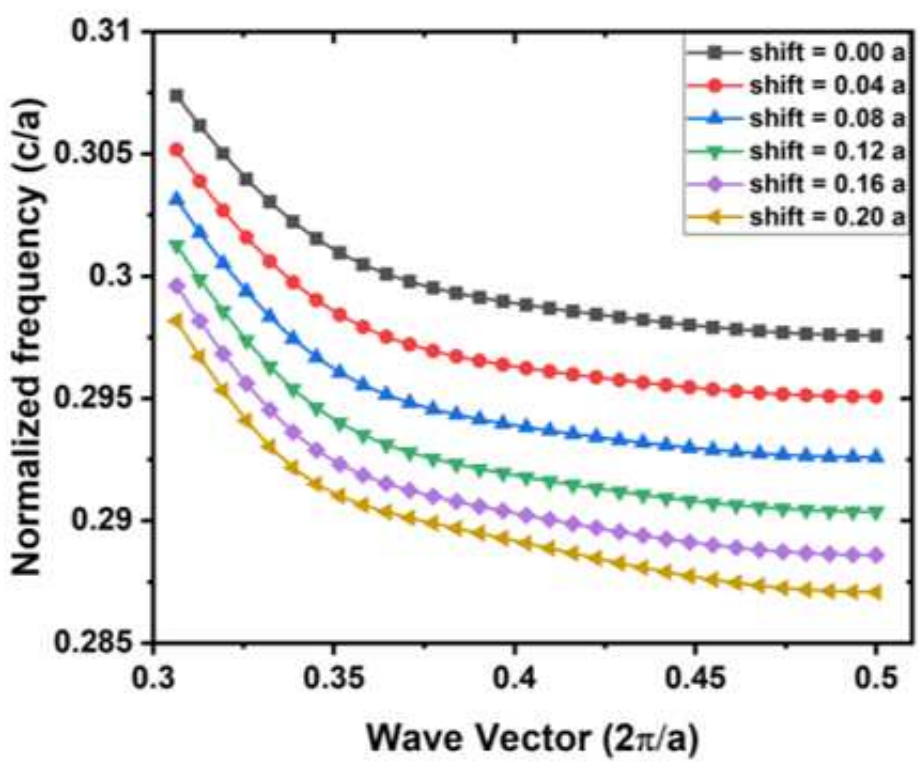

(a)

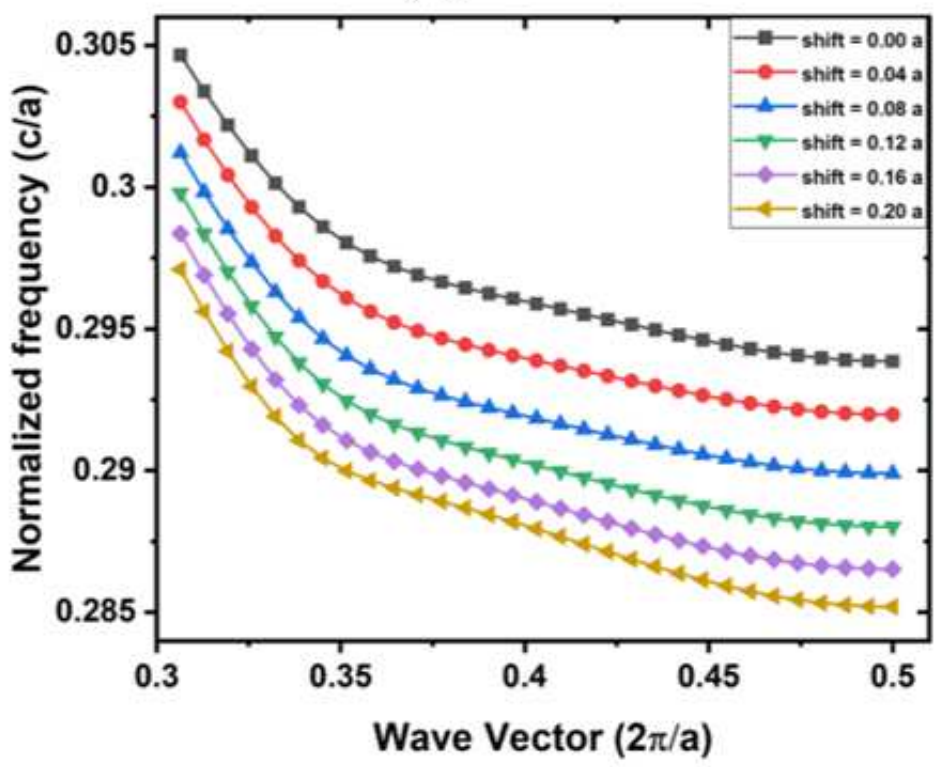

(c)

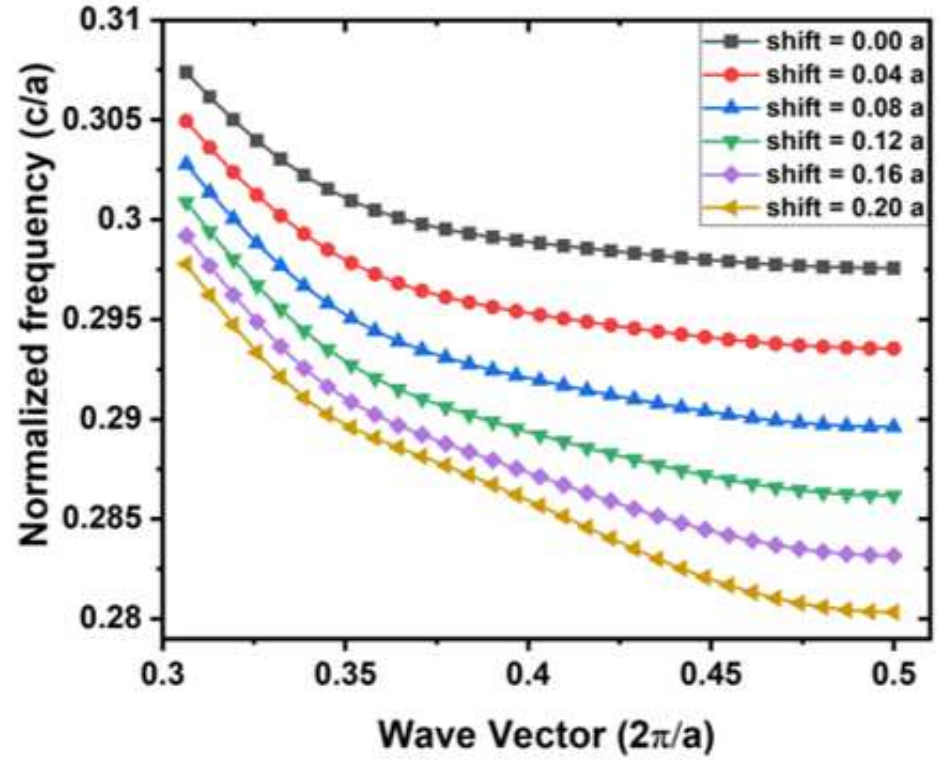

(b)

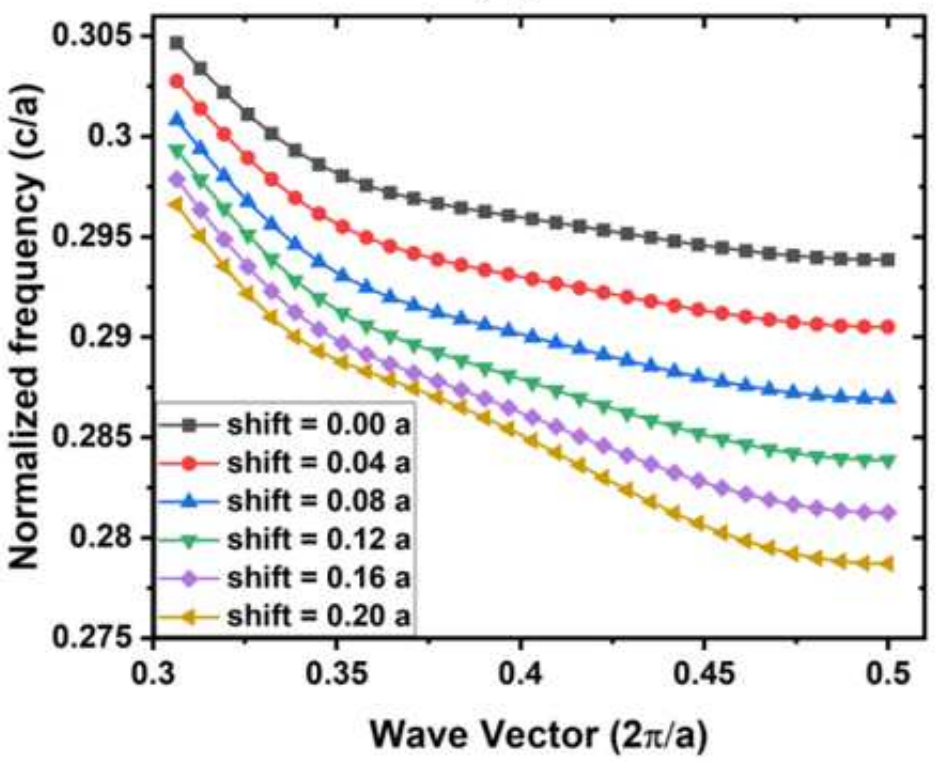

(d)

Figure 5

Band 15 from the band diagram of PCW with holes and (a) shift in one row (b) shift in two rows. Band 15 from the band diagram of PCW with rings and (c) shift in one row (d) shift in two rows. 


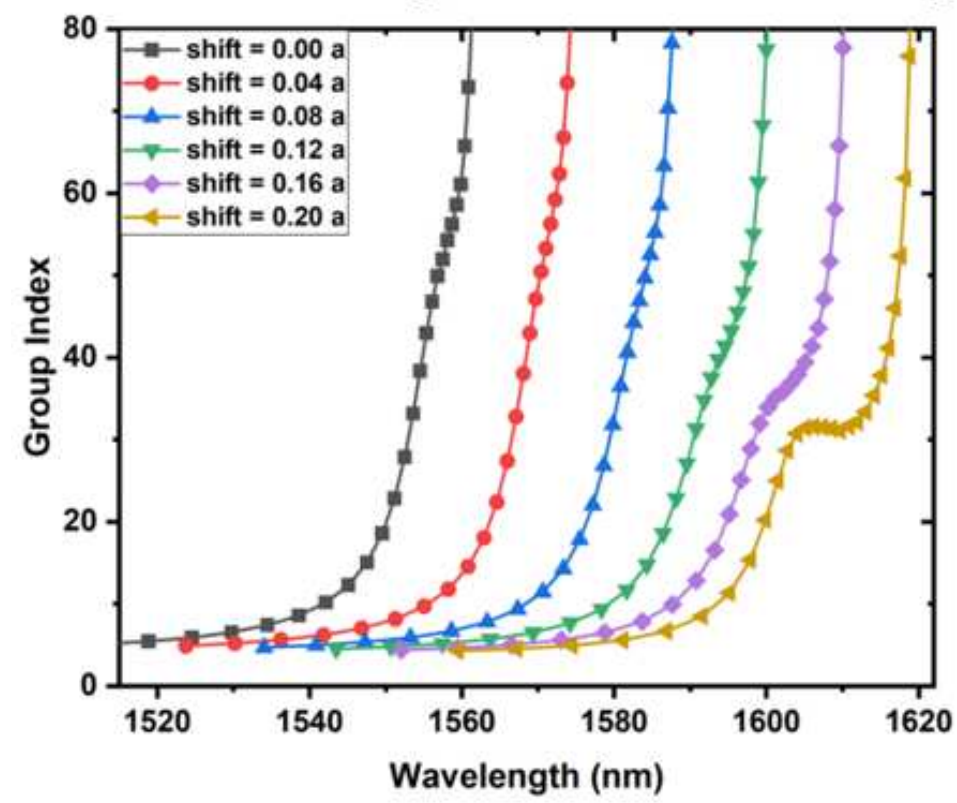

(a)

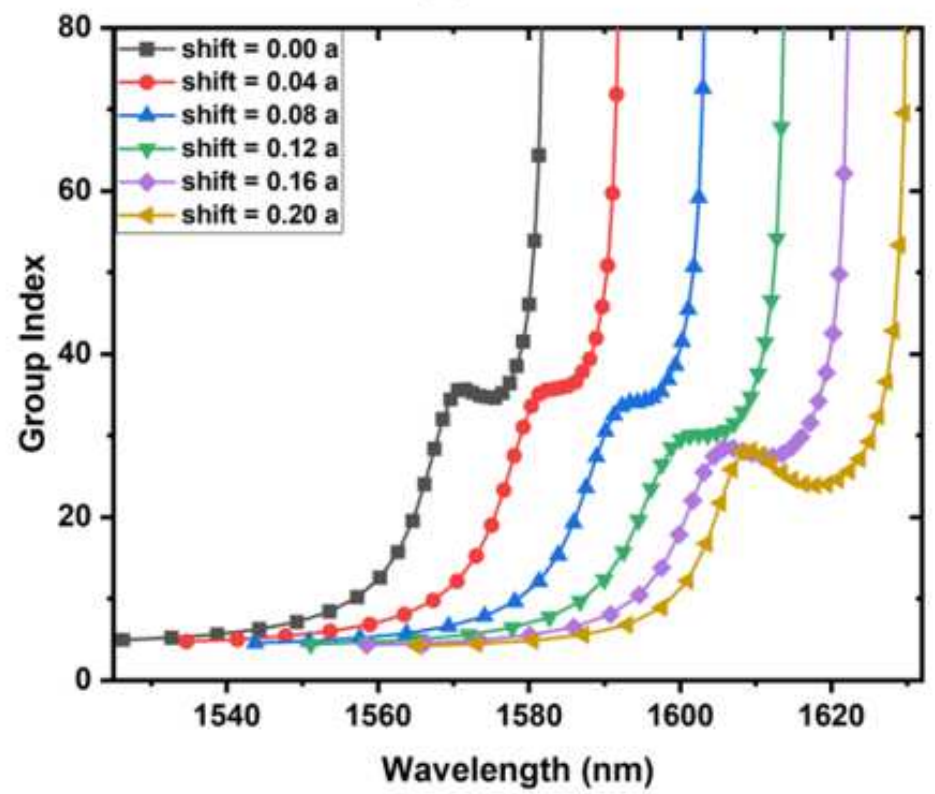

(c)

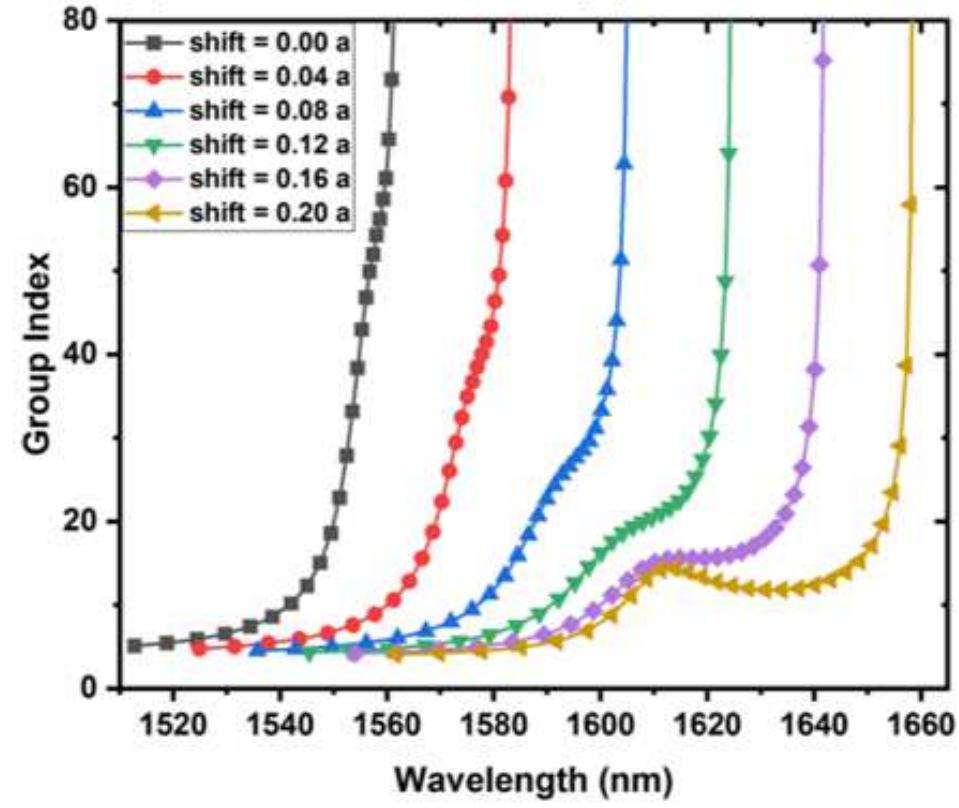

(b)

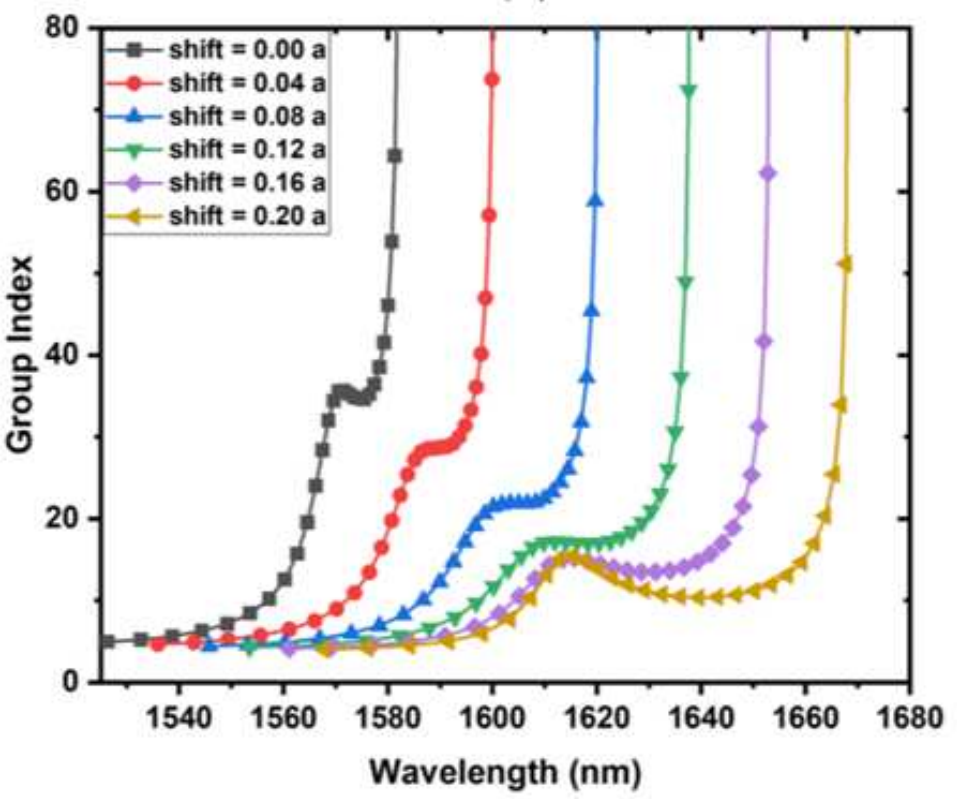

(d)

Figure 6

Group index - wavelength plot of the PCW with holes and (a) shift in one row (b) shift in two rows. Group index - wavelength plot of the PCW with rings and (c) shift in one row (d) shift in two rows. 


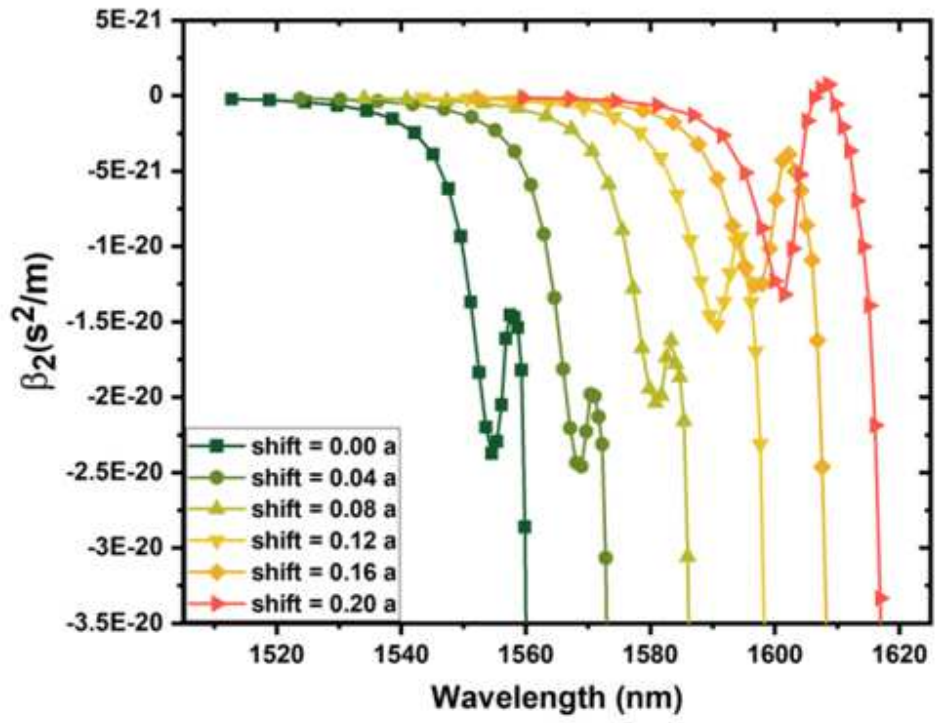

(a)

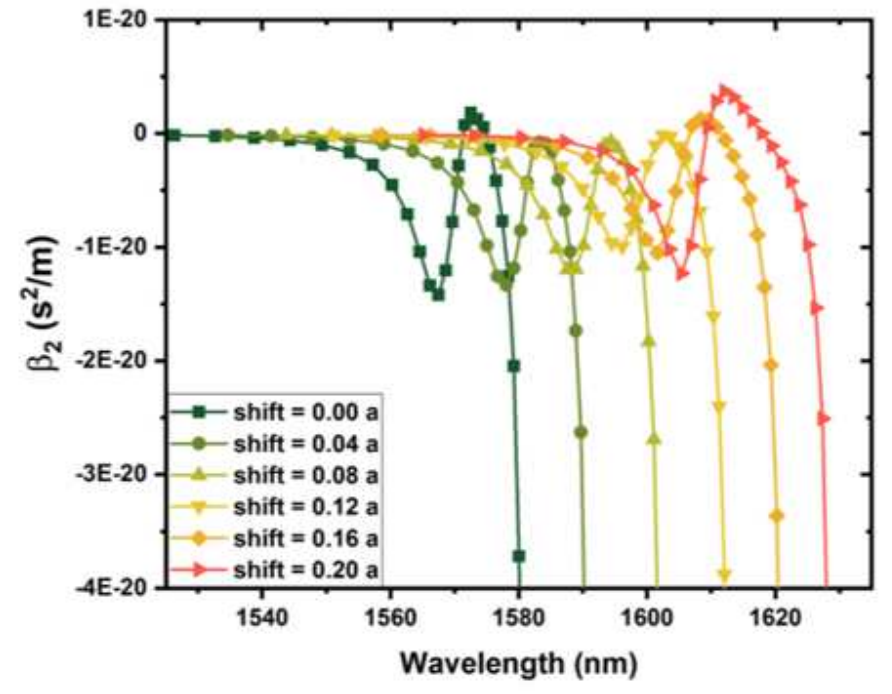

(c)

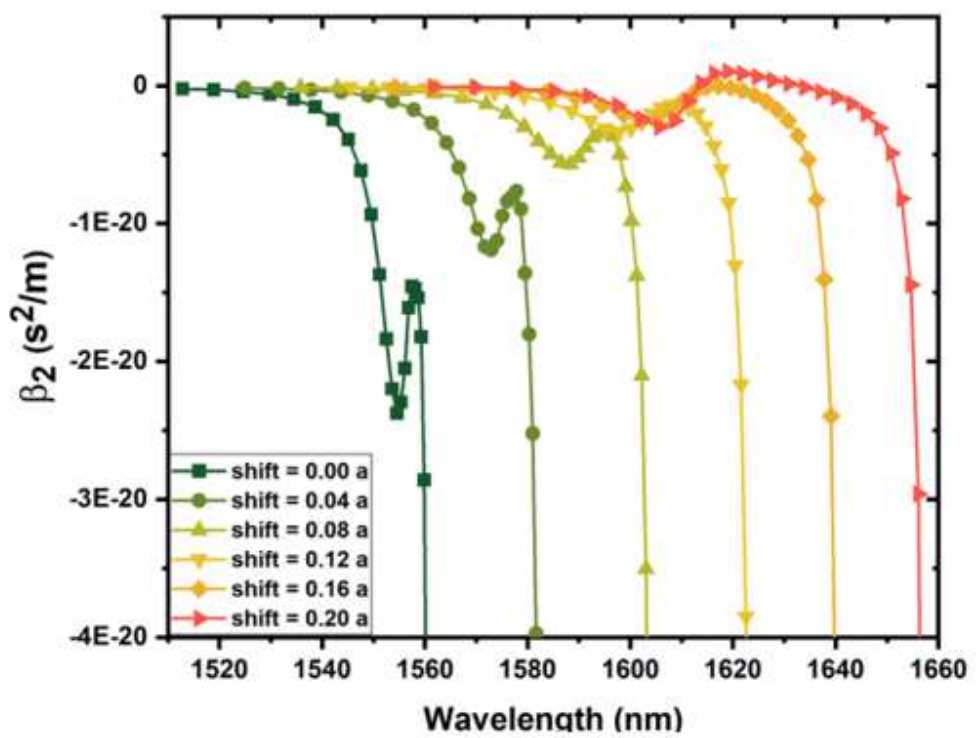

(b)

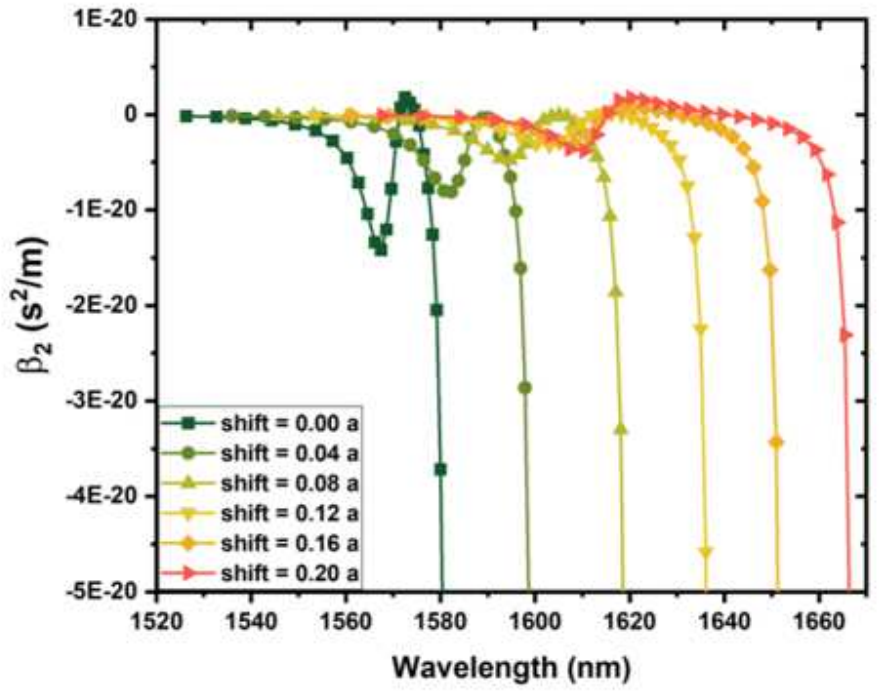

(d)

\section{Figure 7}

Dispersion characteristics corresponding to figure 6 (a-d). (a) $\beta 2$ of PCW with holes and shift in one row (b) $\beta 2$ of PCW with holes and shift in two rows (c) $\beta 2$ of PCW with rings and shift in one row (d) $\beta 2$ of PCW with rings and shift in two rows. 


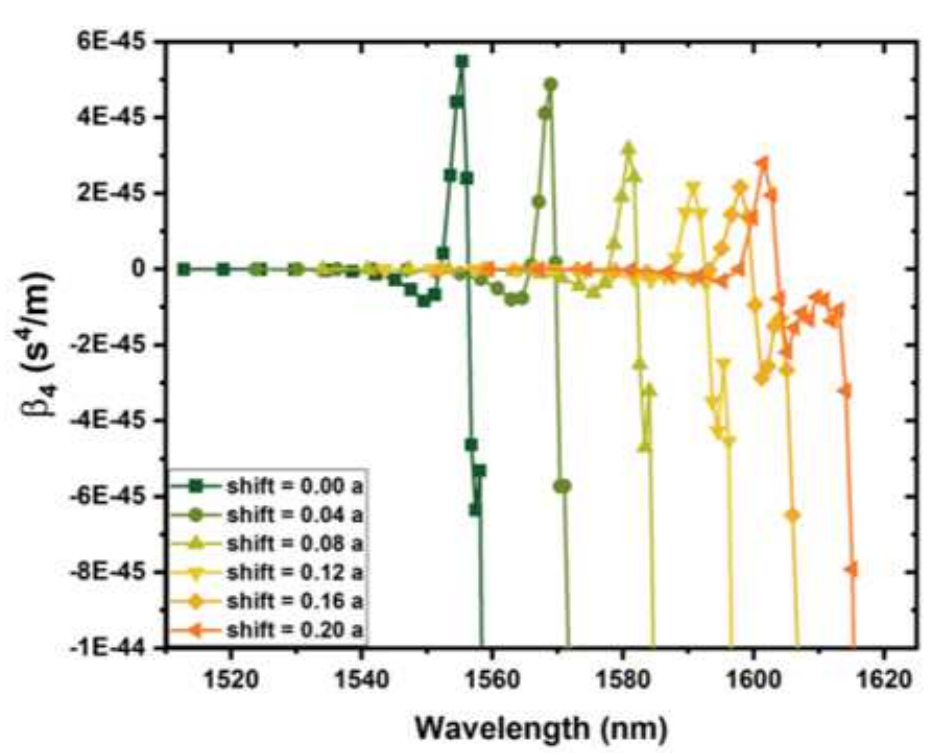

(a)

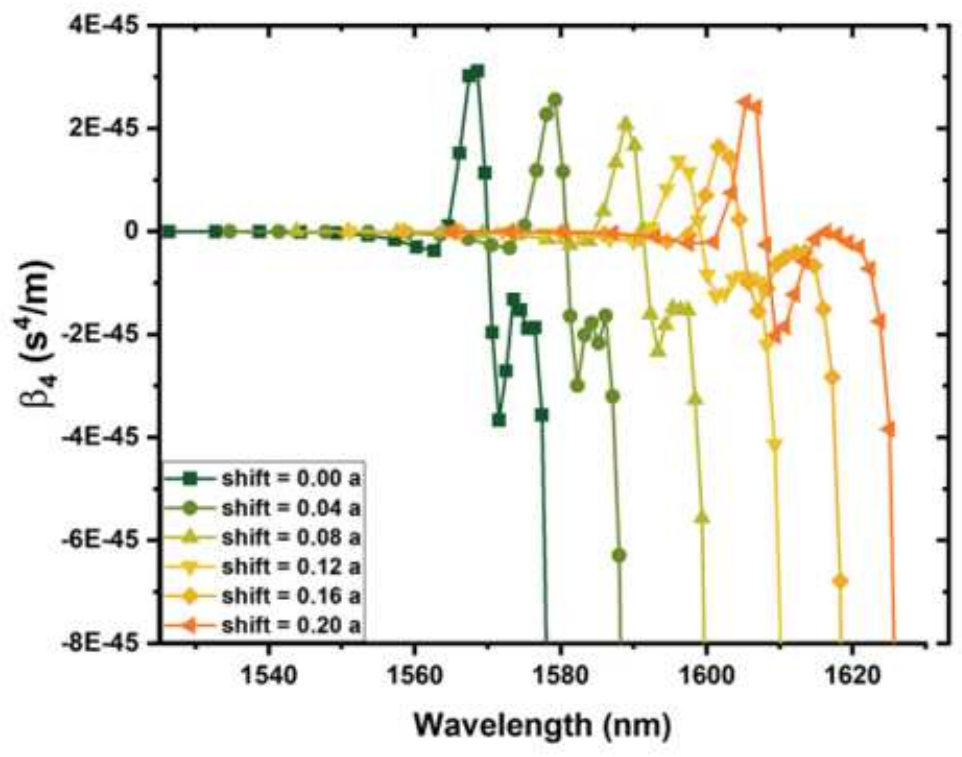

(c)

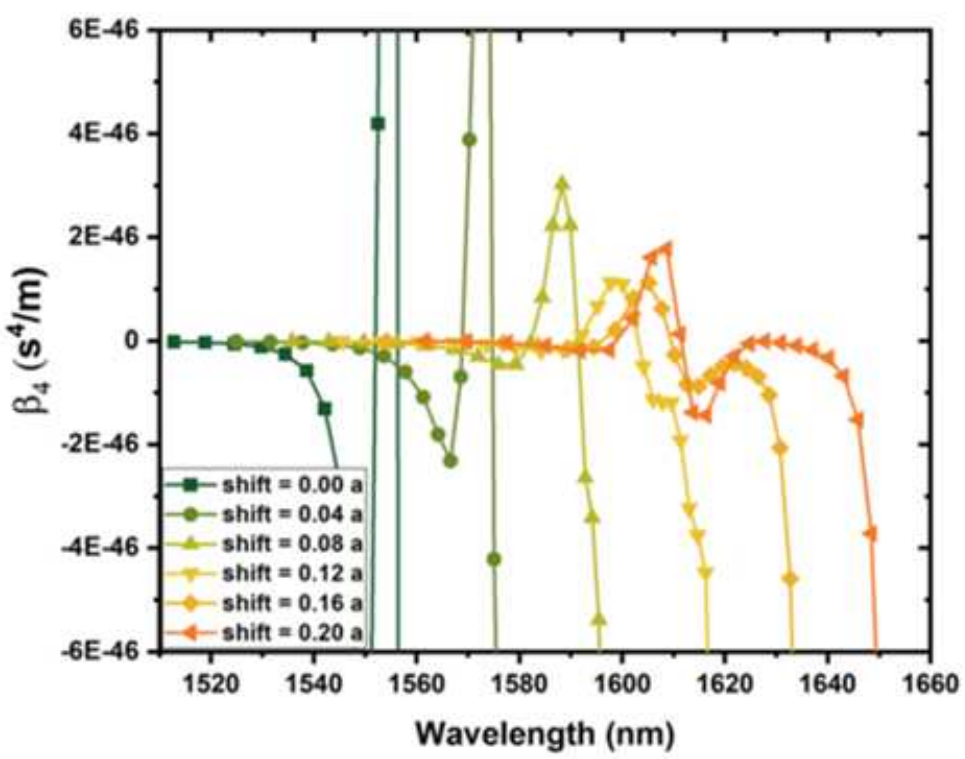

(b)

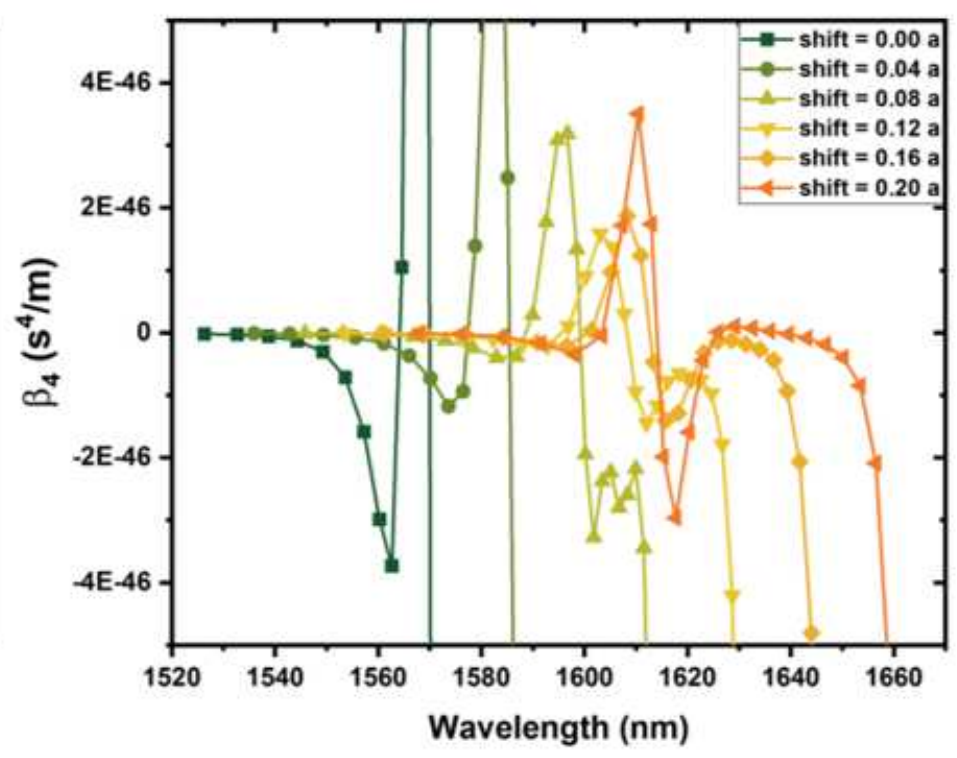

(d)

\section{Figure 8}

Dispersion characteristics corresponding to figure 6 (a-d). (a) $\beta 4$ of PCW with holes and shift in one row (b) $\beta 4$ of PCW with holes and shift in two rows (c) $\beta 4$ of PCW with rings and shift in one row (d) $\beta 4$ of PCW with rings and shift in two rows. 


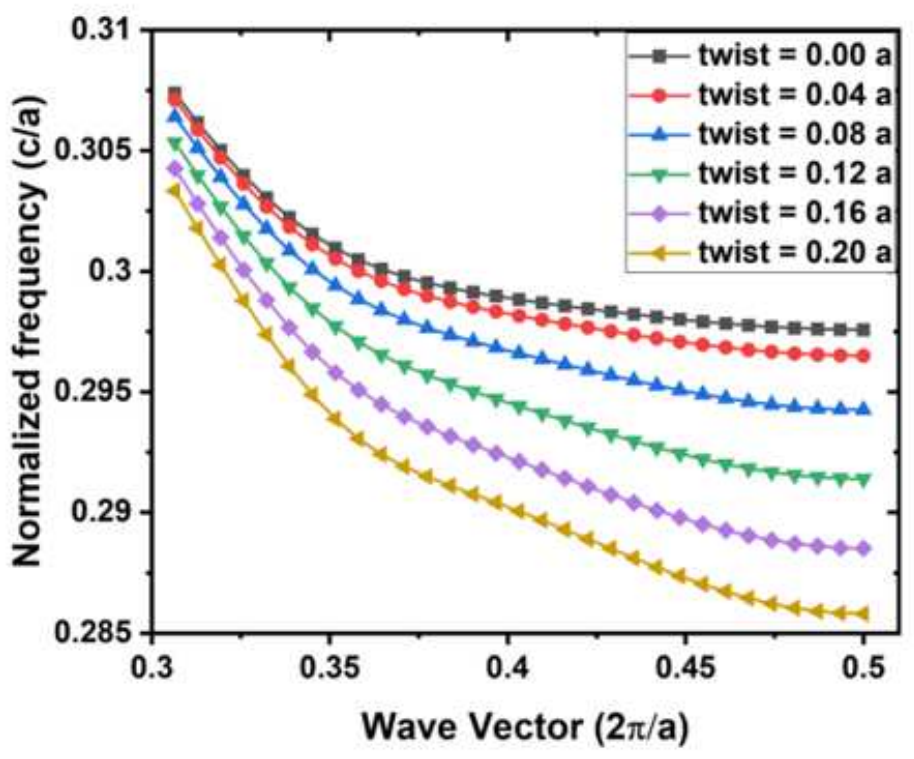

(a)

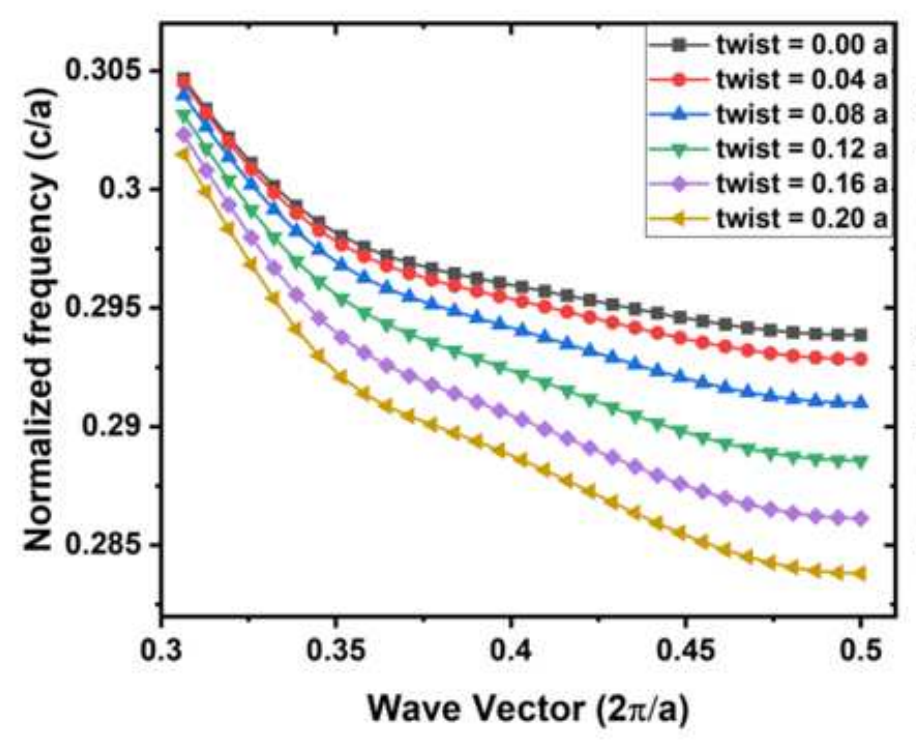

(c)

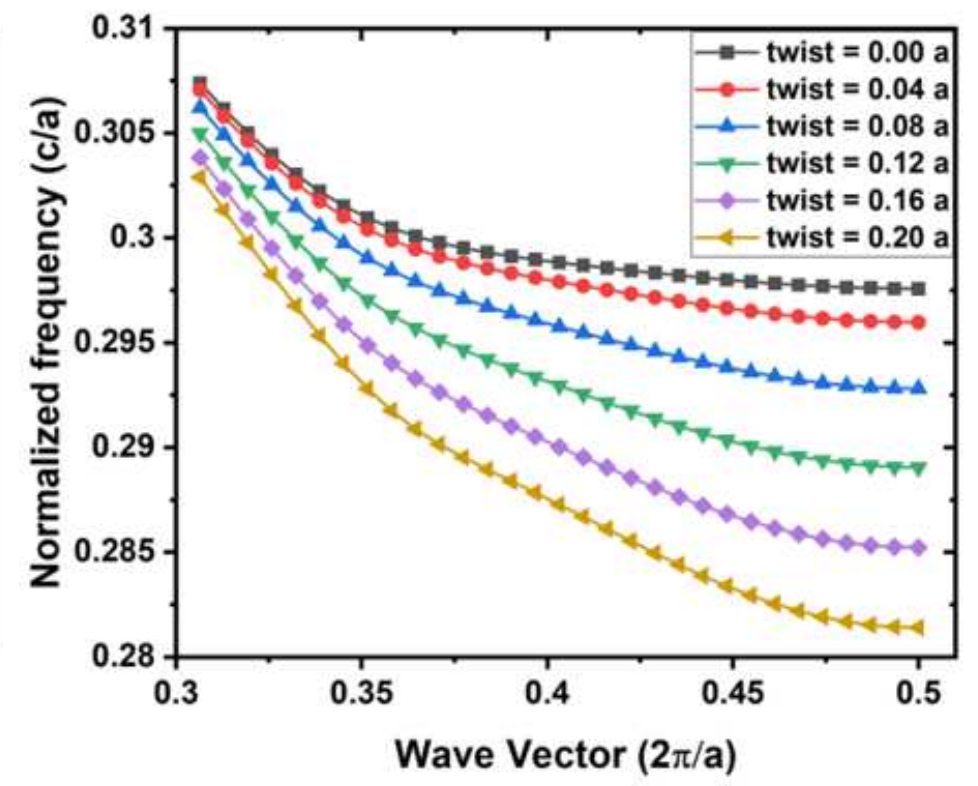

(b)

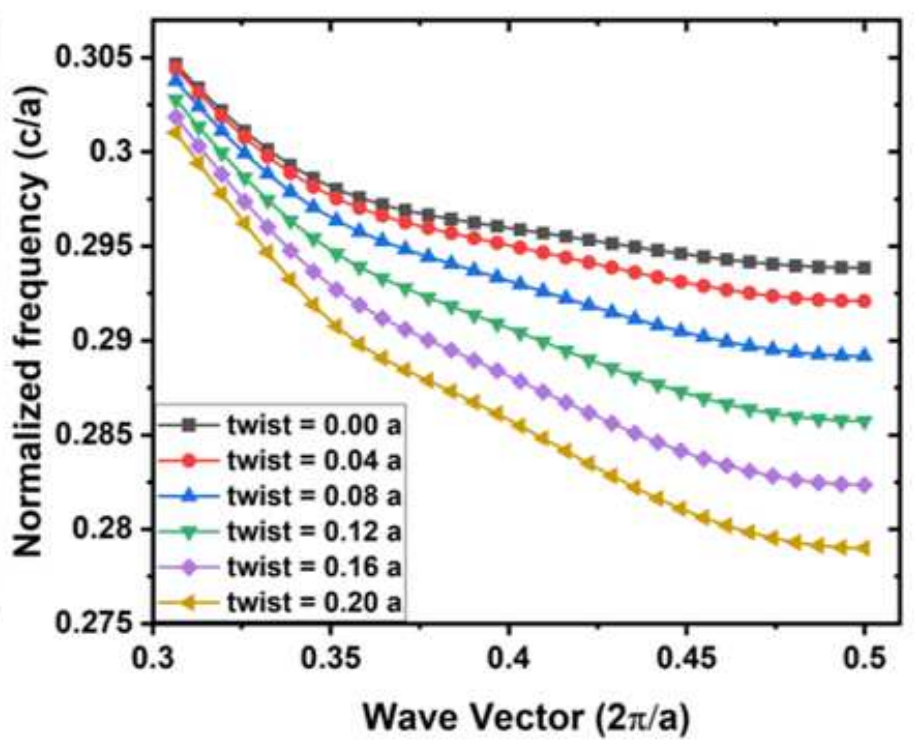

(d)

\section{Figure 9}

Band 15 from the band diagram of PCW with holes and (a) twist in one row (b) twist in two rows. Band 15 from the band diagram of PCW with rings and (c) twist in one row (d) twist in two rows. 


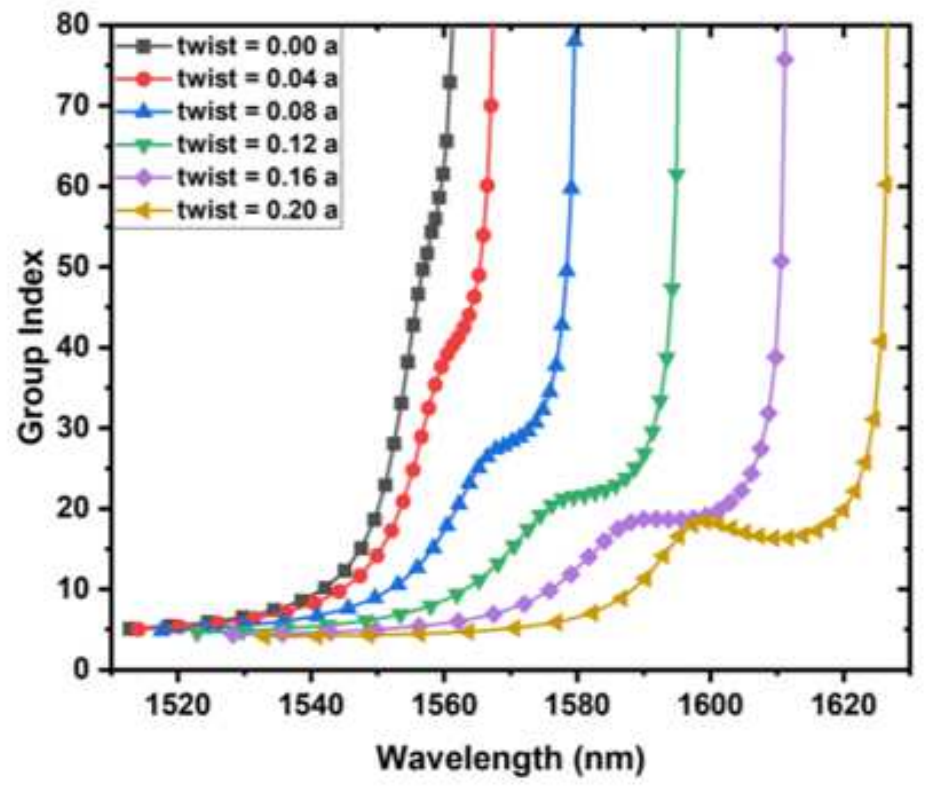

(a)

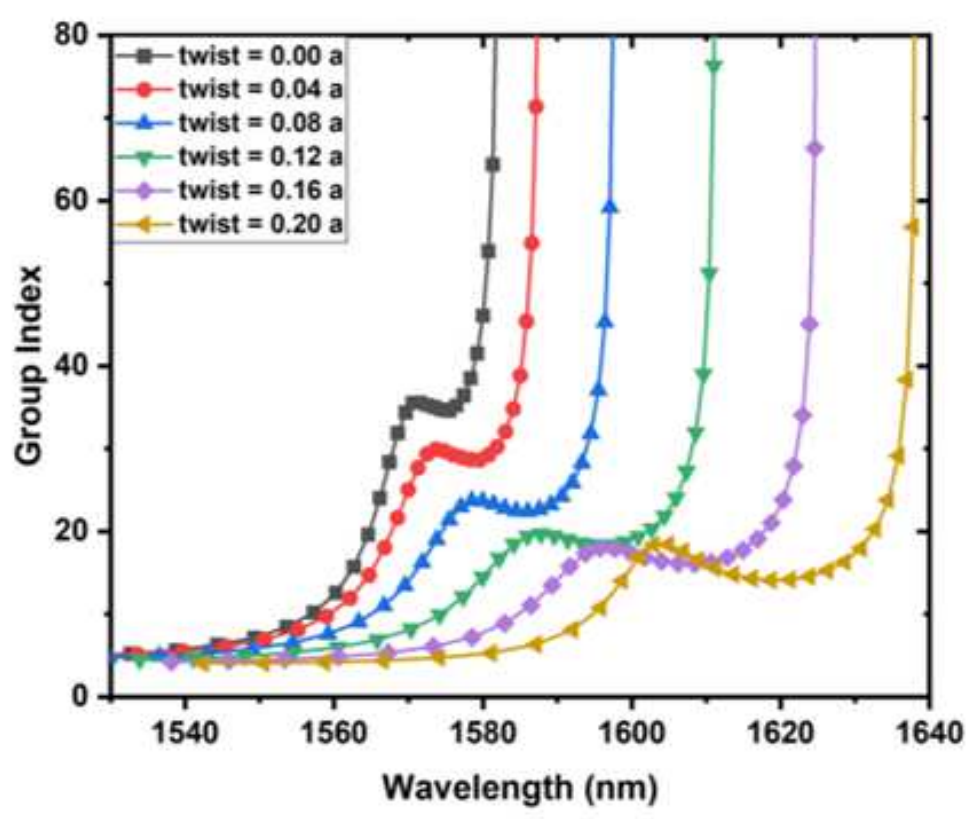

(c)

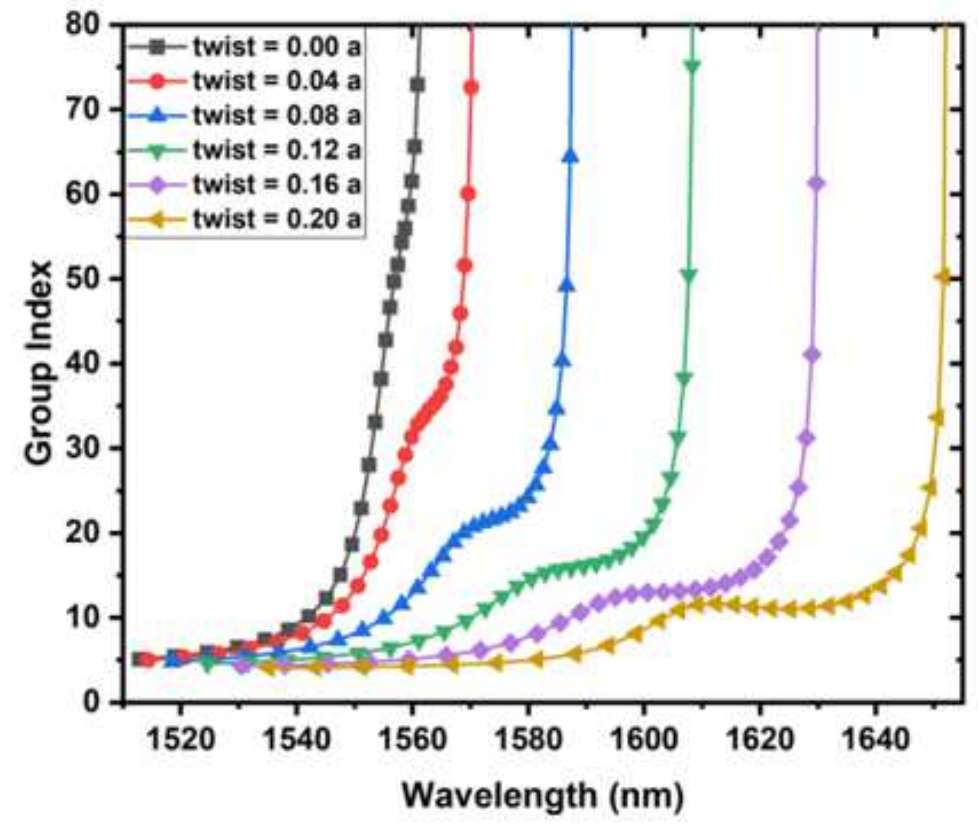

(b)

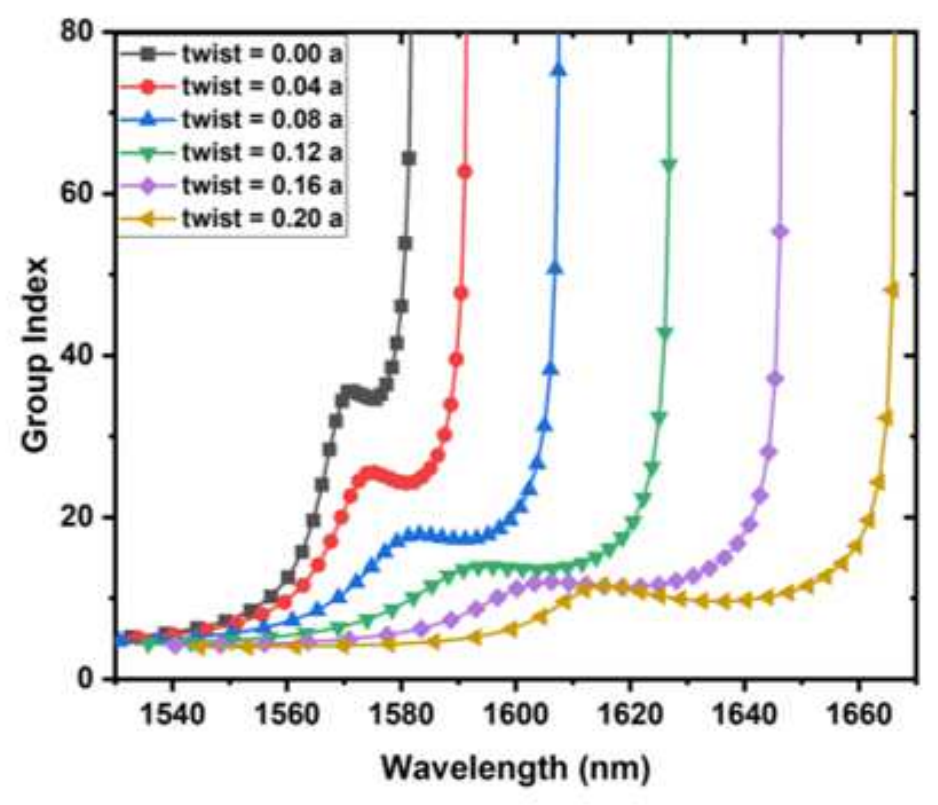

(d)

\section{Figure 10}

Group index - wavelength plot of the PCW with holes and (a) twist in one row (b) twist in two rows. Group index - wavelength plot of the PCW with rings and (c) twist in one row (d) twist in two rows. 


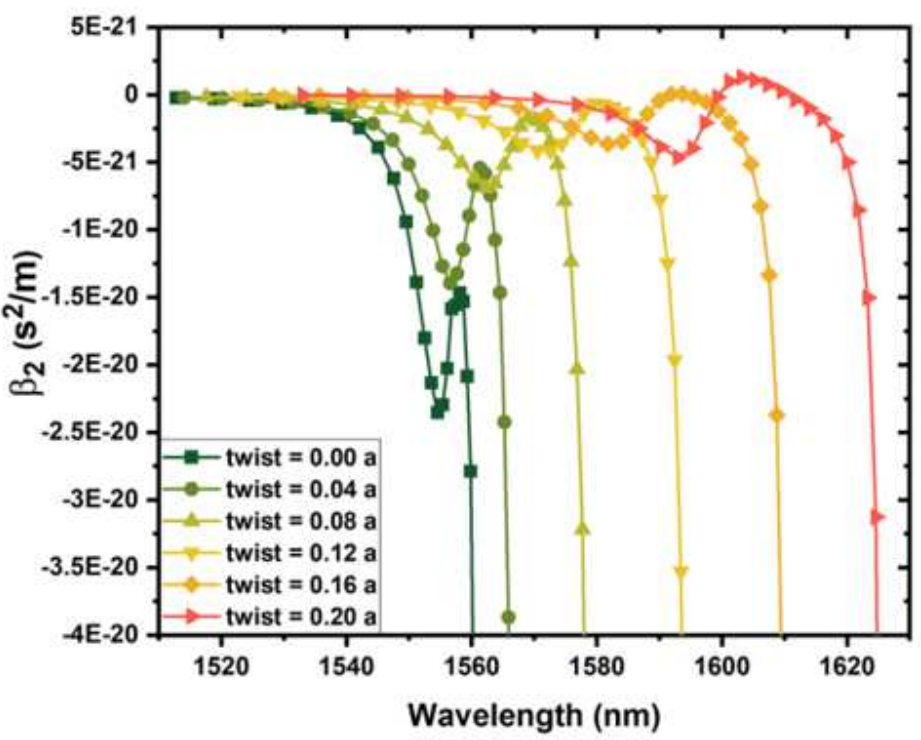

(a)

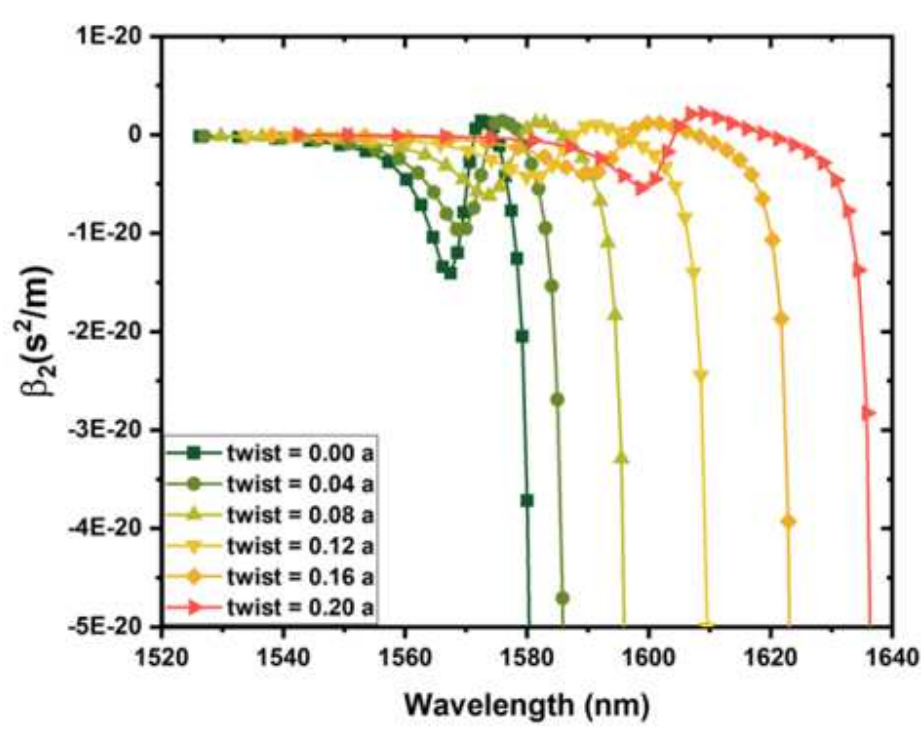

(c)

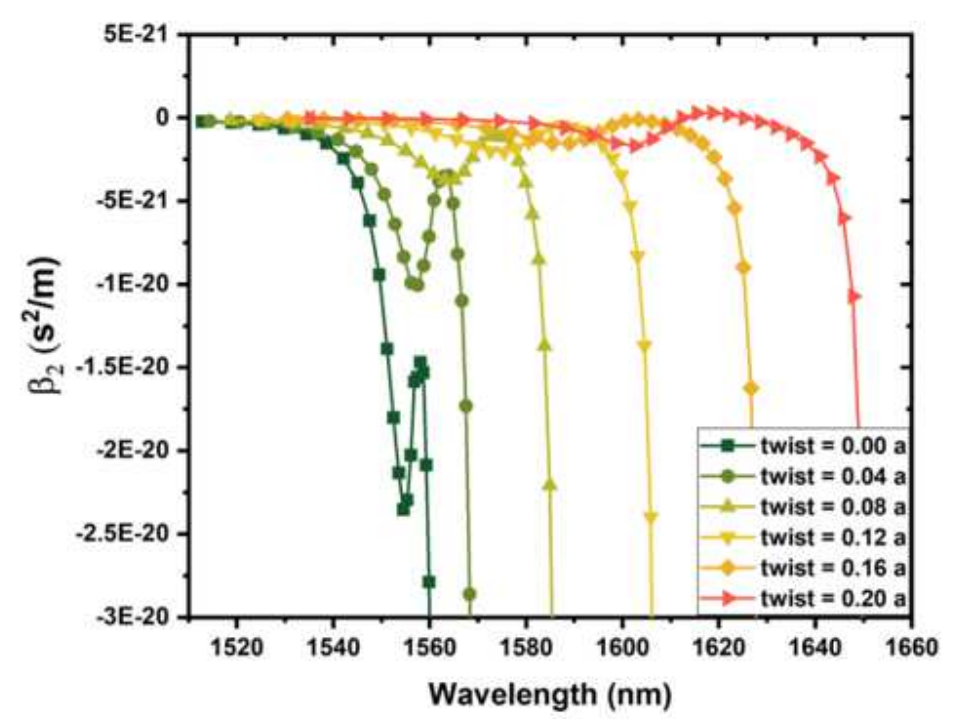

(b)

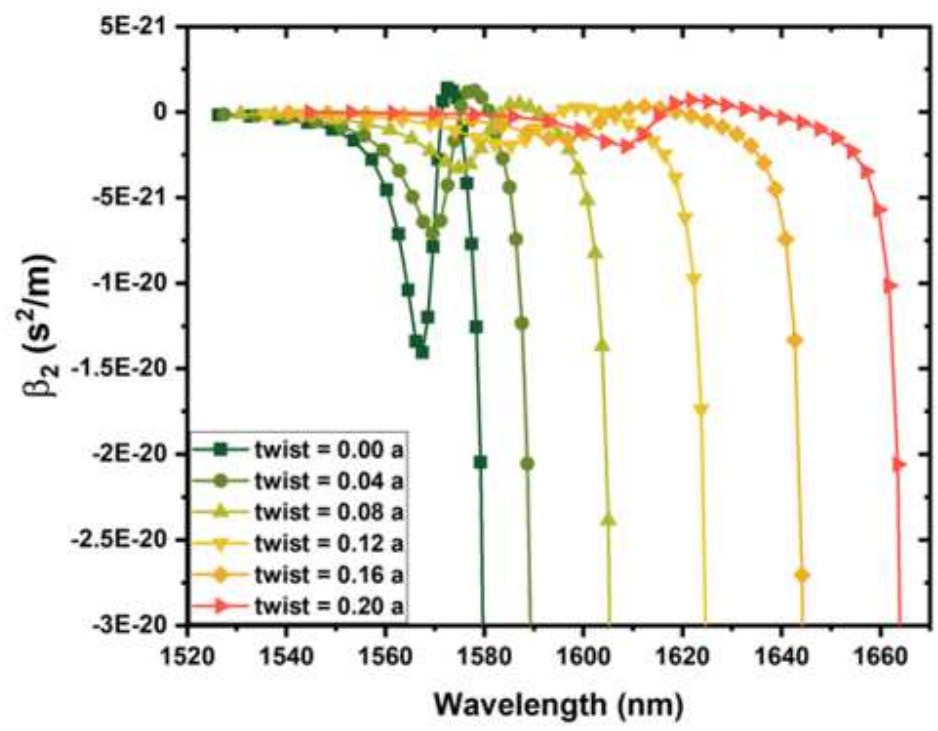

(d)

\section{Figure 11}

Dispersion characteristics corresponding to figure 10 (a-d). (a) $\beta 2$ of PCW with holes and twist in one row (b) $\beta 2$ of PCW with holes and twist in two rows (c) $\beta 2$ of PCW with rings and twist in one row (d) $\beta 2$ of PCW with rings and twist in two rows. 


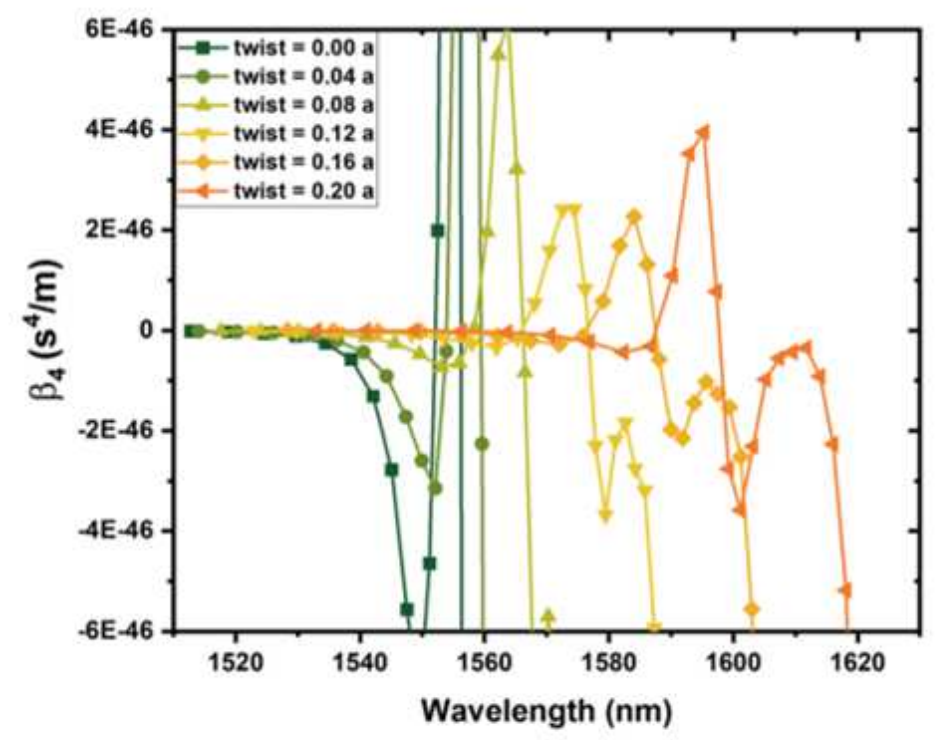

(a)

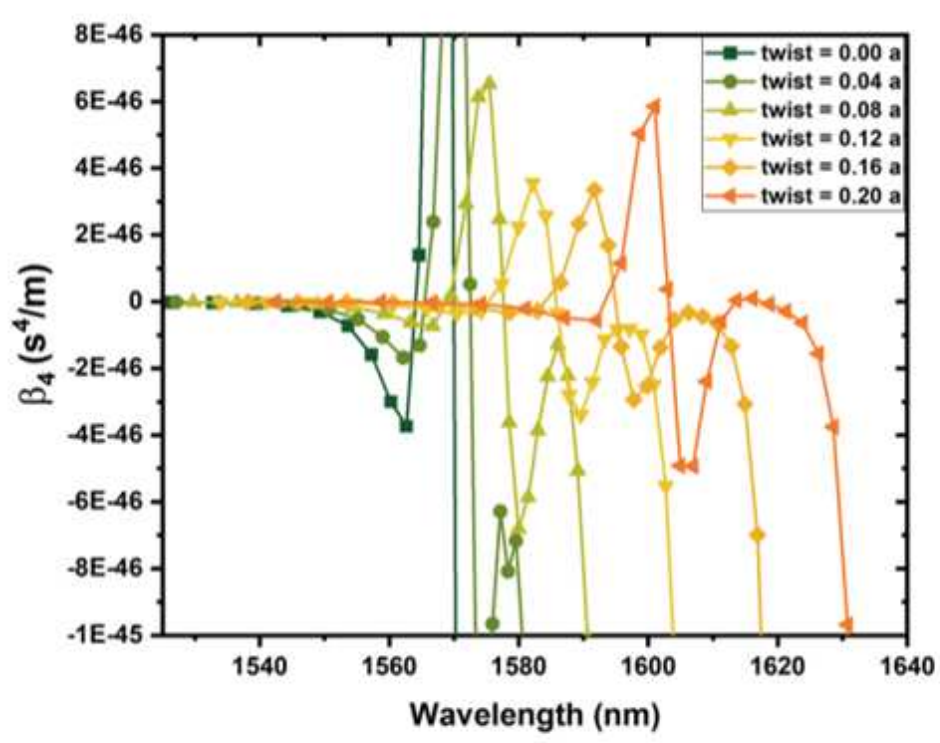

(c)

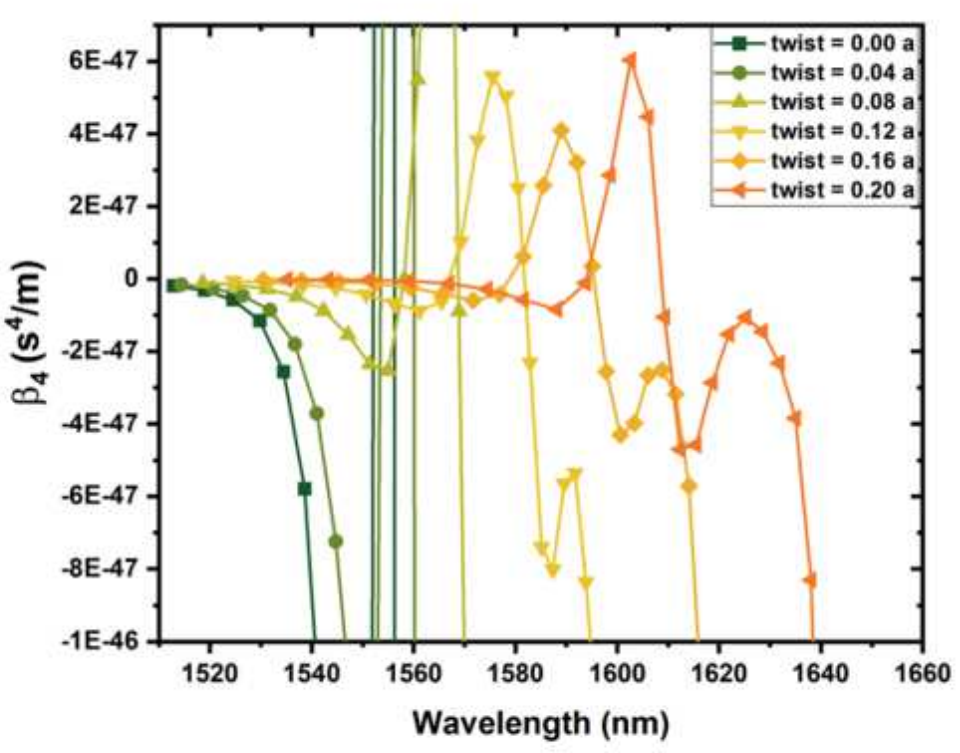

(b)

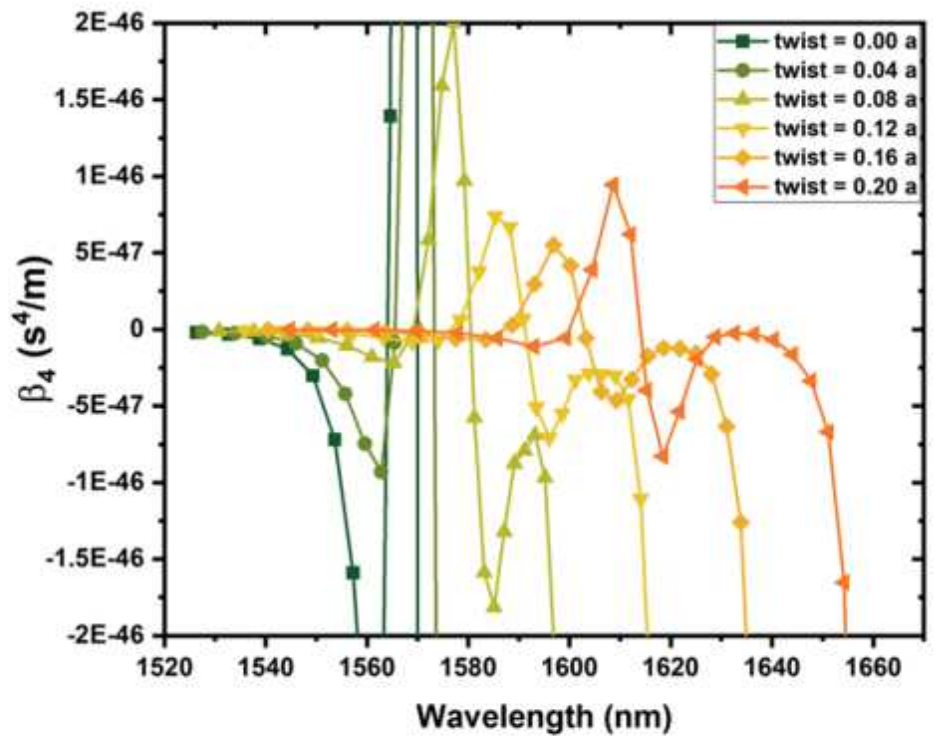

(d)

Figure 12

Dispersion characteristics corresponding to figure 10 (a-d). (a) $\beta 4$ of PCW with holes and twist in one row (b) $\beta 4$ of PCW with holes and twist in two rows (c) $\beta 4$ of PCW with rings and twist in one row (d) $\beta 4$ of PCW with rings and twist in two rows. 


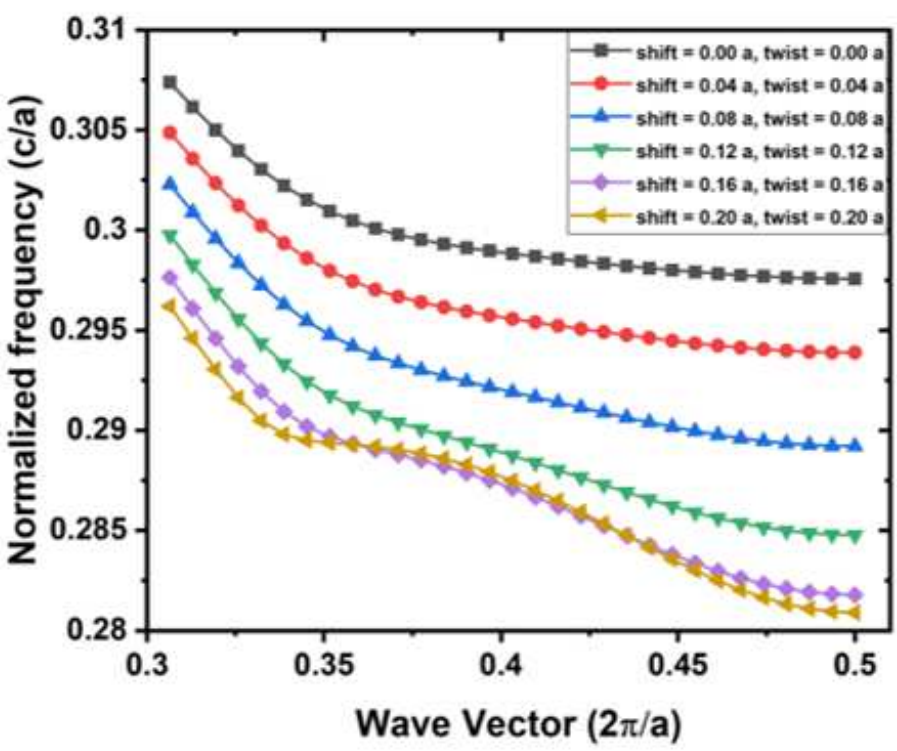

(a)

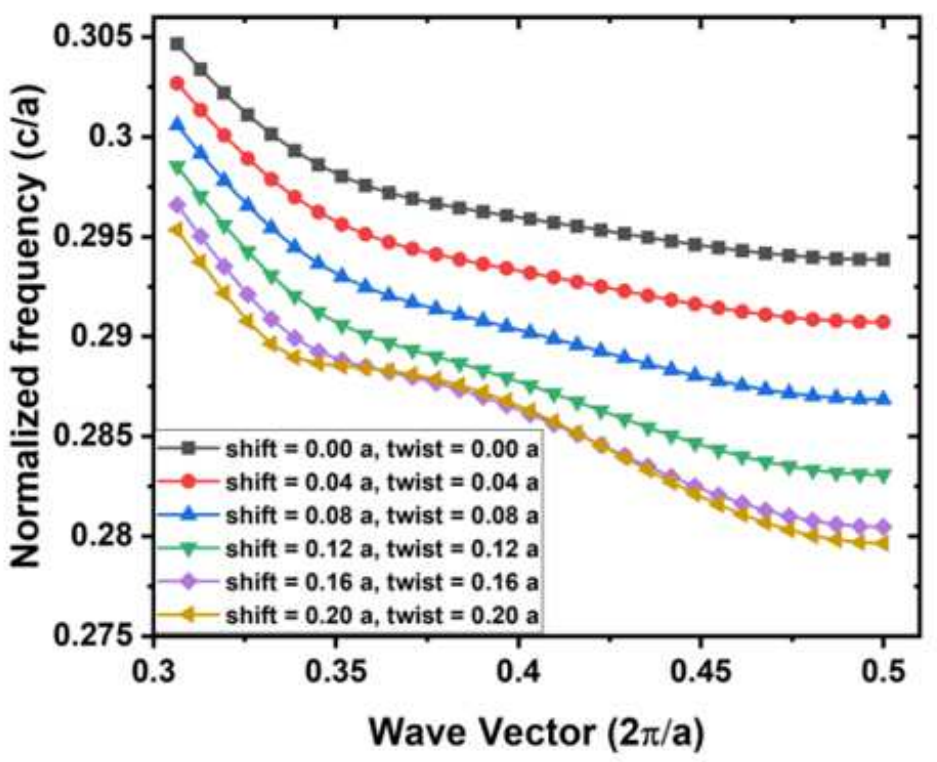

(c)

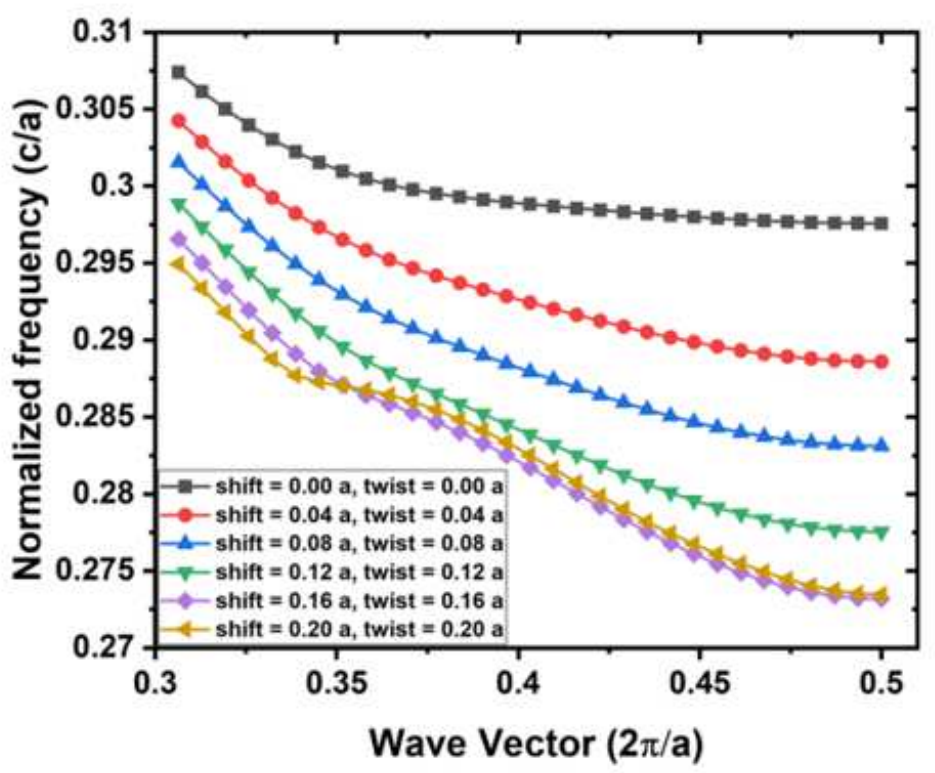

(b)

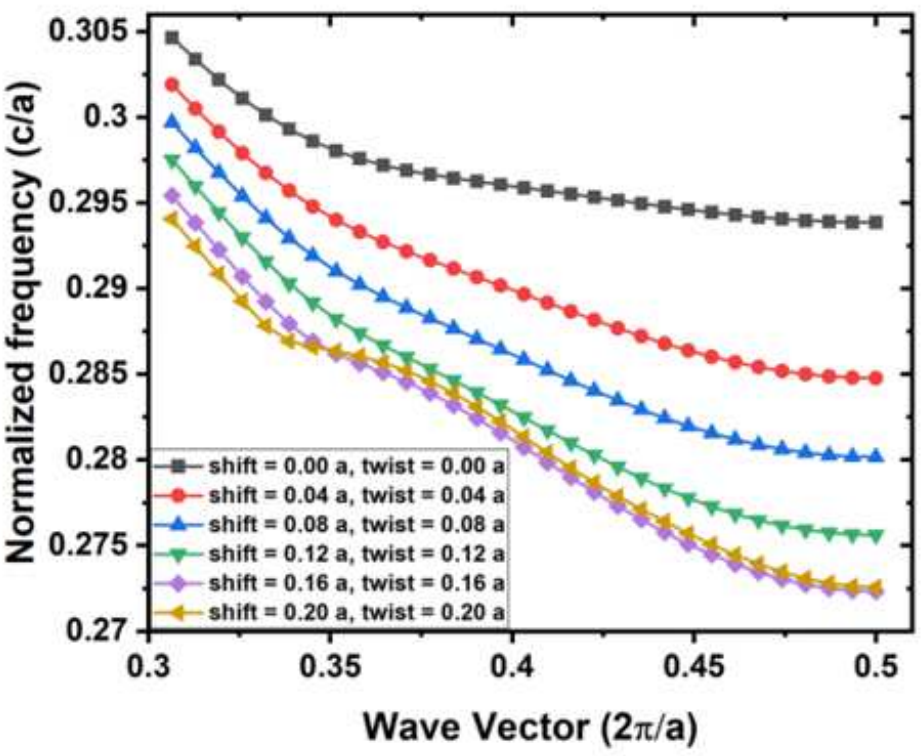

(d)

\section{Figure 13}

Group index - wavelength plot of the PCW with holes and applied (a) shift and twist in one row (b) shift and twist in two rows. Group index - wavelength plot of the PCW with rings and applied (c) shift and twist in one row (d) shift and twist in two rows. 


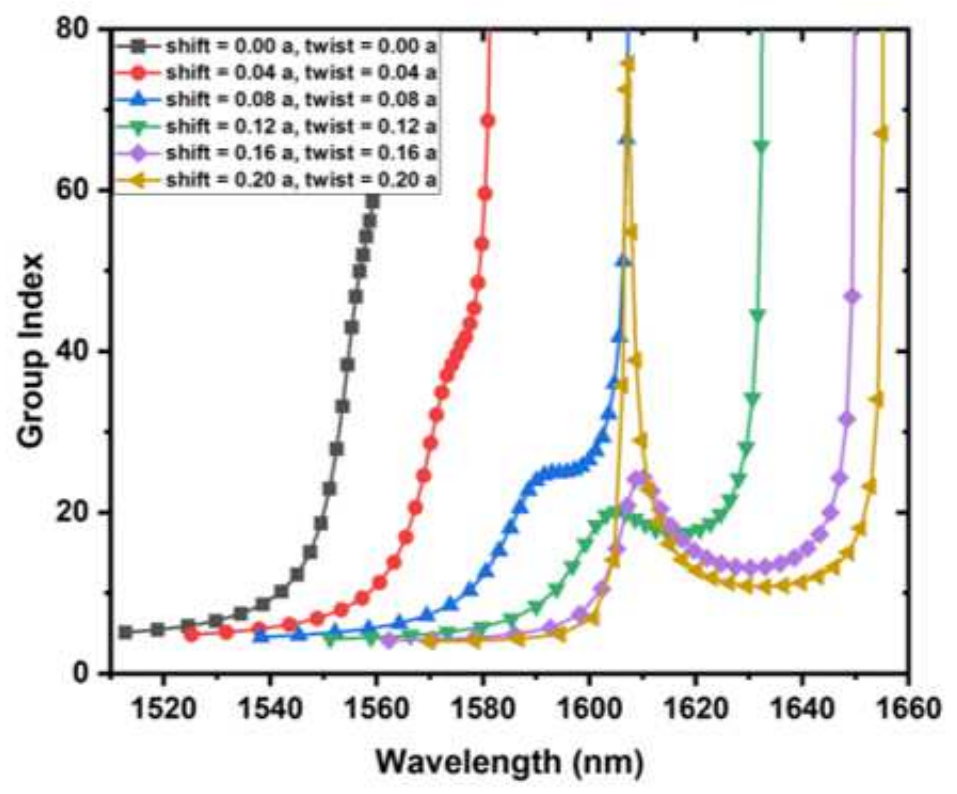

(a)

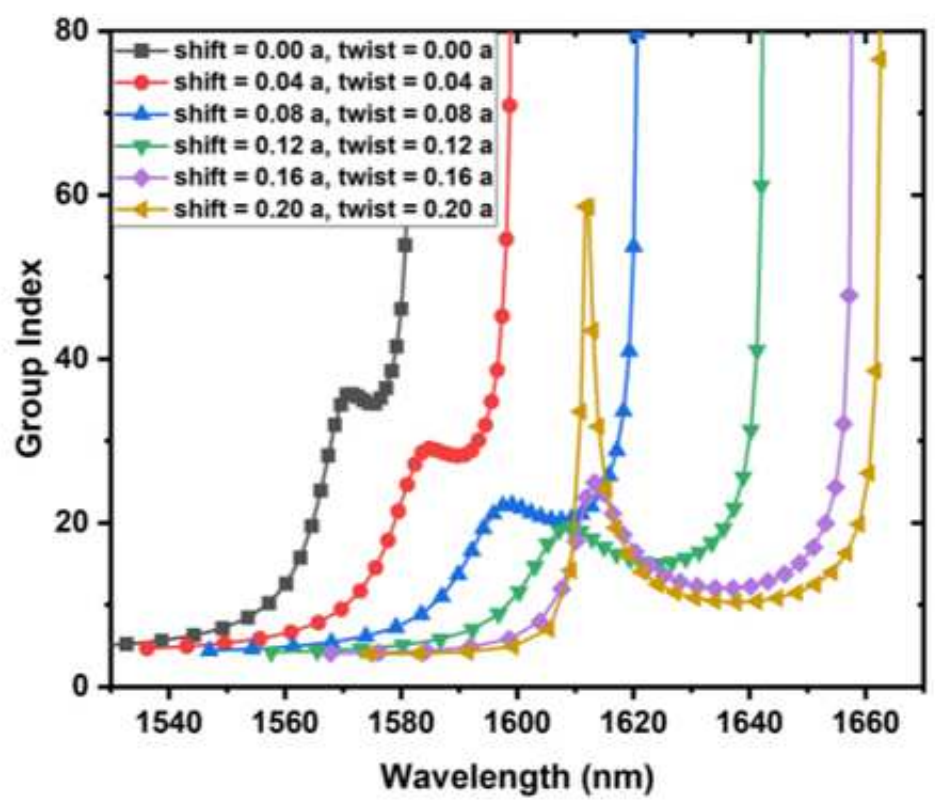

(c)

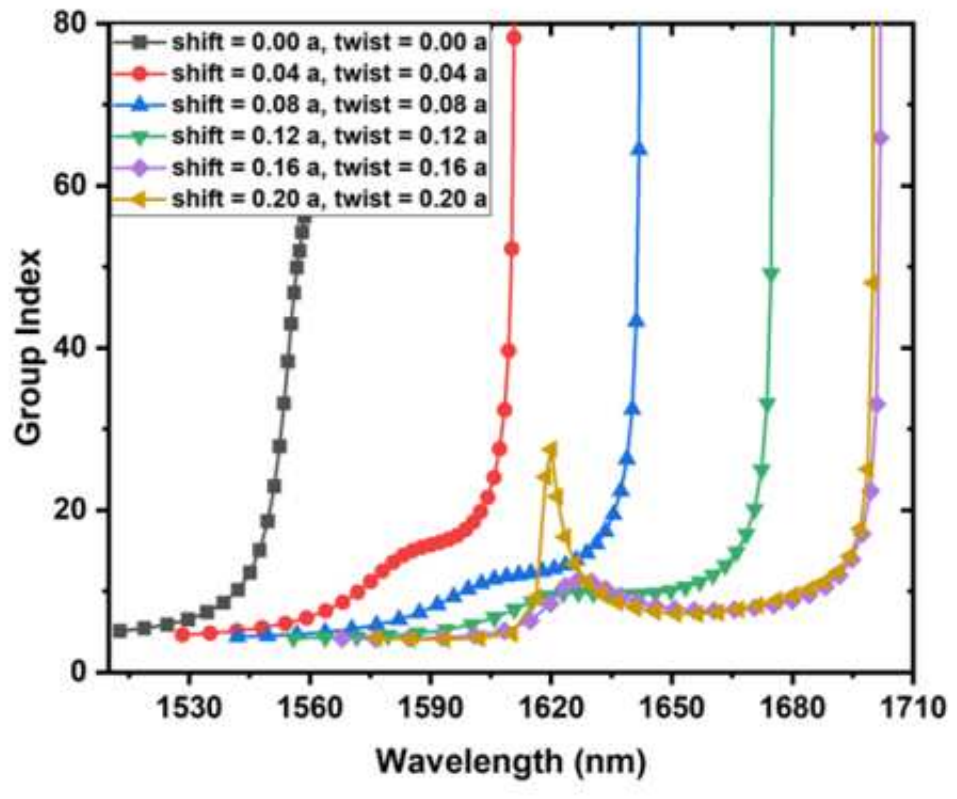

(b)

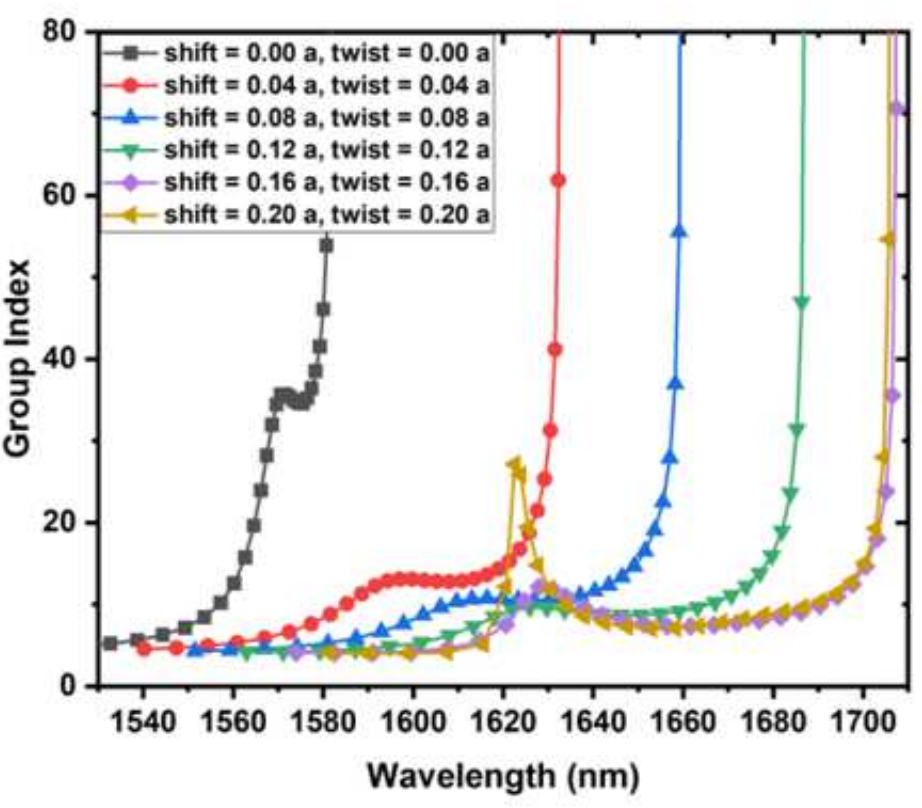

(d)

\section{Figure 14}

Group index - wavelength plot of the PCW with holes and applied (a) shift and twist in one row (b) shift and twist in two rows. Group index - wavelength plot of the PCW with rings and applied (c) shift and twist in one row (d) shift and twist in two rows. 


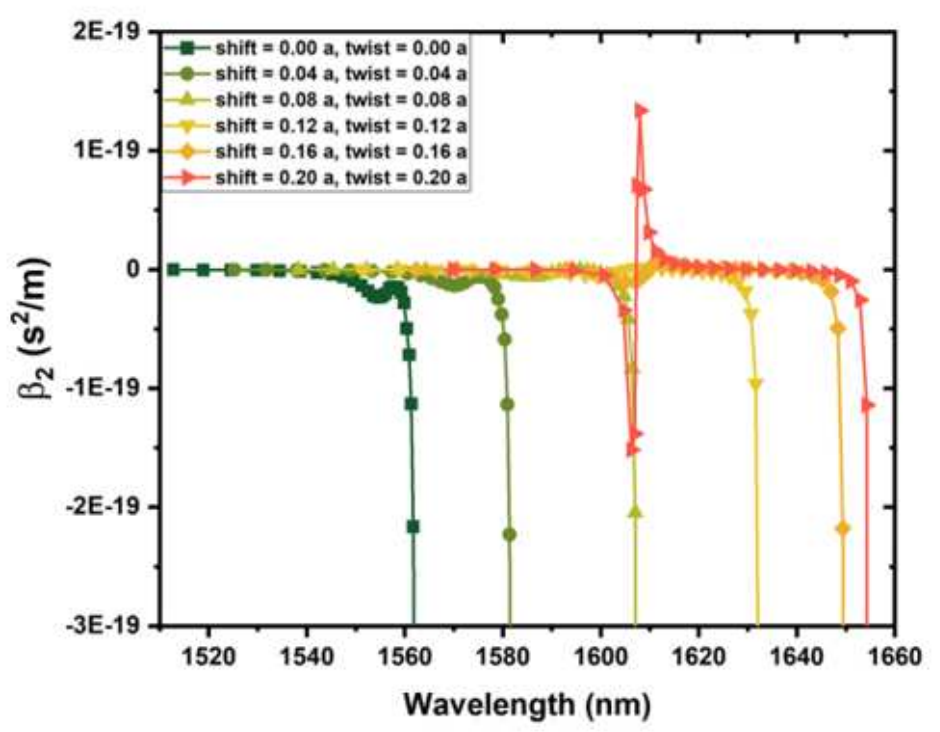

(a)

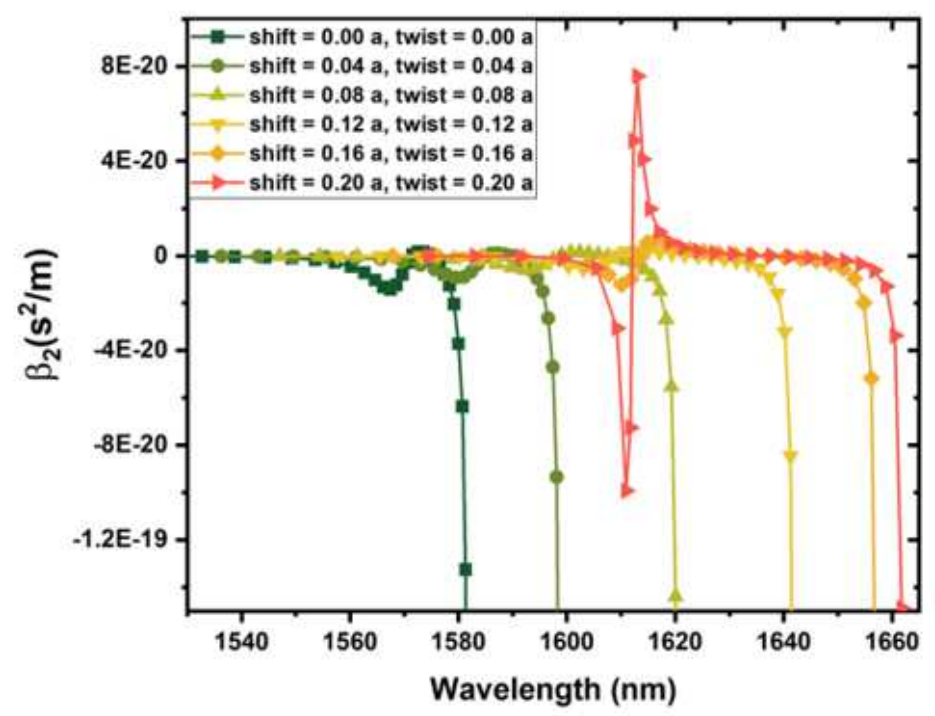

(c)

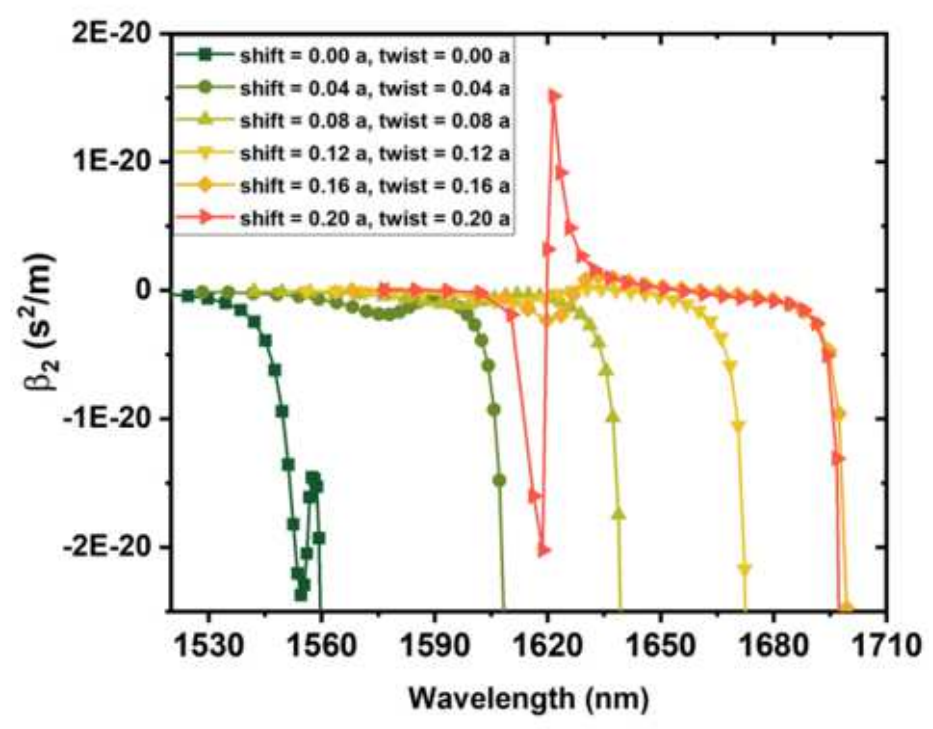

(b)

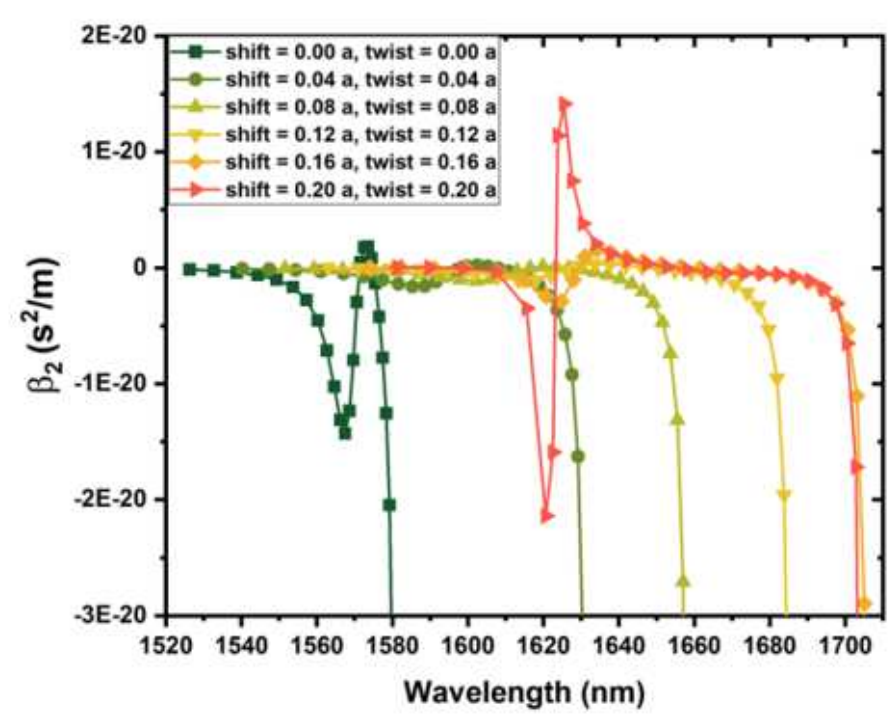

(d)

\section{Figure 15}

Dispersion characteristics corresponding to figure 14 (a-d). $\beta 2$ of PCW with holes and applied (a) shift and twist in one row (b) shift and twist in two rows. $\beta 2$ of PCW with rings and applied (c) shift and twist in one row (d) shift and twist in two rows. 


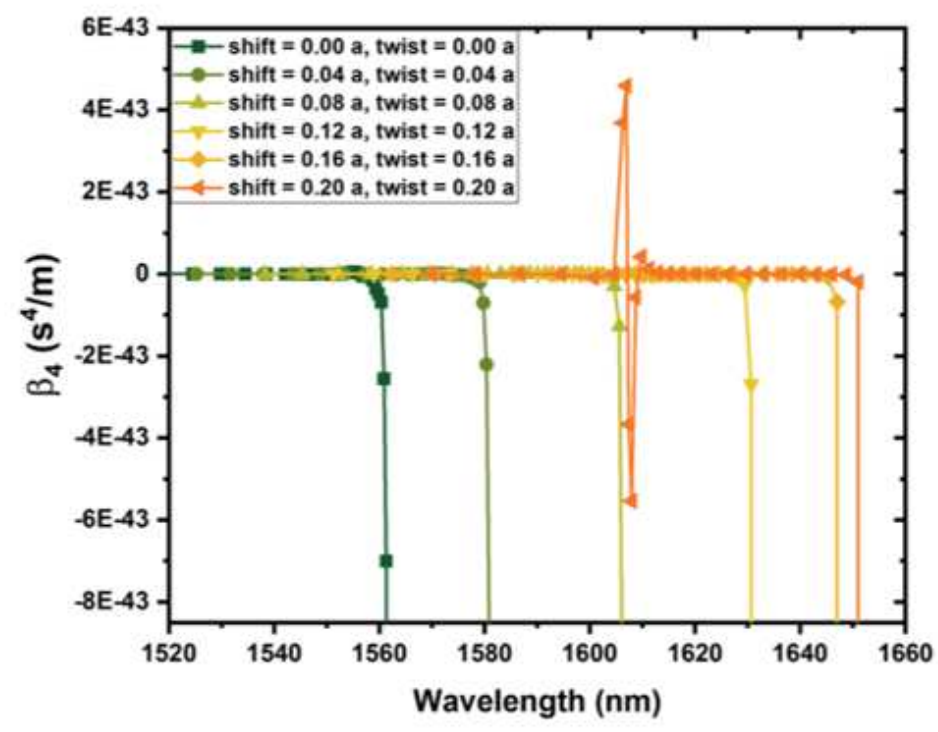

(a)

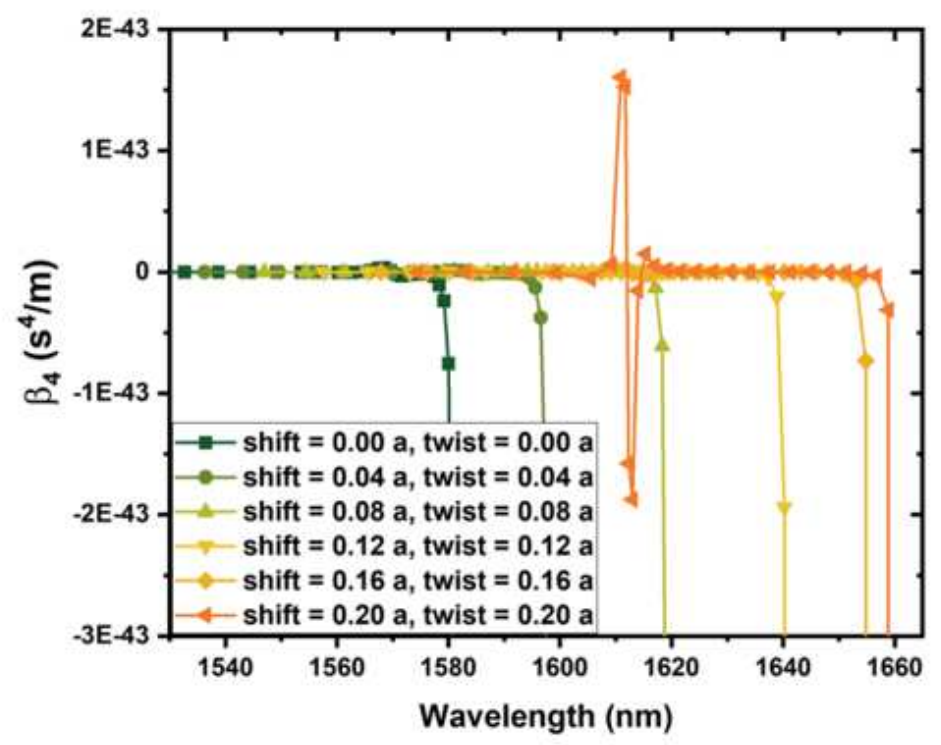

(c)

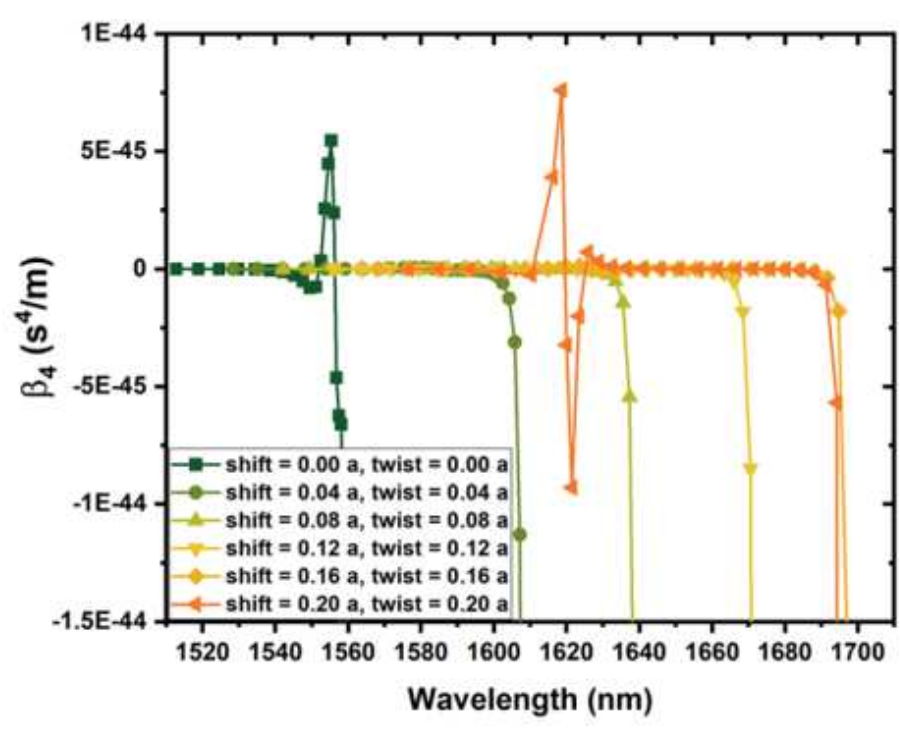

(b)

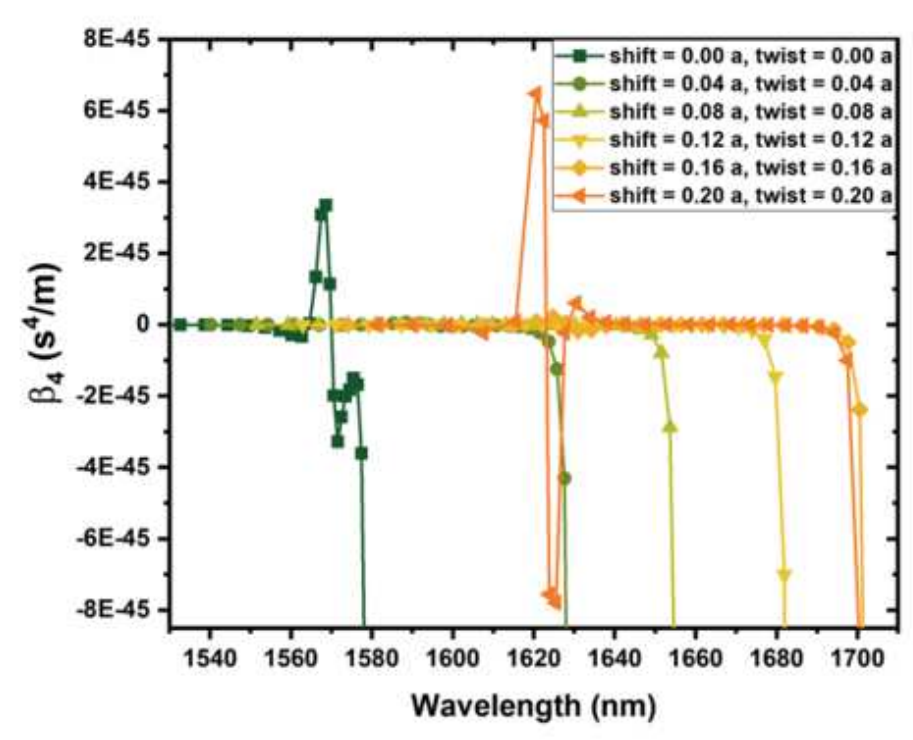

(d)

\section{Figure 16}

Dispersion characteristics corresponding to figure 14 (a-d). $\beta 4$ of PCW with holes and applied (a) shift and twist in one row (b) shift and twist in two rows. $\beta 4$ of PCW with rings and applied (c) shift and twist in one row (d) shift and twist in two rows. 